- This report was prepared as an account of work sponsored by an agency of the United States Government. Neither the United States Government nor any agency thereof, nor any of their employees, makes any warranty, express or implied, or assumes any legal liability or responsibility for the accuracy, completeness, or usefulness of any information, apparatus, product, or process disclosed, or represents that its use would not infringe privately owned rights. Reference herein to any specific commercial product, process, or service by trade name, trademark, manufacturer, or otherwise does not necessarily constitute or imply its endorsement, recommendation, or favoring by the United States Government or any agency thereof. The views and opinions of authors expressed herein do not necessarily state or reflect those of the United States Government or any agency thereof.

$$
\text { USGS-OFR- }-95-459
$$

Open-File Report 95-459

\title{
Results of a Seepage Investigation at Bear Creek Valley, Oak Ridge, Tennessee, January through September 1994
}

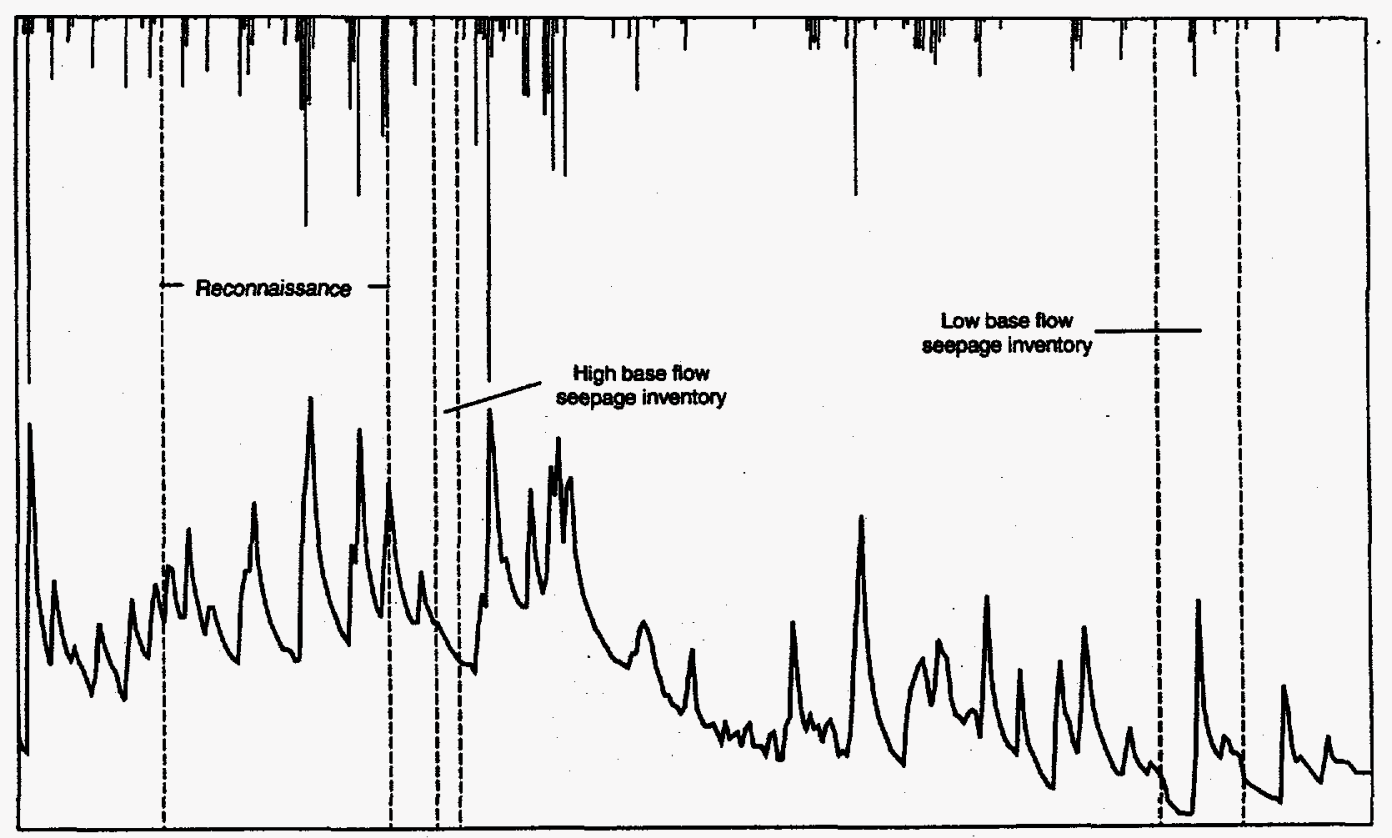

Prepared by the U.S. GEOLOGICAL SURVEY

in cooperation with the U.S. DEPARTMENT OF ENERGY

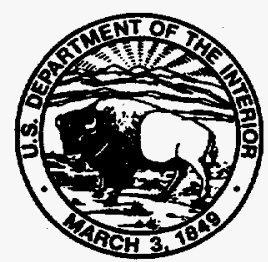


Cover illustration. See figure 2, page 5. 


\section{DISCLAMMER}

Portions of this document may be illegible in electronic image products. Images are produced from the best available original document. 


\section{Results of a Seepage Investigation at Bear Creek Valley, Oak Ridge, Tennessee, January through September 1994}

By JOHN A. ROBINSON and GREGORY C. JOHNSON

\section{U.S. GEOLOGICAL SURVEY}

Open-File Report 95-459

Prepared in cooperation with the

\section{U.S. DEPARTMENT OF ENERGY}

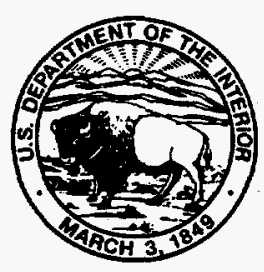

Nashville, Tennessee 


\title{
U.S. DEPARTMENT OF THE INTERIOR BRUCE BABBITT, Secretary
}

\author{
U.S. GEOLOGICAL SURVEY \\ Gordon P. Eaton, Director
}

Any use of trade, product, or firm name in this report is for identification purposes only and does not constitute endorsement by the U.S.

Geological Survey.

For additional information write to:

District Chief

U.S. Geological Survey

810 Broadway, Suite 500

Nashville, Tennessee 37203
Copies of this report may be purchased from:

U.S. Geological Survey

Branch of Information Services

Box 25286

Denver, Colorado 80225 


\section{CONTENTS}

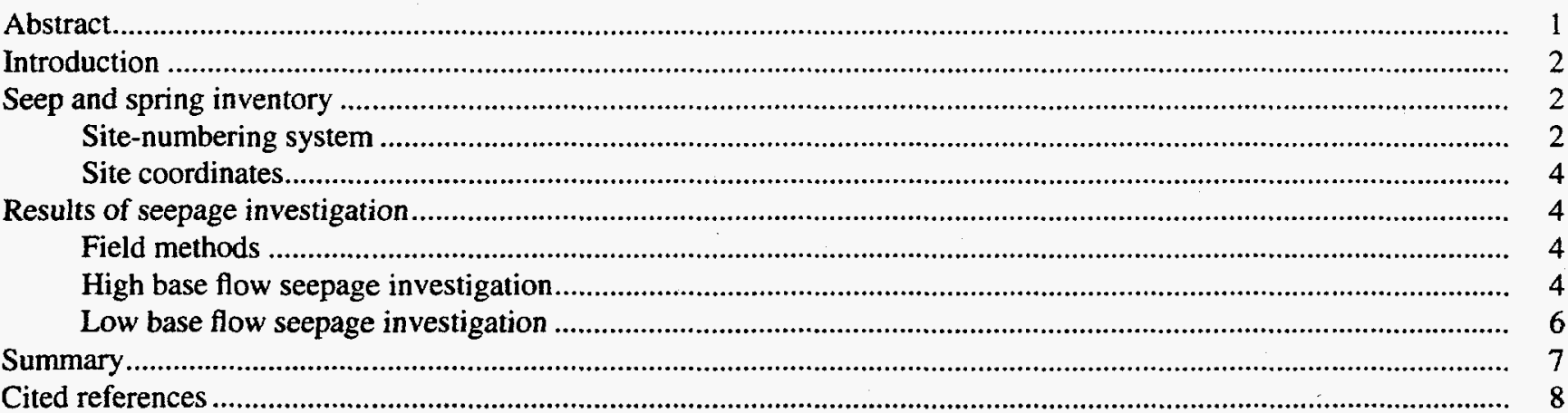

\section{PLÁTE}

[Plate is in two pieces in pocket]

1. Map showing location of seeps, springs, and stream-measurement sites for the seepage investigation at Bear Creek Valley, Oak Ridge, Tennessee, 1994

\section{FIGURES}

1. Map showing location of the study area in Bear Creek Valley, Oak Ridge, Tennessee

2. Graph showing mean daily discharge of Bear Creek near State Route 95, at Oak Ridge, Tennessee, and daily rainfall at Whiteoak Creek, at Oak Ridge, Tennessee, from

December 1, 1993 through October 31, 1994

\section{TABLES}

1. Size of sampling areas in the Bear Creek Valley seepage investigation, Oak Ridge, Tennessee

2. State plane coordinates for seeps, springs, wetlands, and stream-measurement sites at Bear Creek Valley, Oak Ridge, Tennessee

3. Discharge and water-quality data for the high base flow seepage investigation at Bear Creek Valley, Oak Ridge, Tennessee, March 14 through March 19, 1994

4. Statistical summary of discharge and water-quality data for the high base flow seepage investigation at Bear Creek Valley, Oak Ridge, Tennessee, March 14 through March 19, 1995.

5. Discharge and water-quality data for the low base flow seepage investigation at Bear Creek Valley, Oak Ridge, Tennessee, September 9 through September 29, 1994

6. Statistical summary of the discharge and water-quality data for the low base flow seepage investigation at Bear Creek Valley, Oak Ridge, Tennessee, September 9 through September 29, 1994 


\begin{tabular}{rll}
\hline Multiply & By & To Obtain \\
\hline cubic foot per second $\left(\mathrm{ft}^{3} / \mathrm{s}\right)$ & 0.02832 & cubic meter per second \\
foot $(\mathrm{ft})$ & 0.3048 & meter \\
acre & 0.4047 & square hectare \\
square mile $\left(\mathrm{mi}^{2}\right)$ & 2.590 & square kilometer \\
mile $(\mathrm{mi})$ & 1.609 & kilometer \\
microsiemens per centimeter & 1 & micromhos per \\
at $25^{\circ} \mathrm{C}(\mu \mathrm{S} / \mathrm{cm})$ & & centimeter at $25^{\circ} \mathrm{C}$ \\
\hline
\end{tabular}

Temperature in degrees Celsius $\left({ }^{\circ} \mathrm{C}\right)$ can be converted to degrees Fahrenheit $\left({ }^{\circ} \mathrm{F}\right)$ as follows:

$$
{ }^{\circ} \mathrm{F}=1.8 \times{ }^{\circ} \mathrm{C}+32
$$

Sea level: In this report "sea level" refers to the National Geodetic Vertical Datum of 1929-a geodetic datum derived from a general adjustment of first-order level nets of the United States and Canada, formally called Sea Level Datum of 1929. 


\title{
Results of a Seepage Investigation at Bear Creek Valley, Oak Ridge, Tennessee, January through September 1994
}

\author{
By John A. Robinson and Gregory C. Johnson
}

\section{ABSTRACT}

A seepage investigation was conducted of 4,600 acres of Bear Creek Valley southwest of the Y-12 Plant, Oak Ridge, Tennessee, for the period of January through September 1994. The data were collected to help the Y-12 Environmental Restoration Program develop a better understanding of ground-water and surface-water interactions, recharge and discharge relations, and ground-water flow patterns. The project was divided into three phases: a reconnaissance and mapping of seeps, springs, and stream-measurement sites; a high base flow seepage investigation; and a low base flow seepage investigation.

The reconnaissance was conducted from January 6 to March 1, 1994, to identify and map the locations of seeps, springs, and streammeasurement sites. A total of 701 sites were identified. They consisted of 382 stream-measurement sites, 265 seeps, 48 springs, and 6 wetlands. A global positioning system was used to locate 680 sites to within 3- to 5-meter accuracy.

The high base flow seepage investigation was conducted from March 14 through March 19, 1994. Measurements were made at 579 of the 701 sites identified in the reconnaissance that still had flowing water. Flow rates ranged from less than 0.005 to 6.89 cubic feet per second $\left(\mathrm{ft}^{3} / \mathrm{s}\right)$ for the streams, from less than 0.005 to $0.13 \mathrm{ft}^{3} / \mathrm{s}$ for the seeps, and from less than 0.005 to $1 \mathrm{ft}^{3} / \mathrm{s}$ for the springs. $\mathrm{pH}$ ranged from 5.0 to 8.4 for the streams, from 5.1 to 8.2 for the seeps, from 5.3 to 8.0 for the springs, and from 6.7 to 6.8 for the wetland sites. Specific conductance ranged from 16 to 1,670 microsiemens per centimeter $(\mu \mathrm{S} / \mathrm{cm})$ for the streams, from 17 to $1,710 \mu \mathrm{S} / \mathrm{cm}$ for the seeps, from 14 to $1,150 \mu \mathrm{S} / \mathrm{cm}$ for the springs, and from 102 to $160 \mu \mathrm{S} / \mathrm{cm}$ for the wetland sites. Temperature ranged from 4.5 to $16.0^{\circ} \mathrm{C}$ for the streams, from 5.0 to $21.0^{\circ} \mathrm{C}$ for the seeps, from 6.0 to $13.5^{\circ} \mathrm{C}$ for the springs, and from 13.0 to $19.5^{\circ} \mathrm{C}$ for the wetland sites. Dissolved oxygen ranged from 4.8 to 11.2 milligrams per liter (mg/L) for the streams, 1.2 to $11.3 \mathrm{mg} / \mathrm{L}$ for the seeps, and from 0.6 to $11.0 \mathrm{mg} / \mathrm{L}$ for the springs. Dissolved oxygen at a wetland site measured $3.8 \mathrm{mg} / \mathrm{L}$.

The low base flow investigation was conducted from September 9 through September 29, 1994. The stream sites, seeps, and springs that had flow during the high base flow seepage investigation were revisited. One-hundred seventy-six of the stream sites visited still had flow. Discharge ranged from less than 0.005 to $0.76 \mathrm{ft}^{3} / \mathrm{s} ; \mathrm{pH}$, from 4.8 to 8.3 ; specific conductance, from 47 to $2,030 \mu \mathrm{S} / \mathrm{cm}$; temperature, from 13.5 to $22.5^{\circ} \mathrm{C}$; and dissolved oxygen, from 3.6 to $8.7 \mathrm{mg} / \mathrm{L}$. Twenty-five of the seeps visited were flowing and had discharge ranging from less than 0.005 to $0.01 \mathrm{ft}^{3} / \mathrm{s} ; \mathrm{pH}$, from 6.0 to 7.7 ; specific conductance, from 36 to $395 \mu \mathrm{S} / \mathrm{cm}$; temperature, from 16.0 to $21.0^{\circ} \mathrm{C}$; and dissolved oxygen, from 2.2 to $9.0 \mathrm{mg} / \mathrm{L}$. Thirty springs visited were flowing and had discharge ranging from less than 0.005 to $0.37 \mathrm{ft}^{3} / \mathrm{s} ; \mathrm{pH}$, from 6.5 to 7.7 ; specific conductance, from 26 to $1,220 \mu \mathrm{S} / \mathrm{cm}$; temperature, from 14.0 to $20.0^{\circ} \mathrm{C}$; and dissolved oxygen, from 1.0 to $9.2 \mathrm{mg} / \mathrm{L}$. All of the wetland sites visited were dry. 


\section{INTRODUCTION}

The Oak Ridge Reservation (ORR) is located in East Tennessee in the western part of the Valley and Ridge Province. The 58,000-acre ORR is bounded on the northeast, southeast, and southwest by the Clinch River, and on the northwest by Blackoak Ridge (McMaster, 1967). The three major facilities within the ORR are Y-12, a research, development, and production center; X-10, the Oak Ridge National Laboratory (ORNL), a research and development center; and K-25, the Gaseous Diffusion Plant (ORGDP), a production center that was closed in 1986.

During 1994, the U.S. Geological Survey (USGS), in cooperation with the U.S. Department of Energy, conducted a seepage investigation of Bear Creek Valley, in which the Y-12 Plant is located. Information provided by this study will aid the Y-12 Environmental Restoration Program, Groundwater Operable Units Remedial Investigations Project develop a better understanding of ground-water and surface-water interactions on the ORR.

The study involved three phases of activity: (1) a reconnaissance to inventory and map seeps, springs, and stream-measurement sites; (2) the measurement of discharge and water-quality characteristics under high base flow conditions, and (3) the measurement of discharge and water-quality characteristics under low base flow conditions. This report describes the results of the investigation. It includes a map showing measurement site locations and tables that list the coordinates for each site and measurements of discharge, $\mathrm{pH}$, specific conductance, temperature, and dissolved oxygen.

The study area is bounded by Pine Ridge on the northwest, Chestnut Ridge on the southeast, the Clinch River on the southwest, and the Y-12 Plant on the northeast (fig. 1). Bear Creek Valley southwest of the Y-12 Plant is drained by two streams: Bear Creek, which exits through a water gap in Pine Ridge, and Grassy Creek, which discharges to the Clinch River. The 4,600 acres within the study area were divided into 22 sub-areas for measurement purposes (table 1). Certain locations within these areas were excluded from the study because of safety or security concerns.

\section{SEEP AND SPRING INVENTORY}

The reconnaissance was conducted from January 6 to March 1, 1994. For each of the 22 sampling
Table 1. Size of sampling areas in the Bear Creek Valley seepage investigation, Oak Ridge, Tennessee

\begin{tabular}{rcc}
\hline $\begin{array}{c}\text { Sampling } \\
\text { area }\end{array}$ & $\begin{array}{c}\text { Area } \\
\text { (acres) }\end{array}$ & $\begin{array}{c}\text { Area } \\
\text { (square miles) }\end{array}$ \\
\hline 1 & 303.3 & 0.47 \\
2 & 227.5 & 0.36 \\
3 & 239.6 & 0.37 \\
4 & 355.8 & 0.56 \\
5 & 171.0 & 0.27 \\
6 & 157.0 & 0.25 \\
7 & 191.7 & 0.30 \\
8 & 157.1 & 0.25 \\
9 & 124.8 & 0.19 \\
10 & 181.2 & 0.28 \\
11 & 154.2 & 0.24 \\
12 & 230.9 & 0.36 \\
13 & 233.6 & 0.36 \\
14 & 169.5 & 0.26 \\
15 & 224.2 & 0.35 \\
16 & 218.4 & 0.34 \\
17 & 216.8 & 0.34 \\
18 & 237.3 & 0.37 \\
19 & 239.8 & 0.37 \\
20 & 281.6 & 0.21 \\
21 & 133.1 & 0.21 \\
22 & 154.2 & 0.24 \\
\hline Total & $4,602.6$ & 7.19 \\
\hline
\end{tabular}

areas, all streams and tributaries were traversed to their source or to an area boundary. A total of 701 seeps, springs, and stream-measurement sites were staked and assigned unique identification numbers. The inventory consists of 265 seeps, 48 springs, 6 wetlands, and 382 stream-measurement sites (plate 1).

\section{Site-Numbering System}

Each site was assigned a unique identification number. The identification number consists of four or five digits beginning with the sampling area number followed by an upstream order number that ranges from 001 to 999 . This approach assigns the lowest number to the most downstream site in the basin and increasingly larger numbers to sites upstream. When a tributary is reached, site numbers increase along the tributary upstream to its source, and numbering resumes along the main channel.

The sites were classified as either a seep, spring, stream-measurement location, or wetland (tables 3 and 5, located at back of report). A seep was defined as a discharge of water at land surface without a distinguishable point outlet and generally at a low flow rate. A spring was defined as a discharge of water at a 


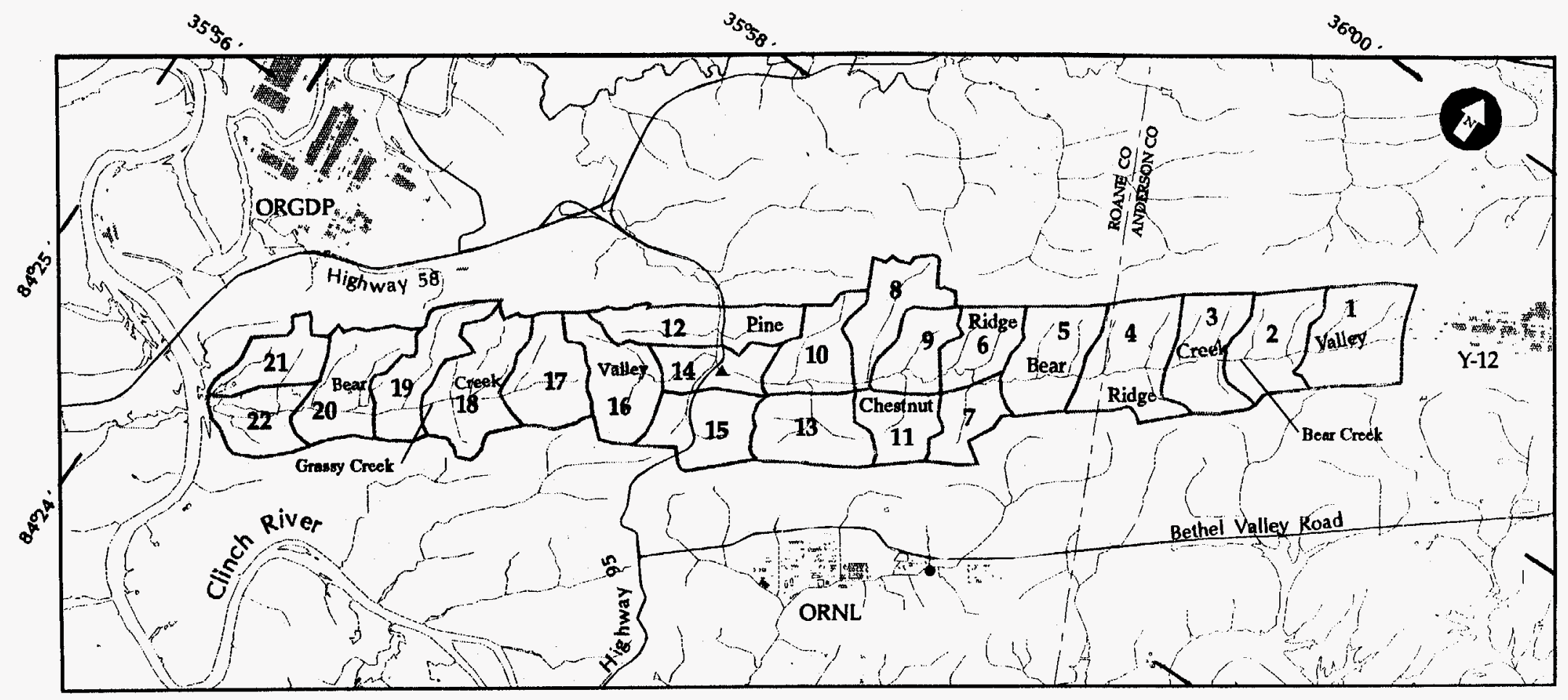

Baso from Tennessee Valley Authority S-16A Mep of the Oak Ridge, Tennessee Area, December 1987

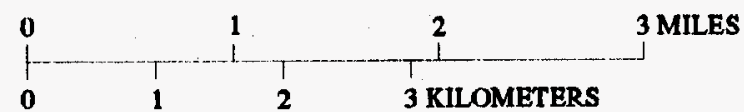

AREA BOUNDARY

TENNESSEE

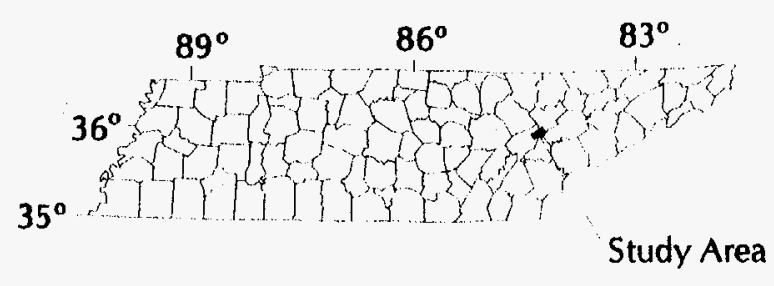

17 AREA NUMBER

- Stage gage

- RAIN GAGE

BUILDINGS

Figure 1. Study area in Bear Creek Valley, Oak Ridge, Tennessee. 
defined outlet or where water was upwelling. The presence of watercress usually was an indicator of a spring. Stream-measurement sites were located about every 500 feet along each channel with flowing water and at the confluence of tributaries with flow. A wetland was defined as a bog-like area with no outlet.

\section{Site Coordinates}

A global positioning system (GPS) was used to determine the coordinates of 680 sites to within 3- to 5-meter accuracy. The remaining sites were mapped by measuring their distances from the GPS points or other control points, plotting locations on field maps, and digitizing those points. Locations of the 701 sites inventoried are reported in state plane coordinates (table 2, located at back of report).

\section{RESULTS OF SEEPAGE INVESTIGATION}

Two seepage investigations were conducted, one during high base flow and one during low base flow. A waiting period of 72 hours after rainfall events was used to minimize the possible influence of ephemeral streams and springs (Mulholland, 1991). Measurements in each sampling area were completed in 1 day to minimize variations in flow rates. During the high base flow seepage investigation, discharge and water-quality measurements were made at each site that had flowing water. For the low base flow seepage investigation, only the seeps and springs that had flow during the high base flow investigation were revisited.

\section{Field Methods}

Four methods were used to measure discharge: (1) volumetric measurements, (2) current meter measurements, (3) surface velocity measurements, and (4) estimations.

Volumetric measurements are typically the most accurate method for quantifying discharge during low flow conditions. For this study, a volume of water was collected during a timed interval for four iterations, and the discharge was calculated as the average of the four measurements.

Pygmy-type current meters were used where practical. Using standard USGS current meter discharge measurement procedures, the accuracy of measurements range from about 5 percent error on some of . the larger streams to greater than 8 percent error on some sites with very low discharge.

The third type of discharge measurement involved the use of floats. The cross-sectional area of a stream was measured, and the average time for a float to travel a known distance for four iterations was determined. Discharge was then calculated and reported to $0.01 \mathrm{ft}^{3} / \mathrm{s}$.

The final, and least accurate, method was to estimate the discharge. This method was used in cases where the discharge was very small, and no other method was practical.

A notation was made to indicate sites where flow was visible, but less than the minimum reportable discharge. At sites where the flow was less than $0.005 \mathrm{ft}^{3} / \mathrm{s}$, the flow was reported as zero. For flows greater than $0.005 \mathrm{ft}^{3} / \mathrm{s}$, the discharge was rounded to the nearest hundredth of a cubic foot per second.

Field water-quality parameters consisted of specific conductance, temperature, $\mathrm{pH}$, and dissolved oxygen. The dissolved oxygen, $\mathrm{pH}$, and conductance meters used to collect these data were calibrated every morning before use, and again in the field if questionable values occurred. Calibration and use of the field meters were consistent with standard USGS procedures.

Discharge at the stream gaging station, at Bear Creek at Highway 95 near Oak Ridge, Tennessee (03538270), and rainfall at the gaging station, at Whiteoak Creek near Melton Valley Road (03536550), were used as indicators of the hydrologic conditions at the study area (fig. 2). During the reconnaissance, January 6 through March 1, 1994, mean daily discharge at Bear Creek ranged from 6.1 to $329 \mathrm{ft}^{3} / \mathrm{s}$. The mean daily discharge ranged from $5.8 \mathrm{ft}^{3} / \mathrm{s}$ to $11.7 \mathrm{ft}^{3} / \mathrm{s}$ during the high base flow seepage investigation and from 0.59 to $14 \mathrm{ft}^{3} / \mathrm{s}$ for the low base flow seepage investigation.

\section{High Base Flow Seepage Investigation}

The high base flow seepage investigation was conducted from March 14 through March 19, 1994. All 701 sites identified during the reconnaissance were revisited. Discharge and water-quality measurements were made at 579 of the 701 sites that still had flowing water (table 3, located at back of report). At 174 of the 579 sites with flowing water, the flow was reported as zero with a notation that flow was below the minimum reportable discharge $\left(0.005 \mathrm{ft}^{3} / \mathrm{s}\right)$. Flow rates ranged 
DISCHARGE, IN CUBIC FEET PER SECOND

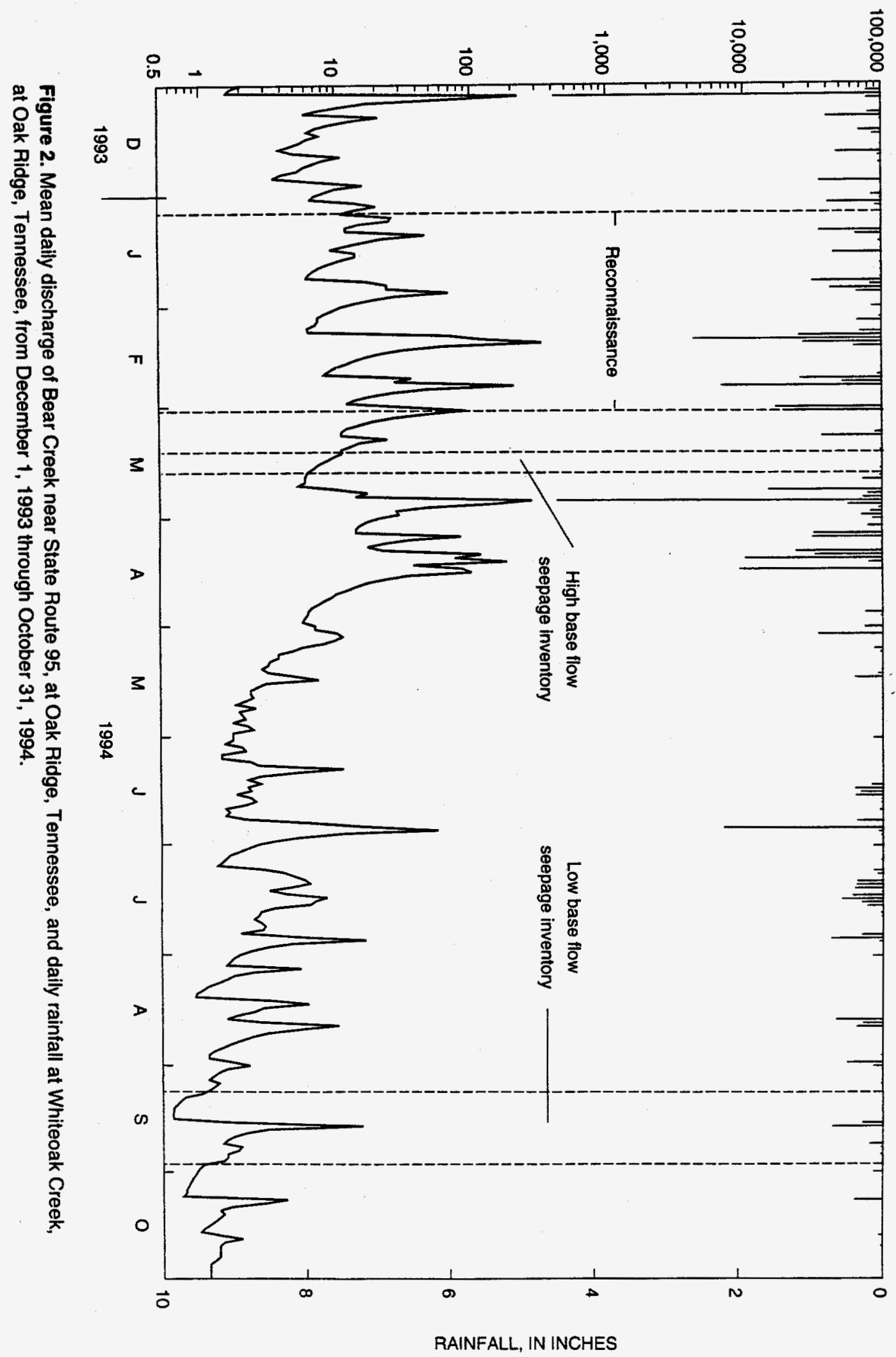


from 0 to $6.89 \mathrm{ft}^{3} / \mathrm{s}$ for the streams, from 0 to $0.13 \mathrm{ft}^{3} / \mathrm{s}$ for the seeps, and from below the reporting limit to $1 \mathrm{ft}^{3} / \mathrm{s}$ for the springs (table 4). $\mathrm{pH}$ values ranged from 5.0 to 8.4 for the streams, from 5.1 to 8.2 for the seeps, from 5.3 to 8.0 for the springs, and 6.7 to 6.8 for the wetlands. Specific conductance ranged from 16 to 1,670 microsiemens per centimeter $(\mu \mathrm{S} / \mathrm{cm})$ for the streams, from 17 to $1,710 \mu \mathrm{S} / \mathrm{cm}$ for the seeps, from 14 to $1,150 \mu \mathrm{S} / \mathrm{cm}$ for the springs, and from 102 to $160 \mu \mathrm{S} / \mathrm{cm}$ for the wetlands. Temperature ranged from 4.5 to $16.0^{\circ} \mathrm{C}$ for the streams, 5.0 to $21.0^{\circ} \mathrm{C}$ for the seeps, 6.0 to $13.5^{\circ} \mathrm{C}$ for the springs, and 13.0 to $19.5^{\circ} \mathrm{C}$ for the wetlands. Dissolved oxygen ranged from 4.8 to 11.2 milligrams per liter $(\mathrm{mg} / \mathrm{L})$ for the streams, from 1.2 to $11.3 \mathrm{mg} / \mathrm{L}$ for the seeps, and from 0.6 to $11.0 \mathrm{mg} / \mathrm{L}$ for the springs. Dissolved oxygen at a wetland site measured $3.8 \mathrm{mg} / \mathrm{L}$.

\section{Low Base Flow Seepage Investigation}

Streams, seeps, and springs that were flowing during the high base flow seepage investigation were

Table 4. Statistical summary of discharge and water-quality data for the high base flow seepage investigation at Bear Creek Valley, Oak Ridge, Tennessee, March 14 through March 19, 1995

$\left[\mathrm{ft}^{3} / \mathrm{s}\right.$, cubic foot per second; $\mu \mathrm{S} / \mathrm{cm}$, microsiemens per centimeter at 25 degrees Celsius; ${ }^{\circ} \mathrm{C}$, degrees Celsius; mg/L, milligrams per liter; --, no data]

\begin{tabular}{|c|c|c|c|c|c|}
\hline Statistic & $\begin{array}{l}\text { Flow } \\
\left(\mathrm{t}^{3} / \mathrm{s}\right)\end{array}$ & pH & $\begin{array}{c}\text { Specific } \\
\text { conductance } \\
(\mu \mathrm{S} / \mathrm{cm})\end{array}$ & $\begin{array}{c}\text { Temperature } \\
\left({ }^{\circ} \mathrm{C}\right) \\
\end{array}$ & $\begin{array}{c}\text { Dissolved oxygen } \\
\text { (mg/L) }\end{array}$ \\
\hline \multicolumn{6}{|c|}{382 Stream-measurement sites } \\
\hline Number of & & & & & \\
\hline $\begin{array}{l}\text { measurements. } \\
\text { Minimum value }\end{array}$ & 296 & 351 & 351 & 351 & 22 \\
\hline Minimum value & 0 & 5.0 & 16 & 4.5 & 4.8 \\
\hline Maximum value & 6.89 & 8.4 & 1,670 & 16 & 11.2 \\
\hline Mean & 0.53 & -- & 162 & 10.2 & 9.0 \\
\hline Standard deviation & 1.3 & - & 209 & 2.3 & 1.7 \\
\hline Median & .06 & 7.3 & 90 & 10 & 9.2 \\
\hline \multicolumn{6}{|c|}{265 Seep sites } \\
\hline $\begin{array}{l}\text { Number of } \\
\text { measurements. }\end{array}$ & 58 & 166 & 169 & 169 & 130 \\
\hline Minimum value & 0 & 5.1 & 17 & 5 & 1.2 \\
\hline Maximum value & .13 & 8.2 & 1,710 & 21 & 11.3 \\
\hline Mean & .01 & -- & 127 & 10.5 & 6.7 \\
\hline Standard deviation & .02 & -- & 176 & 2.5 & 2.5 \\
\hline Median & .01 & 6.7 & 66 & 10 & 7.0 \\
\hline \multicolumn{6}{|c|}{48 Spring sites } \\
\hline $\begin{array}{l}\text { Number of } \\
\text { measurements. }\end{array}$ & 35 & 47 & 47 & 47 & 47 \\
\hline Minimum value & 0 & 5.3 & 14 & 6 & .6 \\
\hline Maximum value & 1 & 8.0 & 1,150 & 13.5 & 11.0 \\
\hline Mean & .12 & -- & 214 & 11.5 & 8.3 \\
\hline Standard deviation & .21 & -- & 255 & 1.5 & 2.4 \\
\hline Median & .03 & 7.1 & 118 & 12 & 9.0 \\
\hline \multicolumn{6}{|c|}{6 Wetland sites } \\
\hline $\begin{array}{l}\text { Number of } \\
\text { measurements. }\end{array}$ & 0 & 2 & 2 & 2 & 1 \\
\hline Minimum value & - & 6.7 & 102 & 13 & 3.8 \\
\hline Maximum value & -- & 6.8 & 160 & 19.5 & 3.8 \\
\hline Mean & -- & -- & 131 & 16.3 & 3.8 \\
\hline Standard deviation & -- & -- & - & -- & -- \\
\hline Median & -- & -- & - & - & - \\
\hline
\end{tabular}

6 Results of a Seepage Investigation at Bear Creek Valley, Oak Ridge, Tennessee, January through September 1994 
revisited during September 9 through September 29 , 1994, to make low base flow measurements (table 5 , located at back of report). At 75 stream sites, 23 spring sites, and 22 seep sites, the flow rate was reported as zero with a notation that flow was below the minimum reportable discharge. Of the 382 stream sites visited, 176 had flow. Discharge ranged from 0 to $0.76 \mathrm{ft}^{3} / \mathrm{s} ; \mathrm{pH}$, from 4.8 to 8.3 ; specific conductance, from 47 to $2,030 \mu \mathrm{S} / \mathrm{cm}$; temperature, from 13.5 to $22.5^{\circ} \mathrm{C}$; and dissolved oxygen, from 3.6 to $8.7 \mathrm{mg} / \mathrm{L}$. Twenty-five of the 147 seeps visited were flowing and had discharge ranging from 0 to $0.01 \mathrm{ft}^{3} / \mathrm{s} ; \mathrm{pH}$, from 6.0 to 7.7 ; specific conductance, from 36 to $395 \mu \mathrm{S} / \mathrm{cm}$; temperature, from 16.0 to $21.0^{\circ} \mathrm{C}$; and dissolved oxygen, from 2.2 to $9.0 \mathrm{mg} / \mathrm{L}$. Thirty of the 48 springs visited were flowing and had discharge ranging from 0 to $0.37 \mathrm{ft}^{3} / \mathrm{s} ; \mathrm{pH}$, from 6.5 to 7.7 ; specific conductance, from 26 to $1,220 \mu \mathrm{S} / \mathrm{cm}$; temperature, from 14.0 to $20.0^{\circ} \mathrm{C}$; and dissolved oxygen, from 1.0 to $9.2 \mathrm{mg} / \mathrm{L}$ (table 6 ). All of the wetland sites visited were dry.

\section{SUMMARY}

A seepage study was made of 4,600 acres in Bear Creek Valley near the Y-12 Plant on the Oak Ridge Reservation. The study was conducted in three phases: a reconnaissance and mapping of the sites, a high base flow seepage investigation, and a low base flow seepage investigation. The reconnaissance was conducted from January 6 to March 1, 1994, to identify and map seeps, springs, and stream-measurement sites. A total of 701 sites were identified, which consisted of 265 seeps, 48 springs, 6 wetlands, and 382 stream-measurement sites.

The high base flow seepage investigation was conducted from March 14 through March 19, 1994.

Table 6. Statistical summary of the discharge and water-quality data for the low base flow seepage investigation at Bear Creek Valley, Oak Ridge, Tennessee, September 9 through September 29, 1994

$\left[\mathrm{fr}^{3} / \mathrm{s}\right.$, cubic foot per second; $\mu \mathrm{S} / \mathrm{cm}$, microsiemens per centimeter at 25 degrees Celsius; ${ }^{\circ} \mathrm{C}$, degrees Celsius; mg/L, milligrams per liter; --, no data]

\begin{tabular}{llcccc}
\hline Statistic & Flow & pH & $\begin{array}{c}\text { Speclfic } \\
\text { conductance } \\
(\mu \mathrm{s} / \mathrm{cm})\end{array}$ & $\begin{array}{c}\text { Temperature } \\
\left.{ }^{\circ} \mathrm{C}\right)\end{array}$ & $\begin{array}{c}\text { Dissolved oxygen } \\
(\mathrm{mg} / \mathrm{L})\end{array}$ \\
\hline
\end{tabular}

Number of measurements.

Minimum value

Maximum value

Mean

Standard deviation

Median

Number of measurements.

Minimum value

Maximum value

Mean

Standard deviation

Median

Number of measurements.

Minimum value

Maximum value

Mean

Standard deviation

Median

\section{Stream-measurement sites}

$\begin{array}{cc}101 & 175 \\ 0 & 4.8 \\ 0.76 & 8.3 \\ .1 & -- \\ .16 & -- \\ .02 & 7.7\end{array}$

175
47
2,030
381
350
275

174

13.5

22.5

18.4

1.6

18.5
13

3.6

8.7

6.9

1.7

7.8
265 Seep sites
25

6.0

7.7

$-$

$-$

7.2

25
36
395
172
114
132

25

16

21

18.5

1

18.5

48 Spring sites
30

6.5

7.7

$-$

$-$

7.1

\begin{tabular}{cc}
25 & 26 \\
14 & 1.0 \\
20 & 9.2 \\
16.5 & 7.0 \\
2 & 1.9 \\
17 & 7.7 \\
\hline
\end{tabular}

13

2.2

9.0

6.7

2.2

7.7

\section{Spring sites}

$\begin{array}{ll}0 & 6.5 \\ 0.37 & 7.7 \\ .1 & - \\ .12 & - \\ .02 & 7.1\end{array}$

9.2 
Measurements were made at 579 of the 701 sites identified in the reconnaissance that still had flowing water. At 174 of the 579 sites, flow was insufficient to measure and was reported as zero with a notation that flow was below the minimum reportable discharge $\left(0.005 \mathrm{ft}^{3} / \mathrm{s}\right)$. Flow rates ranged from 0 to $6.89 \mathrm{ft}^{3} / \mathrm{s}$ for the streams, from 0 to $0.13 \mathrm{ft}^{3} / \mathrm{s}$ for the seeps, and from 0 to $1 \mathrm{ft}^{3} / \mathrm{s}$ for the springs. $\mathrm{pH}$ values ranged from 5.0 to 8.4 for the streams, from 5.1 to 8.2 for the seeps, from 5.3 to 8.0 for the springs, and 6.7 to 6.8 for the wetlands. Specific conductance ranged from 16 to $1,670 \mu \mathrm{S} / \mathrm{cm}$ for the streams, from 17 to $1,710 \mu \mathrm{S} / \mathrm{cm}$ for the seeps, from 14 to $1,150 \mu \mathrm{S} / \mathrm{cm}$ for the springs, and from 102 to $160 \mu \mathrm{S} / \mathrm{cm}$ for the wetlands. Temperature ranged from 4.5 to $16.0^{\circ} \mathrm{C}$ for the streams, 5.0 to $21.0^{\circ} \mathrm{C}$ for the seeps, 6.0 to $13.5^{\circ} \mathrm{C}$ for the springs, and 13.0 to $19.5^{\circ} \mathrm{C}$ for the wetlands. Dissolved oxygen ranged from 4.8 to 11.2 milligrams per liter $(\mathrm{mg} / \mathrm{L})$ for the streams, 1.2 to $11.3 \mathrm{mg} / \mathrm{L}$ for the seeps, and from 0.6 to $11.0 \mathrm{mg} / \mathrm{L}$ for the springs. Dissolved oxygen at a wetland site measured $3.8 \mathrm{mg} / \mathrm{L}$.

The low base flow investigation was conducted from September 9 through September 29, 1994. The streams, seeps, and springs that had flow during the high base flow seepage investigation were revisited. At 75 stream sites, 23 springs, and 22 seeps, the flow rate was reported as zero with a notation that flow was below the minimum reportable discharge. Of the $\mathbf{3 8 2}$ stream sites visited, 176 had flow. Discharge ranged from below the reporting limit to $0.76 \mathrm{ft}^{3} / \mathrm{s} ; \mathrm{pH}$, from 4.8 to 8.3 ; specific conductance, from 47 to $2,030 \mu \mathrm{S} / \mathrm{cm}$; temperature, from 13.5 to $22.5^{\circ} \mathrm{C}$; and dissolved oxygen, from 3.6 to $8.7 \mathrm{mg} / \mathrm{L}$. Twenty-five of the 147 seeps visited were flowing and had discharge ranging from 0 to $0.01 \mathrm{ft}^{3} / \mathrm{s} ; \mathrm{pH}$, from 6.0 to 7.7 ; specific conductance, from 36 to $395 \mu \mathrm{S} / \mathrm{cm}$; temperature, from 16.0 to $21.0^{\circ} \mathrm{C}$; and dissolved oxygen, from 2.2 to $9.0 \mathrm{mg} / \mathrm{L}$. Thirty of the 48 springs visited were flowing and had discharge ranging from 0 to $0.37 \mathrm{ft}^{3} / \mathrm{s}$; $\mathrm{pH}$, from 6.5 to 7.7 ; specific conductance, from 26 to $1,220 \mu \mathrm{S} / \mathrm{cm}$; temperature, from 14.0 to $20.0^{\circ} \mathrm{C}$; and dissolved oxygen, from 1.0 to $9.2 \mathrm{mg} / \mathrm{L}$ (table 6). All of the wetland sites visited were dry.

\section{CITED REFERENCES}

McMaster, W.M., 1967, Hydrologic data for the Oak Ridge area Tennessee: U.S. Geological Survey Water-Supply Paper 1839-N, $90 \mathrm{p}$.

Mulholland, P.J., 1991, Effects of flowpath variation on the hydrogeochemical response of Walker Branch watershed to storms, in Sale, M.J., and Presley, P.M. compilers, Extended abstracts from Fourth Tennessee Water Resources Symposium, Knoxville, Tenn., 1991: Tennessee Section of the American Water Resources Association, p. 63-67. 
Table 2. State plane coordinates for seeps, springs, wetlands, and stream-measurement sites at Bear Creek Valley, Oak Ridge, Tennessee

[N, north; W, west; GPS, global positioning system; DIG, digitized]

\begin{tabular}{|c|c|c|c|c|c|c|c|}
\hline \multirow{3}{*}{$\begin{array}{c}\begin{array}{c}\text { Site } \\
\text { number }\end{array} \\
1005\end{array}$} & \multicolumn{6}{|c|}{ State plane coordinates } & \multirow{3}{*}{$\begin{array}{c}\text { Method } \\
\text { GPS }\end{array}$} \\
\hline & \multicolumn{3}{|c|}{$\begin{array}{l}\text { Latitude }(\mathbf{N}) \\
\text { (degrees, minutes, } \\
\text { seconds) }\end{array}$} & \multicolumn{3}{|c|}{$\begin{array}{c}\text { Longitude (W) } \\
\text { (degrees, minutes, } \\
\text { seconds) }\end{array}$} & \\
\hline & 35 & 58 & 17.292 & -84 & 16 & 48.454 & \\
\hline 1010 & 35 & 58 & 17.566 & -84 & 16 & 48.223 & GPS \\
\hline 1015 & 35 & 58 & 20.687 & -84 & 16 & 47.831 & GPS \\
\hline 1020 & 35 & 58 & 22.904 & -84 & 16 & 47.662 & GPS \\
\hline 1025 & 35 & 58 & 25.129 & -84 & 16 & 51.064 & GPS \\
\hline 1030 & 35 & 58 & 24.197 & -84 & 16 & 49.652 & GPS \\
\hline 1035 & 35 & 58 & 27.358 & -84 & 16 & 51.488 & GPS \\
\hline 1040 & 35 & 58 & 27.394 & -84 & 16 & 51.769 & GPS \\
\hline 1045 & 35 & 58 & 27.293 & -84 & 16 & 51.258 & GPS \\
\hline 1050 & 35 & 58 & 25.277 & -84 & 16 & 47.183 & GPS \\
\hline 1055 & 35 & 58 & 26.530 & -84 & 16 & 46.006 & GPS \\
\hline 1060 & 35 & 58 & 27.444 & -84 & 16 & 45.325 & GPS \\
\hline 1065 & 35 & 58 & 28.402 & -84 & 16 & 43.342 & GPS \\
\hline 1070 & 35 & 58 & 28.592 & -84 & 16 & 45.325 & GPS \\
\hline 1075 & 35 & 58 & 29.060 & -84 & 16 & 47.050 & GPS \\
\hline 1080 & 35 & 58 & 29.629 & .84 & 16 & 46.798 & GPS \\
\hline 1085 & 35 & 58 & 30.850 & -84 & 16 & 46.682 & GPS \\
\hline 1090 & 35 & 58 & 32.628 & -84 & 16 & 48.788 & GPS \\
\hline 1095 & 35 & 58 & 32.999 & -84 & 16 & 49.008 & GPS \\
\hline 1100 & 35 & 58 & 34.136 & -84 & 16 & 49.548 & GPS \\
\hline 1105 & 35 & 58 & 35.260 & -84 & 16 & 46.276 & GPS \\
\hline 1110 & 35 & 58 & 36.358 & -84 & 16 & 46.560 & GPS \\
\hline 1115 & 35 & 58 & 37.553 & -84 & 16 & 43.666 & GPS \\
\hline 1120 & 35 & 58 & 36.044 & -84 & 16 & 46.744 & GPS \\
\hline 1125 & 35 & 58 & 36.408 & -84 & 16 & 46.978 & GPS \\
\hline 1130 & 35 & 58 & 39.475 & -84 & 16 & 45.106 & GPS \\
\hline 1135 & 35 & 58 & 44.090 & -84 & 16 & 49.786 & GPS \\
\hline 1140 & 35 & 58 & 40.051 & -84 & 16 & 45.307 & GPS \\
\hline 1145 & 35 & 58 & 42.064 & -84 & 16 & 42.701 & GPS \\
\hline 1148 & 35 & 58 & 45.210 & -84 & 16 & 43.979 & GPS \\
\hline 1150 & 35 & 58 & 47.514 & -84 & 16 & 45.134 & GPS \\
\hline 1155 & 35 & 58 & 42.276 & -84 & 16 & 42.942 & GPS \\
\hline 1480 & 35 & 58 & 17.083 & -84 & 16 & 47.438 & GPS \\
\hline 1485 & 35 & 58 & 19.038 & -84 & 16 & 44.033 & GPS \\
\hline 1490 & 35 & 58 & 22.102 & -84 & 16 & 41.362 & GPS \\
\hline 1495 & 35 & 58 & 22.976 & -84 & 16 & 40.559 & GPS \\
\hline 1500 & 35 & 58 & 26.130 & -84 & 16 & 36.934 & GPS \\
\hline 1502 & 35 & 58 & 25.028 & -84 & 16 & 35.375 & GPS \\
\hline 1505 & 35 & 58 & 29.208 & -84 & 16 & 35.278 & GPS \\
\hline 1510 & 35 & 58 & 29.060 & -84 & 16 & 36.433 & GPS \\
\hline
\end{tabular}

\begin{tabular}{|c|c|c|c|c|c|c|c|}
\hline \multirow{3}{*}{$\begin{array}{c}\begin{array}{c}\text { Site } \\
\text { number }\end{array} \\
1515\end{array}$} & \multicolumn{6}{|c|}{ State plane coordinates } & \multirow{3}{*}{$\begin{array}{c}\text { Method } \\
\text { GPS }\end{array}$} \\
\hline & \multicolumn{3}{|c|}{$\begin{array}{l}\text { Latitude }(\mathrm{N}) \\
\text { (degrees, minutes, } \\
\text { seconds) }\end{array}$} & \multicolumn{3}{|c|}{$\begin{array}{l}\text { Longitude (W) } \\
\text { (degrees, minutes, } \\
\text { seconds) }\end{array}$} & \\
\hline & 35 & 58 & 33.366 & -84 & 16 & 34.507 & \\
\hline 1520 & 35 & 58 & 34.982 & -84 & 16 & 33.496 & GPS \\
\hline 1525 & 35 & 58 & 38.096 & -84 & 16 & 31.422 & GPS \\
\hline 1530 & 35 & 58 & 39.580 & -84 & 16 & 30.626 & GPS \\
\hline 1535 & 35 & 58 & 36.620 & -84 & 16 & 34.122 & GPS \\
\hline 1540 & 35 & 58 & 37.859 & -84 & 16 & 33.784 & GPS \\
\hline 1545 & 35 & 58 & 38.294 & -84 & 16 & 33.416 & GPS \\
\hline 1550 & 35 & 58 & 42.791 & -84 & 16 & 31.361 & GPS \\
\hline 1555 & 35 & 58 & 43.432 & -84 & 16 & 31.598 & GPS \\
\hline 1560 & 35 & 58 & 44.929 & -84 & 16 & 33.154 & GPS \\
\hline 1565 & 35 & 58 & 44.609 & -84 & 16 & 33.892 & GPS \\
\hline 1570 & 35 & 58 & 45.577 & -84 & 16 & 35.551 & GPS \\
\hline 1573 & 35 & 58 & 48.947 & -84 & 16 & 38.057 & GPS \\
\hline 1575 & 35 & 58 & 49.811 & -84 & 16 & 38.323 & GPS \\
\hline 1576 & 35 & 58 & 43.288 & -84 & 16 & 30.896 & GPS \\
\hline 1577 & 35 & 58 & 44.814 & -84 & 16 & 30.749 & GPS \\
\hline 1579 & 35 & 58 & 47.338 & -84 & 16 & 30.936 & GPS \\
\hline 1582 & 35 & 58 & 52.871 & -84 & 16 & 29.694 & GPS \\
\hline 1585 & 35 & 58 & 55.052 & -84 & 16 & 30.860 & GPS \\
\hline 1600 & 35 & 58 & .28 .870 & -84 & 16 & 32.063 & GPS \\
\hline 1610 & 35 & 58 & 32.250 & -84 & 16 & 21.691 & GPS \\
\hline 2005 & 35 & 57 & 58.986 & -84 & 17 & 15.904 & GPS \\
\hline 2006 & 35 & 58 & 2.582 & -84 & 17 & 19.360 & GPS \\
\hline 2010 & 35 & 58 & 3.090 & -84 & 17 & 19.446 & GPS \\
\hline 2015 & 35 & 58 & 0.340 & -84 & 17 & 13.520 & GPS \\
\hline 2020 & 35 & 58 & 0.973 & -84 & 17 & 13.142 & GPS \\
\hline 2025 & 35 & 58 & 6.017 & -84 & 17 & 11.742 & GPS \\
\hline 2027 & 35 & 58 & 8.540 & -84 & 17 & 9.265 & GPS \\
\hline 2030 & 35 & 58 & 7.115 & -84 & 17 & 12.566 & GPS \\
\hline 2035 & 35 & 58 & 7.266 & -84 & 17 & 17.722 & DIG \\
\hline 2040 & 35 & 58 & 8.843 & -84 & 17 & 11.699 & GPS \\
\hline 2050 & 35 & 58 & 9.005 & -84 & 17 & 15.227 & GPS \\
\hline 2055 & 35 & 58 & 10.535 & -84 & 17 & 16.861 & GPS \\
\hline 2065 & 35 & 58 & 9.991 & -84 & 17 & 13.924 & GPS \\
\hline 2067 & 35 & 58 & 13.620 & -84 & 17 & 11.634 & GPS \\
\hline 2068 & 35 & 58 & 13.156 & -84 & 17 & 12.620 & GPS \\
\hline 2070 & 35 & 58 & 17.612 & -84 & 17 & 12.916 & GPS \\
\hline 2080 & 35 & 58 & 21.583 & -84 & 17 & 12.131 & GPS \\
\hline 2083 & 35 & 58 & 21.058 & -84 & 17 & 13.423 & GPS \\
\hline 2085 & 35 & 58 & 25.993 & -84 & 17 & 14.132 & GPS \\
\hline
\end{tabular}


Table 2. State plane coordinates for seeps, springs, wetlands, and stream-measurement sites at Bear Creek Valley,

Oak Ridge, Tennessee-Continued

\begin{tabular}{|c|c|c|c|c|c|c|c|}
\hline \multirow{3}{*}{$\begin{array}{c}\begin{array}{c}\text { Site } \\
\text { number }\end{array} \\
2090\end{array}$} & \multicolumn{6}{|c|}{ State plane coordinates } & \multirow{3}{*}{$\begin{array}{c}\text { Method } \\
\text { GPS }\end{array}$} \\
\hline & \multicolumn{3}{|c|}{$\begin{array}{l}\text { Latitude (N) } \\
\text { (degrees, minutes, } \\
\text { seconds) }\end{array}$} & \multicolumn{3}{|c|}{$\begin{array}{l}\text { Longitude (W) } \\
\text { (degrees, minutes, } \\
\text { seconds) }\end{array}$} & \\
\hline & 35 & 58 & 15.344 & -84 & 17 & 12.689 & \\
\hline 2095 & 35 & 58 & 18.224 & -84 & 17 & 8.822 & GPS \\
\hline 2100 & 35 & 58 & 11.932 & -84 & 17 & 16.919 & GPS \\
\hline 2110 & 35 & 58 & 15.956 & -84 & 17 & 19.482 & GPS \\
\hline 2120 & 35 & 58 & 16.270 & -84 & 17 & 19.313 & GPS \\
\hline 2125 & 35 & 58 & 12.734 & -84 & 17 & 15.389 & GPS \\
\hline 2130 & 35 & 58 & 17.414 & -84 & 17 & 15.576 & GPS \\
\hline 2135 & 35 & 58 & 17.494 & -84 & 17 & 15.864 & GPS \\
\hline 2140 & 35 & 58 & 20.150 & -84 & 17 & 15.929 & GPS \\
\hline 2145 & 35 & 58 & 25.687 & -84 & 17 & 16.217 & GPS \\
\hline 2150 & 35 & 58 & 27.394 & -84 & 17 & 17.070 & GPS \\
\hline 2155 & 35 & 58 & 21.706 & -84 & 17 & 18.589 & GPS \\
\hline 2160 & 35 & 58 & 25.464 & -84 & 17 & 19.280 & GPS \\
\hline 2170 & 35 & 58 & 0.520 & -84 & 17 & 7.462 & GPS \\
\hline 2175 & 35 & 57 & 57.683 & -84 & 17 & 3.768 & GPS \\
\hline 2180 & 35 & 57 & 56.945 & -84 & 17 & 2.040 & GPS \\
\hline 2185 & 35 & 58 & 3.367 & -84 & 17 & 6.457 & GPS \\
\hline 2190 & 35 & 58 & 6.712 & -84 & 17 & 1.417 & GPS \\
\hline 2195 & 35 & 58 & 8.890 & -84 & 16 & 58.231 & GPS \\
\hline 2200 & 35 & 58 & $\begin{array}{r}11.762 \\
-\end{array}$ & -84 & 16 & 58.710 & GPS \\
\hline 2205 & 35 & 58 & 13.242 & -84 & 16 & 58.750 & GPS \\
\hline 2210 & 35 & 58 & 13.444 & -84 & 17 & 0.211 & GPS \\
\hline 2225 & 35 & 58 & 18.260 & -84 & 17 & 2.508 & GPS \\
\hline 2230 & 35 & 58 & 22.699 & -84 & 17 & 4.650 & GPS \\
\hline 2235 & 35 & 58 & 24.186 & -84 & 17 & 7.184 & GPS \\
\hline 2240 & 35 & 58 & 25.493 & -84 & 17 & 7.649 & GPS \\
\hline 2245 & 35 & 58 & 28.870 & -84 & 17 & 10.342 & GPS \\
\hline 2260 & 35 & 58 & 30.389 & -84 & 17 & 10.860 & GPS \\
\hline 2270 & 35 & 58 & 27.044 & -84 & 17 & 8.113 & GPS \\
\hline 2275 & 35 & 58 & 23.246 & -84 & 17 & 2.450 & GPS \\
\hline 2280 & 35 & 58 & 26.522 & -84 & 17 & 4.603 & GPS \\
\hline 2285 & 35 & 58 & 24.931 & -84 & 17 & 1.313 & GPS \\
\hline 2290 & 35 & 58 & 33.762 & -84 & 16 & 58.940 & GPS \\
\hline 2295 & 35 & 58 & 33.895 & -84 & 16 & 58.735 & GPS \\
\hline 2310 & 35 & 58 & 36.592 & -84 & 17 & 0.211 & GPS \\
\hline 2315 & 35 & 58 & 9.487 & -84 & 16 & 56.536 & GPS \\
\hline 2320 & 35 & 58 & 9.570 & -84 & 16 & 56.381 & GPS \\
\hline 2325 & 35 & 58 & 7.763 & -84 & 16 & 54.952 & GPS \\
\hline 2330 & 35 & 58 & 7.547 & -84 & 16 & 53.638 & GPS \\
\hline 2335 & 35 & 58 & 10.240 & -84 & 16 & 55.096 & GPS \\
\hline
\end{tabular}

\begin{tabular}{|c|c|c|c|c|c|c|c|}
\hline \multirow{3}{*}{$\begin{array}{c}\begin{array}{c}\text { Site } \\
\text { number }\end{array} \\
2337\end{array}$} & \multicolumn{6}{|c|}{ State plane coordinates } & \multirow{3}{*}{$\begin{array}{c}\text { Method } \\
\text { GPS }\end{array}$} \\
\hline & \multicolumn{3}{|c|}{$\begin{array}{l}\text { Latitude }(\mathrm{N}) \\
\text { (degrees, minutes, } \\
\text { seconds) }\end{array}$} & \multicolumn{3}{|c|}{$\begin{array}{l}\text { Longitude (W) } \\
\text { (degrees, minutes, } \\
\text { seconds) }\end{array}$} & \\
\hline & 35 & 58 & 8.987 & -84 & 16 & 53.346 & \\
\hline 2339 & 35 & 58 & 7.543 & -84 & 16 & 52.741 & GPS \\
\hline 2341 & 35 & 58 & 9.196 & -84 & 16 & 52.626 & GPS \\
\hline 2345 & 35 & 58 & 14.491 & -84 & 16 & 53.058 & GPS \\
\hline 3005 & 35 & 57 & 39.964 & -84 & 17 & 43.339 & GPS \\
\hline 3010 & 35 & 57 & 40.133 & -84 & 17 & 42.652 & GPS \\
\hline 3015 & 35 & 57 & 42.894 & -84 & 17 & 37.720 & GPS \\
\hline 3020 & 35 & 57 & 44.204 & -84 & 17 & 34.987 & GPS \\
\hline 3025 & 35 & 57 & 44.932 & -84 & 17 & 34.192 & GPS \\
\hline 3030 & 35 & 57 & 43.200 & -84 & 17 & 27.434 & GPS \\
\hline 3035 & 35 & 57 & 43.211 & -84 & 17 & 26.905 & GPS \\
\hline 3040 & 35 & 57 & 43.546 & -84 & 17 & 38.623 & GPS \\
\hline 3045 & 35 & 57 & 47.045 & -84 & 17 & 34.872 & GPS \\
\hline 3050 & 35 & 57 & 47.570 & -84 & 17 & 35.304 & GPS \\
\hline 3055 & 35 & 57 & 49.550 & -84 & 17 & 37.900 & GPS \\
\hline 3060 & 35 & 57 & 49.838 & -84 & 17 & 37.558 & GPS \\
\hline 3065 & 35 & 57 & 50.746 & -84 & 17 & 38.404 & GPS \\
\hline 3070 & 35 & 57 & 50.443 & -84 & 17 & 37.590 & GPS \\
\hline 3075 & 35 & 57 & 52.942 & -84 & 17 & 38.540 & GPS \\
\hline 3078 & 35 & 57 & 56.668 & -84 & 17 & 34.289 & GPS \\
\hline 3080 & 35 & 57 & 55.231 & -84 & 17 & 38.422 & GPS \\
\hline 3085 & 35 & 57 & 55.800 & -84 & 17 & 37.316 & GPS \\
\hline 3090 & 35 & 57 & 59.407 & -84 & 17 & 38.555 & GPS \\
\hline 3095 & 35 & 58 & 4.930 & -84 & 17 & 41.611 & GPS \\
\hline 3100 & 35 & 58 & 6.946 & -84 & 17 & 45.175 & GPS \\
\hline 3105 & 35 & 58 & 3.749 & -84 & 17 & 42.464 & GPS \\
\hline 3110 & 35 & 57 & 58.626 & -84 & 17 & 37.892 & GPS \\
\hline 3115 & 35 & 58 & 1.027 & -84 & 17 & 37.504 & GPS \\
\hline 3120 & 35 & 58 & 2.525 & -84 & 17 & 38.170 & GPS \\
\hline 3125 & 35 & 58 & 3.817 & -84 & 17 & 38.494 & GPS \\
\hline 3130 & 35 & 58 & 4.379 & -84 & 17 & 36.431 & GPS \\
\hline 3135 & 35 & 58 & 4.418 & -84 & 17 & 35.808 & GPS \\
\hline 3140 & 35 & 58 & 4.678 & -84 & 17 & 38.231 & GPS \\
\hline 3145 & 35 & 58 & 4.825 & -84 & 17 & 38.386 & DIG \\
\hline 3150 & 35 & 58 & 7.522 & -84 & 17 & 38.386 & GPS \\
\hline 3155 & 35 & 58 & 8.130 & -84 & 17 & 38.983 & GPS \\
\hline 3160 & 35 & 58 & 8.634 & -84 & 17 & 39.552 & GPS \\
\hline 3165 & 35 & 58 & 11.572 & -84 & 17 & 41.215 & GPS \\
\hline 3170 & 35 & 57 & 50.054 & -84 & 17 & 30.721 & GPS \\
\hline 3175 & 35 & 57 & 51.379 & -84 & 17 & 29.530 & GPS \\
\hline
\end{tabular}

10 Results of a Seepage Investigation at Bear Creek Valley, Oak Ridge, Tennessee, January through September 1994 
Table 2. State plane coordinates for seeps, springs, wetlands, and stream-measurement sites at Bear Creek Valley, Oak Ridge, Tennessee-Continued

\begin{tabular}{|c|c|c|c|c|c|c|c|}
\hline \multirow{3}{*}{$\begin{array}{c}\begin{array}{c}\text { Site } \\
\text { number }\end{array} \\
3180\end{array}$} & \multicolumn{6}{|c|}{ State plane coordinates } & \multirow{3}{*}{$\begin{array}{c}\text { Method } \\
\text { GPS }\end{array}$} \\
\hline & \multicolumn{3}{|c|}{$\begin{array}{c}\text { Latitude (N) } \\
\text { (degrees, minutes, } \\
\text { seconds) }\end{array}$} & \multicolumn{3}{|c|}{$\begin{array}{l}\text { Longitude (W) } \\
\text { (degrees, minutes, } \\
\text { seconds) }\end{array}$} & \\
\hline & 35 & 57 & 51.883 & -84 & 17 & 28.820 & \\
\hline 3185 & 35 & 57 & 53.446 & -84 & 17 & 28.669 & GPS \\
\hline 3190 & 35 & 57 & 55.541 & -84 & 17 & 25.350 & GPS \\
\hline 3195 & 35 & 57 & 59.479 & -84 & 17 & 26.646 & GPS \\
\hline 3198 & 35 & 58 & 4.696 & -84 & 17 & 25.998 & GPS \\
\hline 3200 & 35 & 58 & 3.904 & -84 & 17 & 27.254 & GPS \\
\hline 3202 & 35 & 58 & 5.621 & -84 & 17 & 25.544 & GPS \\
\hline 3205 & 35 & 58 & 7.201 & -84 & 17 & 27.409 & GPS \\
\hline 3210 & 35 & 58 & 8.148 & -84 & 17 & 27.776 & GPS \\
\hline 3215 & 35 & 58 & 10.589 & -84 & 17 & 25.076 & GPS \\
\hline 3225 & 35 & 58 & 13.105 & -84 & 17 & 27.488 & GPS \\
\hline 3230 & 35 & 58 & 15.946 & -84 & 17 & 24.659 & GPS \\
\hline 3235 & 35 & 58 & 19.430 & -84 & 17 & 24.320 & GPS \\
\hline 3240 & 35 & 58 & 8.681 & -84 & 17 & 28.356 & GPS \\
\hline 3245 & 35 & 58 & 10.877 & -84 & 17 & 29.656 & GPS \\
\hline 3250 & 35 & 58 & 10.848 & -84 & 17 & 30.325 & GPS \\
\hline 3255 & 35 & 58 & 11.453 & -84 & 17 & 31.628 & GPS \\
\hline 3260 & 35 & 58 & 16.514 & -84 & 17 & 34.584 & GPS \\
\hline 3265 & 35. & 58 & 17.605 & -84 & 17 & 35.272 & GPS \\
\hline 3270 & 35 & 58 & 13.123 & -84 & 17 & 29.119 & GPS \\
\hline 3275 & 35 & 58 & 16.734 & -84 & 17 & 29.792 & GPS \\
\hline 3280 & 35 & 58 & 18.203 & -84 & 17 & 30.286 & GPS \\
\hline 3285 & 35 & 57 & 55.102 & -84 & 17 & 23.053 & GPS \\
\hline 3290 & 35 & 57 & 51.678 & -84 & 17 & 19.136 & GPS \\
\hline 3295 & 35 & 57 & 49.234 & -84 & 17 & 14.899 & DIG \\
\hline 3300 & 35 & 57 & 48.816 & -84 & 17 & 15.320 & GPS \\
\hline 3305 & 35 & 57 & 47.909 & -84 & 17 & 6.925 & GPS \\
\hline 3310 & 35 & 57 & 57.614 & -84 & 17 & 18.337 & GPS \\
\hline 4005 & 35 & 57 & 21.524 & -84 & 18 & 13.586 & GPS \\
\hline 4010 & 35 & 57 & 28.919 & -84 & 18 & 16.106 & GPS \\
\hline 4015 & 35 & 57 & 29.776 & -84 & 18 & 12.038 & GPS \\
\hline 4017 & 35 & 57 & 31.450 & -84 & 18 & 14.278 & GPS \\
\hline 4020 & 35 & 57 & 29.480 & -84 & 18 & 16.880 & GPS \\
\hline 4025 & 35 & 57 & 31.288 & -84 & 18 & 21.254 & GPS \\
\hline 4030 & 35 & 57 & 30.298 & -84 & 18 & 16.193 & GPS \\
\hline 4035 & 35 & 57 & 32.209 & -84 & 18 & 15.617 & GPS \\
\hline 4040 & 35 & 57 & 35.716 & -84 & 18 & 10.022 & GPS \\
\hline 4045 & 35 & 57 & 38.390 & -84 & 18 & 11.999 & GPS \\
\hline 4050 & 35 & 57 & 37.739 & -84 & 18 & 13.882 & GPS \\
\hline 4055 & 35 & 57 & 40.576 & -84 & 18 & 17.176 & GPS \\
\hline
\end{tabular}

\begin{tabular}{|c|c|c|c|c|c|c|c|}
\hline \multirow{3}{*}{$\begin{array}{c}\begin{array}{c}\text { Site } \\
\text { number }\end{array} \\
4060\end{array}$} & \multicolumn{6}{|c|}{ State plane coordinates } & \multirow{3}{*}{$\begin{array}{c}\text { Method } \\
\text { GPS }\end{array}$} \\
\hline & \multicolumn{3}{|c|}{$\begin{array}{l}\text { Latitude }(\mathbf{N}) \\
\text { (degrees, minutes, } \\
\text { seconds) }\end{array}$} & \multicolumn{3}{|c|}{$\begin{array}{l}\text { Longitude (W) } \\
\text { (degrees, minutes, } \\
\text { seconds) }\end{array}$} & \\
\hline & 35 & 57 & 41.854 & -84 & 18 & 16.308 & \\
\hline 4065 & 35 & 57 & 44.273 & -84 & 18 & 18.832 & GPS \\
\hline 4070 & 35 & 57 & 43.430 & -84 & 18 & 14.386 & GPS \\
\hline 4075 & 35 & 57 & 48.992 & -84 & 18 & 17.798 & GPS \\
\hline 4080 & 35 & 57 & 37.908 & -84 & 18 & 23.062 & GPS \\
\hline 4085 & 35 & 57 & 38.009 & -84 & 18 & 23.123 & GPS \\
\hline 4090 & 35 & 57 & 43.747 & -84 & 18 & 26.986 & GPS \\
\hline 4095 & 35 & 57 & 42.494 & -84 & 18 & 21.737 & GPS \\
\hline 4100 & 35 & 57 & 45.248 & -84 & 18 & 23.267 & GPS \\
\hline 4105 & 35 & 57 & 20.218 & -84 & 18 & 10.958 & GPS \\
\hline 4110 & 35 & 57 & 23.029 & -84 & 18 & 7.945 & GPS \\
\hline 4115 & 35 & 57 & 26.143 & -84 & 18 & 3.510 & GPS \\
\hline 4120 & 35 & 57 & 32.699 & -84 & 17 & 32.964 & GPS \\
\hline 4125 & 35 & 57 & 33.343 & -84 & 17 & 38.598 & GPS \\
\hline 4400 & 35 & 57 & 27.997 & -84 & 18 & 3.017 & GPS \\
\hline 4405 & 35 & 57 & 28.228 & -84 & 18 & 2.524 & GPS \\
\hline 4410 & 35 & 57 & 28.598 & -84 & 18 & 2.246 & GPS \\
\hline 4415 & 35 & 57 & 30.442 & -84 & 18 & 1.228 & GPS \\
\hline 4420 & 35 & 57 & 30.510 & -84 & 18 & 0.986 & DIG \\
\hline 4425 & 35 & 57 & 29.747 & -84 & 18 & 2.570 & GPS \\
\hline 4430 & 35 & 57 & 32.357 & -84 & 18 & 3.089 & GPS \\
\hline 4435 & 35 & 57 & 35.161 & -84 & 18 & 2.473 & GPS \\
\hline 4440 & 35 & 57 & 39.751 & -84 & 18 & 1.192 & GPS \\
\hline 4445 & 35 & 57 & 42.178 & -84 & 17 & 59.464 & GPS \\
\hline 4450 & 35 & 57 & 41.515 & -84 & 18 & 1.544 & GPS \\
\hline 4455 & 35 & 57 & 43.391 & -84 & 18 & 2.426 & GPS \\
\hline 4472 & 35 & 57 & 58.878 & -84 & 18 & 1.987 & DIG \\
\hline 4473 & 35 & 57 & 59.267 & -84 & 18 & 1.904 & GPS \\
\hline 4475 & 35 & 57 & 35.244 & -84 & 18 & 2.621 & DIG \\
\hline 4480 & 35 & 57 & 40.349 & -84 & 18 & 7.931 & GPS \\
\hline 4485 & 35 & 57 & 44.734 & -84 & 18 & 8.748 & GPS \\
\hline 4490 & 35 & 57 & 48.125 & -84 & 18 & 8.572 & GPS \\
\hline 4495 & 35 & 57 & 48.269 & -84 & 18 & 9.209 & GPS \\
\hline 4500 & 35 & 57 & 49.050 & -84 & 18 & 9.533 & GPS \\
\hline 4505 & 35 & 57 & 49.000 & -84 & 18 & 9.533 & DIG \\
\hline 4510 & 35 & 57 & 51.203 & -84 & 18 & 10.062 & GPS \\
\hline 4515 & 35 & 57 & 52.556 & -84 & 18 & 10.890 & GPS \\
\hline 4520 & 35 & 57 & 52.556 & -84 & 18 & 10.890 & GPS \\
\hline 4525 & 35 & 57 & 33.772 & -84 & 17 & 54.787 & GPS \\
\hline 4530 & 35 & 57 & 36.778 & -84 & 17 & 50.687 & GPS \\
\hline
\end{tabular}


Table 2. State plane coordinates for seeps, springs, wetlands, and stream-measurement sites at Bear Creek Valley, Oak Ridge, Tennessee-Continued

\begin{tabular}{|c|c|c|c|c|c|c|c|}
\hline \multirow{3}{*}{$\begin{array}{c}\begin{array}{c}\text { Site } \\
\text { number }\end{array} \\
4535\end{array}$} & \multicolumn{6}{|c|}{ State plane coordinates } & \multirow{3}{*}{$\begin{array}{c}\text { Method } \\
\text { GPS }\end{array}$} \\
\hline & \multicolumn{3}{|c|}{$\begin{array}{l}\text { Latitude (N) } \\
\text { (degrees, minutes, } \\
\text { seconds) }\end{array}$} & \multicolumn{3}{|c|}{$\begin{array}{c}\text { Longitude (W) } \\
\text { (degrees, minutes, } \\
\text { seconds) }\end{array}$} & \\
\hline & 35 & 57 & 39.247 & -84 & 17 & 48.599 & \\
\hline 4540 & 35 & 57 & 43.459 & -84 & 17 & 50.136 & GPS \\
\hline 4545 & 35 & 57 & 48.301 & -84 & 17 & 51.882 & GPS \\
\hline 4580 & 35 & 57 & 56.740 & -84 & 17 & 52.091 & GPS \\
\hline 4585 & 35 & 57 & 56.758 & -84 & 17 & 52.699 & GPS \\
\hline 4590 & 35 & 57 & 56.311 & -84 & 17 & 50.798 & GPS \\
\hline 4595 & 35 & 57 & 59.638 & -84 & 17 & 50.852 & GPS \\
\hline 4600 & 35 & 58 & 2.795 & -84 & 17 & 47.818 & GPS \\
\hline 4605 & 35 & 58 & 0.977 & -84 & 17 & 51.094 & GPS \\
\hline 4610 & 35 & 58 & 2.323 & -84 & 17 & 51.119 & GPS \\
\hline 4615 & 35 & 58 & 3.018 & -84 & 17 & 51.817 & GPS \\
\hline 4620 & 35 & 58 & 5.603 & -84 & 17 & 51.702 & GPS \\
\hline 4630 & 35 & 57 & 39.614 & -84 & 17 & 46.982 & GPS \\
\hline 5005 & 35 & 57 & 9.227 & -84 & 18 & 33.152 & GPS \\
\hline 5010 & 35 & 57 & 10.994 & -84 & 18 & 29.516 & GPS \\
\hline 5015 & 35 & 57 & 18.767 & -84 & 18 & 33.066 & GPS \\
\hline 5020 & 35 & 57 & 25.546 & -84 & 18 & 39.791 & GPS \\
\hline 5025 & 35 & 57 & 34.535 & -84 & 18 & 41.022 & GPS \\
\hline 5030 & 35 & 57 & 13.889 & -84 & 18 & 25.909 & GPS \\
\hline 5035 & 35 & 57 & 14.188 & -84 & 18 & 25.726 & GPS \\
\hline 5040 & 35 & 57 & 16.463 & -84 & 18 & 25.837 & GPS \\
\hline 5045 & 35 & 57 & 22.424 & -84 & 18 & 30.809 & GPS \\
\hline 5050 & 35 & 57 & 31.396 & -84 & 18 & 32.429 & GPS \\
\hline 5055 & 35 & 57 & 31.334 & -84 & 18 & 32.396 & GPS \\
\hline 5060 & 35 & 57 & 33.779 & -84 & 18 & 35.406 & GPS \\
\hline 5065 & 35 & 57 & 30.712 & -84 & 18 & 35.302 & GPS \\
\hline 5070 & 35 & 57 & 33.880 & -84 & 18 & 31.784 & GPS \\
\hline 5075 & 35 & 57 & 34.499 & -84 & 18 & 31.946 & GPS \\
\hline 5080 & 35 & 57 & 40.212 & -84 & 18 & 32.609 & GPS \\
\hline 5085 & 35 & 57 & 35.338 & -84 & 18 & 30.737 & GPS \\
\hline 5090 & 35 & 57 & 17.636 & -84 & 18 & 21.578 & GPS \\
\hline 5095 & 35 & 57 & 26.554 & -84 & 18 & 22.406 & GPS \\
\hline 5100 & 35 & 57 & 30.359 & -84 & 18 & 25.880 & GPS \\
\hline 5105 & 35 & 57 & 18.508 & -84 & 18 & 17.514 & GPS \\
\hline 6005 & 35 & 57 & 0.612 & -84 & 18 & 59.234 & GPS \\
\hline 6007 & 35 & 57 & 1.458 & -84 & 18 & 59.922 & GPS \\
\hline 6010 & 35 & 57 & 5.494 & -84 & 18 & 58.658 & GPS \\
\hline 6015 & 35 & 57 & 6.138 & -84 & 19 & 1.168 & GPS \\
\hline 6020 & 35 & 57 & 9.349 & -84 & 19 & 4.739 & GPS \\
\hline 6025 & 35 & 57 & 8.953 & -84 & 18 & 59.792 & GPS \\
\hline
\end{tabular}

\begin{tabular}{|c|c|c|c|c|c|c|c|}
\hline \multirow{3}{*}{$\begin{array}{c}\begin{array}{c}\text { Site } \\
\text { number }\end{array} \\
6030\end{array}$} & \multicolumn{6}{|c|}{ State plane coordinates } & \multirow{3}{*}{$\begin{array}{c}\text { Method } \\
\text { GPS }\end{array}$} \\
\hline & \multicolumn{3}{|c|}{$\begin{array}{c}\text { Latitude }(\mathrm{N}) \\
\text { (degrees, minutes, } \\
\text { seconds) }\end{array}$} & \multicolumn{3}{|c|}{$\begin{array}{c}\text { Longitude (W) } \\
\text { (degrees, minutes, } \\
\text { seconds) }\end{array}$} & \\
\hline & 35 & 57 & 10.166 & -84 & 19 & 2.176 & \\
\hline 6033 & 35 & 57 & 15.487 & -84 & 18 & 59.508 & DIG \\
\hline 6035 & 35 & 57 & 15.340 & -84 & 18 & 59.674 & GPS \\
\hline 6045 & 35 & 57 & 23.350 & -84 & 19 & 8.695 & GPS \\
\hline 6050 & 35 & 57 & 20.164 & -84 & 19 & 0.613 & GPS \\
\hline 6055 & 35 & 57 & 25.513 & -84 & 18 & 57.535 & GPS \\
\hline 6060 & 35 & 57 & 0.752 & -84 & 18 & 58.295 & GPS \\
\hline 6065 & 35 & 57 & 3.809 & -84 & 18 & 51.710 & GPS \\
\hline 6070 & 35 & 57 & 4.284 & -84 & 18 & 50.576 & GPS \\
\hline 6075 & 35 & 57 & 7.283 & -84 & 18 & 47.880 & GPS \\
\hline 6085 & 35 & 57 & 12.838 & -84 & 18 & 50.728 & GPS \\
\hline 6090 & 35 & 57 & 9.749 & -84 & 18 & 45.997 & GPS \\
\hline 6095 & 35 & 57 & 11.275 & -84 & 18 & 43.038 & GPS \\
\hline 6100 & 35 & 57 & 13.406 & -84 & 18 & 47.117 & GPS \\
\hline 6105 & 35 & 57 & 13.633 & -84 & 18 & 47.232 & DIG \\
\hline 6110 & 35 & 57 & 19.040 & -84 & 18 & 47.765 & GPS \\
\hline 6115 & 35 & 57 & 22.536 & -84 & 18 & 48.949 & GPS \\
\hline 6120 & 35 & 57 & 29.804 & -84 & 18 & 50.987 & GPS \\
\hline 7005 & 35 & 57 & 0.515 & -84 & 18 & 47.117 & GPS \\
\hline 7010 & 35 & 56 & 56.774 & -84 & 18 & 47.416 & GPS \\
\hline 7015 & 35 & 56 & 51.896 & -84 & 18 & 44.687 & GPS \\
\hline 7020 & 35 & 56 & 48.106 & -84 & 18 & 45.022 & GPS \\
\hline 7025 & 35 & 56 & 47.692 & -84 & 18 & 41.548 & GPS \\
\hline 7030 & 35 & 56 & 43.670 & -84 & 18 & 40.716 & GPS \\
\hline 7035 & 35 & 56 & 41.820 & -84 & 18 & 40.280 & GPS \\
\hline 7040 & 35 & 56 & 40.762 & -84 & 18 & 33.980 & GPS \\
\hline 7045 & 35 & 56 & 35.092 & -84 & 18 & 36.576 & GPS \\
\hline 7050 & 35 & 57 & 5.713 & -84 & 18 & 39.614 & GPS \\
\hline 7055 & 35 & 57 & 9.148 & -84 & 18 & 40.381 & GPS \\
\hline 7060 & 35 & 57 & 5.922 & -84 & 18 & 38.333 & GPS \\
\hline 8005 & 35 & 56 & 40.214 & -84 & 19 & 26.954 & GPS \\
\hline 8010 & 35 & 56 & 45.830 & -84 & 19 & 30.137 & GPS \\
\hline 8015 & 35 & 56 & 52.789 & -84 & 19 & 29.863 & GPS \\
\hline 8020 & 35 & 56 & 52.390 & -84 & 19 & 33.672 & GPS \\
\hline 8025 & 35 & 56 & 52.922 & -84 & 19 & 34.374 & GPS \\
\hline 8030 & 35 & 56 & 53.221 & -84 & 19 & 40.109 & GPS \\
\hline 8035 & 35 & 56 & 57.404 & -84 & 19 & 42.550 & GPS \\
\hline 8040 & 35 & 56 & 51.126 & -84 & 19 & 43.741 & GPS \\
\hline 8045 & 35 & 56 & 59.302 & -84 & 19 & 33.985 & GPS \\
\hline 8050 & 35 & 56 & 59.827 & -84 & 19 & 33.557 & GPS \\
\hline
\end{tabular}

12 Results of a Seepage Investigation at Bear Creek Valley, Oak Ridge, Tennessee, January through September 1994 
Table 2. State plane coordinates for seeps, springs, wetlands, and stream-measurement sites at Bear Creek Valley, Oak Ridige, Tennessee-Continued

\begin{tabular}{|c|c|c|c|c|c|c|c|}
\hline \multirow{3}{*}{$\begin{array}{c}\begin{array}{c}\text { Site } \\
\text { number }\end{array} \\
8055\end{array}$} & \multicolumn{6}{|c|}{ State plane coordinates } & \multirow{3}{*}{$\begin{array}{c}\text { Method } \\
\text { GPS }\end{array}$} \\
\hline & \multicolumn{3}{|c|}{$\begin{array}{c}\text { Latitude }(\mathrm{N}) \\
\text { (degrees, minutes, } \\
\text { seconds) }\end{array}$} & \multicolumn{3}{|c|}{$\begin{array}{l}\text { Longitude (W) } \\
\text { (degrees, minutes, } \\
\text { seconds) }\end{array}$} & \\
\hline & 35 & 57 & 2.768 & -84 & 19 & 34.162 & \\
\hline 8060 & 35 & 57 & 3.647 & -84 & 19 & 34.219 & GPS \\
\hline 8062 & 35 & 57 & 8.060 & -84 & 19 & 36.160 & GPS \\
\hline 8063 & 35 & 57 & 8.532 & -84 & 19 & 35.969 & DIG \\
\hline 8065 & 35 & 57 & 12.118 & -84 & 19 & 37.582 & GPS \\
\hline 8070 & 35 & 57 & 12.683 & -84 & 19 & 42.776 & GPS \\
\hline 8075 & 35 & 57 & 11.135 & -84 & 19 & 45.577 & GPS \\
\hline 8080 & 35 & 57 & 12.218 & -84 & 19 & 46.153 & GPS \\
\hline 8085 & 35 & 57 & 16.441 & -84 & 19 & 50.542 & GPS \\
\hline 8090 & 35 & 57 & 12.406 & -84 & 19 & 37.132 & GPS \\
\hline 8092 & 35 & 57 & 13.194 & -84 & 19 & 36.671 & GPS \\
\hline 8095 & 35 & 57 & 15.030 & -84 & 19 & 36.840 & GPS \\
\hline 8100 & 35 & 57 & 15.768 & -84 & 19 & 36.116 & GPS \\
\hline 8103 & 35 & 57 & 15.581 & -84 & 19 & 36.671 & GPS \\
\hline 8105 & 35 & 57 & 16.556 & -84 & 19 & 36.959 & GPS \\
\hline 8110 & 35 & 57 & 19.598 & -84 & 19 & 43.259 & GPS \\
\hline 8115 & 35 & 57 & 20.405 & -84 & 19 & 34.734 & GPS \\
\hline 8120 & 35 & 57 & 21.740 & -84 & 19 & 39.464 & GPS \\
\hline 8125 & 35 & 57 & 20.318 & -84 & 19 & 34.144 & GPS \\
\hline 8130 & 35 & 57 & 22.234 & -84 & 19 & 32.200 & GPS \\
\hline & 25 & & & 84 & & & GPS \\
\hline 8135 & 35 & 57 & 22.831 & -84 & 19 & 27.678 & GPS \\
\hline 8140 & 35 & 57 & 20.070 & -84 & 19 & 24.712 & GPS \\
\hline 8145 & 35 & 57 & 20.959 & -84 & 19 & 18.386 & GPS \\
\hline 8150 & 35 & 57 & 21.701 & -84 & 19 & 20.870 & GPS \\
\hline 8200 & 35 & 56 & 39.959 & -84 & 19 & 25.975 & GPS \\
\hline 8205 & 35 & 57 & 13.475 & -84 & 19 & 31.098 & GPS \\
\hline 8210 & 35 & 57 & 18.263 & -84 & 19 & 33.218 & GPS \\
\hline 8220 & 35 & 57 & 17.140 & -84 & 19 & 28.978 & GPS \\
\hline 8225 & 35 & 57 & 14.580 & -84 & 19 & 29.064 & GPS \\
\hline 9005 & 35 & 56 & 46.334 & -84 & 19 & 19.092 & GPS \\
\hline 9010 & 35 & 56 & 49.085 & -84 & 19 & 16.270 & GPS \\
\hline 9015 & 35 & 56 & 51.644 & -84 & 19 & 15.942 & GPS \\
\hline 9020 & 35 & 56 & 52.084 & -84 & 19 & 16.277 & GPS \\
\hline 9025 & 35 & 56 & 54.164 & -84 & 19 & 20.118 & GPS \\
\hline 9030 & 35 & 56 & 59.006 & -84 & 19 & 22.670 & GPS \\
\hline 9035 & 35 & 57 & 0.184 & -84 & 19 & 23.326 & GPS \\
\hline 9040 & 35 & 56 & 52.847 & -84 & 19 & 16.230 & GPS \\
\hline 9045 & 35 & 56 & 59.708 & -84 & 19 & 11.773 & GPS \\
\hline 9050 & 35 & 57 & 1.508 & -84 & 19 & 17.180 & GPS \\
\hline 9055 & 35 & 57 & 4.889 & -84 & 19 & 17.314 & GPS \\
\hline
\end{tabular}

\begin{tabular}{|c|c|c|c|c|c|c|c|}
\hline \multirow{3}{*}{$\begin{array}{c}\begin{array}{c}\text { Site } \\
\text { number }\end{array} \\
9060\end{array}$} & \multicolumn{6}{|c|}{ State plane coordinates } & \multirow{3}{*}{$\begin{array}{c}\text { Method } \\
\text { GPS }\end{array}$} \\
\hline & \multicolumn{3}{|c|}{$\begin{array}{l}\text { Latitude (N) } \\
\text { (degrees, minutes, } \\
\text { seconds) }\end{array}$} & \multicolumn{3}{|c|}{$\begin{array}{l}\text { Longitude (W) } \\
\text { (degrees, minutes, } \\
\text { seconds) }\end{array}$} & \\
\hline & 35 & 57 & 3.823 & -84 & 19 & 24.460 & \\
\hline 9065 & 35 & 57 & 5.249 & -84 & 19 & 16.572 & GPS \\
\hline 9068 & 35 & 57 & 12.704 & -84 & 19 & 19.794 & GPS \\
\hline 9070 & 35 & 57 & 12.420 & -84 & 19 & 20.788 & GPS \\
\hline 9075 & 35 & 57 & 6.253 & -84 & 19 & 13.447 & GPS \\
\hline 9080 & 35 & 57 & 8.737 & -84 & 19 & 9.631 & GPS \\
\hline 9085 & 35 & 57 & 11.020 & -84 & 19 & 10.531 & GPS \\
\hline 9090 & 35 & 56 & 52.145 & -84 & 19 & 13.552 & GPS \\
\hline 9092 & 35 & 56 & 51.709 & -84 & 19 & 2.964 & GPS \\
\hline 9093 & 35 & 56 & 53.718 & -84 & 18 & 59.918 & GPS \\
\hline 9095 & 35 & 56 & 53.052 & .84 & 19 & 2.258 & GPS \\
\hline 10005 & 35 & 56 & 44.268 & -84 & 19 & 10.186 & GPS \\
\hline 10070 & 35 & 56 & 37.680 & -84 & 19 & 5.437 & GPS \\
\hline 10080 & 35 & 56 & 35.527 & -84 & 19 & 4.778 & GPS \\
\hline 10090 & 35 & 56 & 36.535 & -84 & 19 & 2.273 & GPS \\
\hline 10095 & 35 & 56 & 37.039 & -84 & 19 & 2.708 & GPS \\
\hline 10100 & 35 & 56 & 40.268 & -84 & 18 & 50.749 & GPS \\
\hline 11005 & 35 & 56 & 24.011 & -84 & 19 & 54.358 & GPS \\
\hline 11010 & 35 & 56 & 31.222 & -84 & 19 & 55.798 & GPS \\
\hline 11015 & 35 & 56 & 36.499 & -84 & 19 & 54.458 & GPS \\
\hline 11020 & 35 & 56 & 32.831 & -84 & 19 & 57.403 & GPS \\
\hline 11025 & 35 & 56 & 41.726 & -84 & 19 & 58.537 & GPS \\
\hline 11030 & 35 & 56 & 49.510 & -84 & 19 & 52.741 & GPS \\
\hline 11035 & 35 & 56 & 55.831 & -84 & 19 & 52.910 & GPS \\
\hline 11040 & 35 & 56 & 41.201 & -84 & 19 & 59.693 & GPS \\
\hline 11045 & 35 & 56 & 49.495 & -84 & 20 & 3.901 & GPS \\
\hline 11050 & 35 & 56 & 28.144 & -84 & 19 & 48.911 & GPS \\
\hline 11055 & 35 & 56 & 28.342 & -84 & 19 & 49.062 & GPS \\
\hline 11060 & 35 & 56 & 32.183 & -84 & 19 & 40.721 & GPS \\
\hline 11065 & 35 & 56 & 35.182 & -84 & 19 & 38.201 & GPS \\
\hline 11070 & 35 & 56 & 40.078 & -84 & 19 & 42.089 & GPS \\
\hline 11075 & 35 & 56 & 40.452 & -84 & 19 & 42.521 & GPS \\
\hline 11080 & 35 & 56 & 40.290 & -84 & 19 & 42.740 & GPS \\
\hline 11085 & 35 & 56 & 40.754 & -84 & 19 & 43.352 & GPS \\
\hline 11090 & 35 & 56 & 40.830 & -84 & 19 & 45.667 & GPS \\
\hline 11095 & 35 & 56 & 47.432 & -84 & 19 & 48.828 & GPS \\
\hline 11099 & 35 & 56 & 36.370 & -84 & 19 & 30.194 & GPS \\
\hline 11100 & 35 & 56 & 37.705 & -84 & 19 & 30.641 & GPS \\
\hline 11105 & 35 & 56 & 39.437 & -84 & 19 & 27.354 & GPS \\
\hline 12010 & 35 & 56 & 22.837 & -84 & 19 & 42.463 & GPS \\
\hline
\end{tabular}


Table 2. State plane coordinates for seeps, springs, wetlands, and stream-measurement sites at Bear Creek Valley, Oak Ridge, Tennessee-Continued

\begin{tabular}{|c|c|c|c|c|c|c|c|}
\hline \multirow{3}{*}{$\begin{array}{c}\begin{array}{c}\text { She } \\
\text { number }\end{array} \\
12015\end{array}$} & \multicolumn{6}{|c|}{ State plane coordinates } & \multirow{3}{*}{$\begin{array}{c}\text { Method } \\
\text { GPS }\end{array}$} \\
\hline & \multicolumn{3}{|c|}{$\begin{array}{l}\text { Latitude (N) } \\
\text { (degrees, minutes, } \\
\text { seconds) }\end{array}$} & \multicolumn{3}{|c|}{$\begin{array}{l}\text { Longltude (W) } \\
\text { (degrees, minutes, } \\
\text { seconds) }\end{array}$} & \\
\hline & 35 & 56 & 22.290 & -84 & 19 & 42.251 & \\
\hline 12020 & 35 & 56 & 20.494 & -84 & 19 & 40.148 & GPS \\
\hline 12025 & 35 & 56 & 19.417 & -84 & 19 & 37.862 & GPS \\
\hline 12040 & 35 & 56 & 16.980 & -84 & 19 & 26.796 & GPS \\
\hline 12045 & 35 & 56 & 20.036 & -84 & 19 & 16.352 & GPS \\
\hline 12050 & 35 & 56 & 5.798 & -84 & 19 & 43.507 & GPS \\
\hline 13005 & 35 & 56 & 33.479 & -84 & 20 & 38.530 & GPS \\
\hline 13007 & 35 & 56 & 30.919 & -84 & 20 & 36.344 & GPS \\
\hline 13008 & 35 & 56 & 34.674 & -84 & 20 & 34.397 & GPS \\
\hline 13010 & 35 & 56 & 35.639 & -84 & 20 & 32.374 & GPS \\
\hline 13015 & 35 & 56 & 34.138 & -84 & 20 & 30.905 & GPS \\
\hline 13020 & 35 & 56 & 35.790 & -84 & 20 & 27.208 & GPS \\
\hline 13025 & 35 & 56 & 30.343 & -84 & 20 & 35.873 & GPS \\
\hline 13030 & 35 & 56 & 29.069 & -84 & 20 & 34.264 & GPS \\
\hline 13035 & 35 & 56 & 28.734 & -84 & 20 & 29.936 & GPS \\
\hline 13040 & .35 & 56 & 28.918 & -84 & 20 & 36.301 & GPS \\
\hline 13060 & 35 & 56 & 26.776 & -84 & 20 & 34.476 & GPS \\
\hline 13070 & 35 & 56 & 20.465 & -84 & 20 & 38.947 & GPS \\
\hline 13075 & 35 & 56 & 19.255 & -84 & 20 & 43.699 & GPS \\
\hline 13080 & 35 & 56 & 24.785 & -84 & 20 & 52.544 & GPS \\
\hline 13085 & 35 & 56 & 16.753 & -84 & 20 & 49.549 & GPS \\
\hline 13086 & 35 & 56 & 17.552 & -84 & 20 & 50.485 & GPS \\
\hline 13087 & 35 & 56 & 16.624 & -84 & 20 & 49.456 & DIG \\
\hline 13088 & 35 & 56 & 14.402 & -84 & 20 & 50.240 & GPS \\
\hline 13089 & 35 & 56 & 12.088 & -84 & 20 & 49.164 & GPS \\
\hline 13090 & 35 & 56 & 14.201 & -84 & 20 & 55.154 & GPS \\
\hline 13095 & 35 & 56 & 15.770 & -84 & 20 & 56.634 & GPS \\
\hline 13140 & 35 & 56 & 12.566 & -84 & 20 & 57.109 & GPS \\
\hline 13150 & 35 & 56 & 22.412 & -84 & 21 & 3.690 & GPS \\
\hline 13155 & 35 & 56 & 12.638 & -84 & 20 & 58.960 & GPS \\
\hline 13160 & 35 & 56 & 12.271 & -84 & 20 & 59.129 & GPS \\
\hline 13165 & 35 & 56 & 10.525 & -84 & 20 & 59.305 & GPS \\
\hline 13170 & 35 & 56 & 11.393 & -84 & 21 & 0.842 & GPS \\
\hline 13175 & 35 & 56 & 18.146 & -84 & 21 & 5.911 & GPS \\
\hline 13180 & 35 & 56 & 6.918 & -84 & 21 & 7.877 & GPS \\
\hline 13200 & 35 & 56 & 10.334 & -84 & 21 & 16.520 & GPS \\
\hline 13205 & 35 & 56 & 25.854 & -84 & 20 & 33.248 & GPS \\
\hline 13210 & 35 & 56 & 26.189 & -84 & 20 & 26.171 & GPS \\
\hline 13215 & 35 & 56 & 26.498 & -84 & 20 & 22.355 & GPS \\
\hline 13220 & 35 & 56 & 27.672 & -84 & 20 & 23.780 & GPS \\
\hline
\end{tabular}

14 Results of a Seepage Investigation at Bear Creek Valley, Oak Ridge, Tennessee, January through September 1994

\begin{tabular}{|c|c|c|c|c|c|c|c|}
\hline \multirow{3}{*}{$\begin{array}{c}\begin{array}{c}\text { Site } \\
\text { number }\end{array} \\
13225\end{array}$} & \multicolumn{6}{|c|}{ State plane coordinates } & \multirow{3}{*}{$\begin{array}{c}\text { Method } \\
\text { GPS }\end{array}$} \\
\hline & \multicolumn{3}{|c|}{$\begin{array}{l}\text { Latitude (N) } \\
\text { (degrees, minutes, } \\
\text { seconds) }\end{array}$} & \multicolumn{3}{|c|}{$\begin{array}{l}\text { Longitude (W) } \\
\text { (degrees, minutes, } \\
\text { seconds) }\end{array}$} & \\
\hline & 35 & 56 & 29.807 & -84 & 20 & 23.244 & \\
\hline 13230 & 35 & 56 & 33.032 & -84 & 20 & 19.604 & GPS \\
\hline 13235 & 35 & 56 & 41.446 & -84 & 20 & 20.555 & GPS \\
\hline 13240 & 35 & 56 & 41.856 & -84 & 20 & 20.152 & GPS \\
\hline 13245 & 35 & 56 & 34.350 & -84 & 20 & 16.933 & GPS \\
\hline 13250 & 35 & 56 & 32.572 & -84 & 20 & 16.933 & DIG \\
\hline 13255 & 35 & 56 & 34.325 & -84 & 20 & 15.504 & GPS \\
\hline 13260 & 35 & 56 & 34.019 & -84 & 20 & 15.187 & DIG \\
\hline 13265 & 35 & 56 & 33.490 & -84 & 20 & 15.266 & GPS \\
\hline 13270 & 35 & 56 & 37.478 & -84 & 20 & 11.900 & GPS \\
\hline 13275 & 35 & 56 & 39.865 & -84 & 20 & 10.226 & GPS \\
\hline 13900 & 35 & 56 & 24.598 & -84 & 20 & 33.162 & GPS \\
\hline 14005 & 35 & 56 & 21.527 & -84 & 20 & 31.078 & GPS \\
\hline 14010 & 35 & 56 & 18.910 & -84 & 20 & 28.694 & GPS \\
\hline 14015 & 35 & 56 & 18.157 & -84 & 20 & 28.100 & GPS \\
\hline 14025 & 35 & 56 & 21.646 & -84 & 20 & 24.061 & GPS \\
\hline 14035 & 35 & 56 & 17.592 & -84 & 20 & 28.374 & GPS \\
\hline 14038 & 35 & 56 & 15.047 & -84 & 20 & 33.763 & GPS \\
\hline 14040 & 35 & 56 & 13.488 & -84 & 20 & 34.890 & GPS \\
\hline 14045 & 35 & 56 & 13.326 & -84 & 20 & 35.369 & GPS \\
\hline 14050 & 35 & 56 & 11.990 & -84 & 20 & 38.969 & GPS \\
\hline 14060 & 35 & 56 & 12.012 & -84 & 20 & 44.149 & GPS \\
\hline 14065 & 35 & 56 & 9.064 & -84 & 20 & 36.834 & GPS \\
\hline 14070 & 35 & 56 & 5.154 & -84 & 20 & 38.767 & GPS \\
\hline 14075 & 35 & 55 & 59.718 & -84 & 20 & 44.974 & GPS \\
\hline 14080 & 35 & 56 & 0.085 & -84 & 20 & 46.622 & GPS \\
\hline 14085 & 35 & 56 & 7.570 & -84 & 20 & 51.083 & GPS \\
\hline 14090 & 35 & 56 & 8.480 & -84 & 20 & 51.256 & GPS \\
\hline 14095 & 35 & 56 & 15.331 & -84 & 20 & 27.344 & GPS \\
\hline 14097 & 35 & 56 & 15.857 & -84 & 20 & 26.851 & GPS \\
\hline 14100 & 35 & 56 & 15.101 & -84 & 20 & 24.904 & GPS \\
\hline 14105 & 35 & 56 & 14.518 & -84 & 20 & 23.197 & GPS \\
\hline 14110 & 35 & 56 & 12.584 & -84 & 20 & 23.424 & GPS \\
\hline 14115 & 35 & 56 & 14.071 & -84 & 20 & 16.588 & GPS \\
\hline 14120 & 35 & 56 & 17.372 & -84 & 20 & 16.660 & GPS \\
\hline 14125 & 35 & 56 & 17.329 & -84 & 20 & 13.376 & GPS \\
\hline 14130 & 35 & 56 & 18.240 & -84 & 20 & 10.918 & GPS \\
\hline 14133 & 35 & 56 & 18.020 & -84 & 20 & 5.690 & GPS \\
\hline 14135 & 35 & 56 & 22.391 & -84 & 19 & 57.929 & GPS \\
\hline 14190 & 35 & 56 & 23.842 & -84 & 20 & 17.866 & GPS \\
\hline
\end{tabular}


Table 2. State plane coordinates for seeps, springs, wetlands, and stream-measurement sites at Bear Creek Valley, Oak Ridge, Tennessee-Continued

\begin{tabular}{|c|c|c|c|c|c|c|c|}
\hline \multirow{3}{*}{$\begin{array}{c}\begin{array}{c}\text { Site } \\
\text { number }\end{array} \\
14195\end{array}$} & \multicolumn{6}{|c|}{ State plane coordinates } & \multirow{3}{*}{$\begin{array}{c}\text { Method } \\
\text { GPS }\end{array}$} \\
\hline & \multicolumn{3}{|c|}{$\begin{array}{c}\text { Latitude }(\mathrm{N}) \\
\text { (degrees, minutes, } \\
\text { seconds) }\end{array}$} & \multicolumn{3}{|c|}{$\begin{array}{l}\text { Longitude (W) } \\
\text { (degrees, minutes, } \\
\text { seconds) }\end{array}$} & \\
\hline & 35 & 56 & 26.585 & -84 & 20 & 12.419 & \\
\hline 14200 & 35 & 56 & 28.680 & -84 & 20 & 8.520 & GPS \\
\hline 15005 & 35 & 56 & 2.126 & -84 & 20 & 28.936 & GPS \\
\hline 15010 & 35 & 55 & 57.580 & -84 & 20 & 27.560 & GPS \\
\hline 15015 & 35 & 55 & 55.178 & -84 & 20 & 25.840 & GPS \\
\hline 15020 & 35 & 55 & 54.354 & -84 & 20 & 25.710 & GPS \\
\hline 15025 & 35 & 55 & 52.932 & -84 & 20 & 25.127 & GPS \\
\hline 15030 & 35 & 55 & 49.051 & -84 & 20 & 25.415 & GPS \\
\hline 15035 & 35 & 55 & 54.566 & -84 & 20 & 25.163 & GPS \\
\hline 15040 & 35 & 55 & 53.710 & -84 & 20 & 24.529 & GPS \\
\hline 15045 & 35 & 55 & 52.756 & -84 & 20 & & GPS \\
\hline 15055 & 35 & 55 & 49.732 & -84 & 20 & 18 & GPS \\
\hline 15056 & 35 & 55 & 49.829 & -84 & 20 & & GPS \\
\hline 15065 & 35 & 55 & 44.6 & -84 & 20 & 18.215 & GPS \\
\hline 15070 & 35 & 55 & & -84 & 20 & 19.522 & GPS \\
\hline 15075 & 35 & 55 & & -84 & 20 & 16. & GPS \\
\hline 15080 & 35 & 55 & 52.068 & -84 & 20 & 10. & GPS \\
\hline 15081 & 35 & 55 & 52.0 & -84 & 20 & 10.788 & DIG \\
\hline 15085 & 35 & 55 & 56.942 & -84 & 20 & & GPS \\
\hline 16005 & 35 & 56 & 3.253 & -84 & 20 & 52.418 & GPS \\
\hline 16010 & 35 & 55 & 59.239 & -84 & 20 & 50.417 & GPS \\
\hline 16015 & 35 & 56 & 1.349 & -84 & 20 & 53.066 & GPS \\
\hline 16020 & 35 & 56 & 1.856 & -84 & 20 & 53.441 & GPS \\
\hline 16025 & 35 & 56 & 3.840 & -84 & 20 & 59.273 & GPS \\
\hline 16030 & 35 & 56 & 5.784 & -84 & 21 & 1.804 & GPS \\
\hline 16035 & 35 & 56 & 3.289 & -84 & 21 & 0.252 & GPS \\
\hline 16040 & 35 & 56 & 1.082 & -84 & 21 & 9.630 & GPS \\
\hline 16045 & 35 & 56 & 1.277 & -84 & 21 & 11.052 & GPS \\
\hline 16050 & 35 & 56 & 0.510 & -84 & 21 & 15.188 & GPS \\
\hline 16055 & 35 & 56 & 6.745 & -84 & 21 & 21.496 & GPS \\
\hline 16060 & 35 & 55 & 58.973 & -84 & 21 & 19.091 & GPS \\
\hline 16065 & 35 & 55 & 59.362 & -84 & 21 & 20.549 & GPS \\
\hline 16067 & 35 & 55 & 58.066 & -84 & 21 & 19.822 & GPS \\
\hline 16070 & 35 & 55 & 57.256 & -84 & 21 & 23.753 & GPS \\
\hline 16075 & 35 & 55 & 54.876 & -84 & 20 & 58.916 & GPS \\
\hline 16080 & 35 & 55 & 52.910 & -84 & 21 & 0.061 & GPS \\
\hline 16085 & 35 & 55 & 55.506 & -84 & 20 & 57.790 & GPS \\
\hline 16090 & 35 & 55 & 56.597 & -84 & 21 & 1.624 & GPS \\
\hline 16095 & 35 & 55 & 57.716 & -84 & 21 & 2.866 & GPS \\
\hline 16100 & 35 & 55 & 55.132 & -84 & 21 & 4.777 & GPS \\
\hline
\end{tabular}

\begin{tabular}{|c|c|c|c|c|c|c|c|}
\hline \multirow{3}{*}{$\begin{array}{c}\begin{array}{c}\text { Site } \\
\text { number }\end{array} \\
16105\end{array}$} & \multicolumn{6}{|c|}{ State plane coordinates } & \multirow{3}{*}{$\begin{array}{c}\text { Method } \\
\text { GPS }\end{array}$} \\
\hline & \multicolumn{3}{|c|}{$\begin{array}{l}\text { Latitude (N) } \\
\text { (degrees, minutes, } \\
\text { seconds) }\end{array}$} & \multicolumn{3}{|c|}{$\begin{array}{l}\text { Longitude (W) } \\
\text { (degrees, minutes, } \\
\text { seconds) }\end{array}$} & \\
\hline & 35 & 55 & 54.041 & -84 & 21 & 7.168 & \\
\hline 16110 & 35 & 55 & 59.074 & -84 & 21 & 14.677 & GPS \\
\hline 17005 & 35 & 55 & 26.742 & -84 & 21 & 35.269 & GPS \\
\hline 17010 & 35 & 55 & 28.337 & -84 & 21 & 34.841 & GPS \\
\hline 17015 & 35 & 55 & 35.353 & -84 & 21 & 28.192 & GPS \\
\hline 17020 & 35 & 55 & 38.978 & -84 & 21 & 21.445 & GPS \\
\hline 17030 & 35 & 55 & 43.046 & -84 & 21 & 21.514 & GPS \\
\hline 17035 & 35 & 55 & 47.348 & -84 & 21 & 27.882 & GPS \\
\hline 17040 & 35 & 55 & 44.944 & -84 & 21 & 21.506 & GPS \\
\hline 17045 & 35 & 55 & 46.888 & -84 & 21 & 23.245 & GPS \\
\hline 17050 & 35 & 55 & 44.818 & -84 & 21 & 20.657 & GPS \\
\hline 17055 & 35 & 55 & 52.450 & -84 & 21 & 21.949 & GPS \\
\hline 17065 & 35 & 55 & 45.570 & -84 & 21 & 13.057 & GPS \\
\hline 17070 & 35 & 55 & 34.277 & -84 & 21 & 35.644 & GPS \\
\hline 17075 & 35 & 55 & 35.738 & -84 & 21 & 34.456 & GPS \\
\hline 17082 & 35 & 55 & 38.896 & -84 & 21 & 31.140 & GPS \\
\hline 17083 & 35 & 55 & 39.227 & -84 & 21 & 32.062 & GPS \\
\hline 17085 & 35 & 55 & 37.067 & -84 & 21 & 35.118 & GPS \\
\hline 17090 & 35 & 55 & 40.062 & -84 & 21 & 40.144 & GPS \\
\hline 17095 & 35 & 55 & 36.836 & -84 & 21 & 34.567 & GPS \\
\hline 17100 & 35 & 55 & 38.784 & -84 & 21 & 34.430 & GPS \\
\hline 17105 & 35 & 55 & 46.085 & -84 & 21 & 34.870 & GPS \\
\hline 17110 & 35 & 55 & 46.906 & -84 & 21 & 35.572 & GPS \\
\hline 17115 & 35 & 55 & 47.719 & -84 & 21 & 35.795 & GPS \\
\hline 17120 & 35 & 55 & 48.907 & -84 & 21 & 37.296 & GPS \\
\hline 17125 & 35 & 55 & 49.775 & -84 & 21 & 34.758 & GPS \\
\hline 17130 & 35 & 55 & 56.525 & -84 & 21 & 39.841 & GPS \\
\hline 17135 & 35 & 55 & 51.139 & -84 & 21 & 34.650 & GPS \\
\hline 18005 & 35 & 55 & 13.498 & -84 & 21 & 56.261 & GPS \\
\hline 18010 & 35 & 55 & 21.011 & -84 & 22 & 0.599 & GPS \\
\hline 18015 & 35 & 55 & 22.969 & -84 & 21 & 57.780 & GPS \\
\hline 18020 & 35 & 55 & 21.792 & -84 & 22 & 1.445 & GPS \\
\hline 18025 & 35 & 55 & 26.267 & -84 & 22 & 2.863 & GPS \\
\hline 18060 & 35 & 55 & 13.649 & -84 & 21 & 55.260 & GPS \\
\hline 18065 & 35 & 55 & 7.910 & -84 & 21 & 48.956 & GPS \\
\hline 18070 & 35 & 55 & 7.655 & -84 & 21 & 48.272 & $\mathrm{DIG}$ \\
\hline 18075 & 35 & 55 & 7.518 & -84 & 21 & 48.449 & GPS \\
\hline 18080 & 35 & 55 & 7.655 & -84 & 21 & 48.272 & DIG \\
\hline 18085 & 35 & 55 & 7.208 & -84 & 21 & 46.976 & GPS \\
\hline 18090 & 35 & 55 & 7.493 & -84 & 21 & 44.726 & GPS \\
\hline
\end{tabular}

Table 215 
Table 2. State plane coordinates for seeps, springs, wetlands, and stream-measurement sites at Bear Creek Valley, Oak Ridge, Tennessee-Continued

\begin{tabular}{|c|c|c|c|c|c|c|c|}
\hline \multirow{3}{*}{$\begin{array}{c}\begin{array}{c}\text { Site } \\
\text { number }\end{array} \\
18095\end{array}$} & \multicolumn{6}{|c|}{ State plane coordinates } & \multirow{3}{*}{$\begin{array}{c}\text { Method } \\
\text { GPS }\end{array}$} \\
\hline & \multicolumn{3}{|c|}{$\begin{array}{l}\text { Latitude (N) } \\
\text { (degrees, minutes, } \\
\text { seconds) }\end{array}$} & \multicolumn{3}{|c|}{$\begin{array}{l}\text { Longitude (W) } \\
\text { (degrees, minutes, } \\
\text { seconds) }\end{array}$} & \\
\hline & 35 & 55 & 17.213 & -84 & 21 & 48.974 & \\
\hline 18100 & 35 & 55 & 17.569 & -84 & 21 & 49.662 & DIG \\
\hline 18105 & 35 & 55 & 17.692 & -84 & 21 & 49.554 & GPS \\
\hline 18110 & 35 & 55 & 26.900 & -84 & 21 & 44.766 & GPS \\
\hline 18115 & 35 & 55 & 29.086 & -84 & 21 & 44.543 & GPS \\
\hline 18120 & 35 & 55 & 30.086 & -84 & 21 & 42.476 & GPS \\
\hline 18125 & 35 & 55 & 31.498 & -84 & 21 & 40.889 & GPS \\
\hline 18130 & 35 & 55 & 34.522 & -84 & 21 & 43.002 & GPS \\
\hline 18140 & 35 & 55 & 38.993 & -84 & 21 & 44.006 & GPS \\
\hline 18145 & 35 & 55 & 21.396 & -84 & 21 & 50.382 & GPS \\
\hline 18150 & 35 & 55 & 26.299 & -84 & 21 & 53.460 & GPS \\
\hline 18155 & 35 & 55 & 31.422 & -84 & 21 & 53.075 & GPS \\
\hline 18160 & 35 & 55 & 34.957 & -84 & 21 & 53.618 & GPS \\
\hline 18165 & 35 & 55 & 35.731 & -84 & 21 & 54.029 & GPS \\
\hline 18170 & 35 & 55 & 36.296 & -84 & 21 & 54.479 & GPS \\
\hline 18175 & 35 & 55 & 36.851 & -84 & 21 & 57.640 & GPS \\
\hline 18180 & 35 & 55 & 36.804 & -84 & 21 & 55.530 & GPS \\
\hline 18185 & 35 & 55 & 40.609 & -84 & 21 & 50.353 & GPS \\
\hline 18190 & 35 & 55 & 41.088 & -84 & 21 & 51.484 & GPS \\
\hline 18195 & 35 & 55 & 41.682 & -84 & 21 & 51.646 & GPS \\
\hline 18215 & 35 & 55 & 44.490 & -84 & 21 & 52.189 & GPS \\
\hline 18220 & 35 & 55 & 48.104 & -84 & 21 & 55.876 & GPS \\
\hline 18225 & 35 & 55 & 41.873 & -84 & 21 & 49.482 & GPS \\
\hline 18230 & 35 & 55 & 44.796 & -84 & 21 & 45.605 & GPS \\
\hline 18250 & 35 & 55 & 18.030 & -84 & 21 & 48.690 & GPS \\
\hline 18255 & 35 & 55 & 19.639 & -84 & 21 & 44.611 & GPS \\
\hline 18260 & 35 & 55 & 23.423 & -84 & 21 & 39.107 & GPS \\
\hline 19003 & 35 & 54 & 54.781 & -84 & 22 & 19.549 & GPS \\
\hline 19004 & 35 & 54 & 56.322 & -84 & 22 & 18.386 & GPS \\
\hline 19005 & 35 & 54 & 57.118 & -84 & 22 & 21.464 & GPS \\
\hline 19007 & 35 & 54 & 57.776 & -84 & 22 & 15.730 & GPS \\
\hline 19008 & 35 & 54 & 59.134 & -84 & 22 & 14.905 & GPS \\
\hline 19010 & 35 & 55 & 0.203 & -84 & 22 & 12.713 & GPS \\
\hline 19015 & 35 & 55 & 1.225 & -84 & 22 & 12.040 & GPS \\
\hline 19045 & 35 & 55 & 6.650 & -84 & 22 & 12.536 & GPS \\
\hline 19050 & 35 & 55 & 9.898 & -84 & 22 & 17.170 & GPS \\
\hline 19055 & 35 & 55 & 16.619 & -84 & 22 & 21.630 & GPS \\
\hline 19090 & 35 & 55 & 7.813 & -84 & 22 & 10.196 & GPS \\
\hline 19095 & 35 & 55 & 9.887 & -84 & 22 & 13.156 & GPS \\
\hline 19100 & 35 & 55 & 11.395 & -84 & 22 & 13.166 & GPS \\
\hline
\end{tabular}

\begin{tabular}{ccc}
\hline \multirow{2}{*}{$\begin{array}{c}\text { Site } \\
\text { number }\end{array}$} & $\begin{array}{c}\text { Latitude (N) } \\
\text { (degrees, minutes, } \\
\text { seconds) }\end{array}$ & $\begin{array}{c}\text { Longitude (W) } \\
\text { (degrees, minutes, } \\
\text { seconds) }\end{array}$ \\
\hline
\end{tabular}

$\begin{array}{llllllll}19105 & 35 & 55 & 11.654 & -84 & 22 & 13.066 & \text { GPS } \\ 19110 & 35 & 55 & 16.626 & -84 & 22 & 7.331 & \text { GPS } \\ 19115 & 35 & 55 & 12.961 & -84 & 22 & 13.624 & \text { GPS } \\ 19120 & 35 & 55 & 16.594 & -84 & 22 & 17.396 & \text { GPS } \\ 19125 & 35 & 55 & 13.195 & -84 & 22 & 12.252 & \text { GPS } \\ 19130 & 35 & 55 & 17.540 & -84 & 22 & 6.305 & \text { GPS } \\ 19135 & 35 & 55 & 19.909 & -84 & 22 & 10.553 & \text { GPS } \\ 19140 & 35 & 55 & 20.788 & -84 & 22 & 11.543 & \text { GPS } \\ 19145 & 35 & 55 & 23.516 & -84 & 22 & 11.431 & \text { GPS } \\ 19146 & 35 & 55 & 25.201 & -84 & 22 & 11.053 & \text { GPS } \\ & & & & & & & \\ 19148 & 35 & 55 & 27.494 & -84 & 22 & 10.942 & \text { GPS } \\ 19150 & 35 & 55 & 22.062 & -84 & 22 & 8.231 & \text { GPS } \\ 19155 & 35 & 55 & 29.255 & -84 & 22 & 6.762 & \text { GPS } \\ 19156 & 35 & 55 & 29.453 & -84 & 22 & 6.758 & \text { DIG } \\ 19158 & 35 & 55 & 32.174 & -84 & 22 & 7.500 & \text { GPS } \\ 19160 & 35 & 55 & 32.956 & -84 & 22 & 3.446 & \text { GPS } \\ 19165 & 35 & 55 & 33.431 & -84 & 22 & 3.601 & \text { GPS } \\ 19170 & 35 & 55 & 33.992 & -84 & 22 & 2.827 & \text { GPS } \\ 19175 & 35 & 55 & 23.027 & -84 & 22 & 14.614 & \text { GPS } \\ 19180 & 35 & 55 & 27.840 & -84 & 22 & 15.305 & \text { GPS }\end{array}$

$\begin{array}{llllllll}19285 & 35 & 55 & 4.642 & -84 & 22 & 6.168 & \text { GPS } \\ 19290 & 35 & 55 & 11.132 & -84 & 22 & 0.167 & \text { GPS } \\ 19295 & 35 & 55 & 12.464 & -84 & 22 & 1.096 & \text { GPS } \\ 19300 & 35 & 55 & 12.731 & -84 & 21 & 57.496 & \text { GPS } \\ 20005 & 35 & 54 & 38.729 & -84 & 22 & 51.521 & \text { GPS } \\ 20010 & 35 & 54 & 43.960 & -84 & 22 & 45.656 & \text { GPS } \\ 20015 & 35 & 54 & 52.859 & -84 & 22 & 48.400 & \text { GPS } \\ 20020 & 35 & 54 & 58.226 & -84 & 22 & 54.808 & \text { GPS } \\ 20250 & 35 & 54 & 44.104 & -84 & 22 & 44.904 & \text { GPS } \\ 20255 & 35 & 54 & 46.076 & -84 & 22 & 37.772 & \text { GPS }\end{array}$

$\begin{array}{llcccccc}20258 & 35 & 54 & 48.218 & -84 & 22 & 33.409 & \text { GPS } \\ 20260 & 35 & 54 & 50.760 & -84 & 22 & 30.763 & \text { GPS } \\ 20265 & 35 & 54 & 50.998 & -84 & 22 & 30.029 & \text { GPS } \\ 20280 & 35 & 54 & 54.209 & -84 & 22 & 46.038 & \text { GPS } \\ 20285 & 35 & 55 & 0.012 & -84 & 22 & 38.597 & \text { GPS } \\ 20290 & 35 & 54 & 59.508 & -84 & 22 & 46.902 & \text { GPS } \\ 20295 & 35 & 55 & 0.595 & -84 & 22 & 46.564 & \text { GPS } \\ 20300 & 35 & 55 & 0.559 & -84 & 22 & 44.594 & \text { DIG } \\ 20305 & 35 & 55 & 3.508 & -84 & 22 & 45.293 & \text { GPS } \\ 20310 & 35 & 55 & 3.680 & -84 & 22 & 45.170 & \text { DIG }\end{array}$

16 Results of a Seepage Investigation at Bear Creek Valley, Oak Ridge, Tennessee, January through September 1994 
Table 2. State plane coordinates for seeps, springs, wetlands, and stream-measurement sites at Bear Creek Valley, Oak Ridge, Tennessee-Continued

\begin{tabular}{|c|c|c|c|c|c|c|c|c|c|c|c|c|c|c|c|}
\hline \multirow{3}{*}{$\begin{array}{c}\begin{array}{c}\text { Site } \\
\text { number }\end{array} \\
20315\end{array}$} & \multicolumn{6}{|c|}{ State plane coordinates } & \multirow{3}{*}{$\begin{array}{c}\text { Method } \\
\text { GPS }\end{array}$} & \multirow{3}{*}{$\begin{array}{c}\begin{array}{c}\text { Site } \\
\text { number }\end{array} \\
21035\end{array}$} & \multicolumn{6}{|c|}{ State plane coordinates } & \multirow{3}{*}{$\begin{array}{c}\text { Method } \\
\text { GPS }\end{array}$} \\
\hline & \multicolumn{3}{|c|}{$\begin{array}{l}\text { Latitude (N) } \\
\text { (degrees, minutes, } \\
\text { seconds) }\end{array}$} & \multicolumn{3}{|c|}{$\begin{array}{c}\text { Longitude (W) } \\
\text { (degrees, minutes, } \\
\text { seconds) }\end{array}$} & & & \multicolumn{3}{|c|}{$\begin{array}{c}\text { Latitude (N) } \\
\text { (degrees, minutes, } \\
\text { seconds) }\end{array}$} & \multicolumn{3}{|c|}{$\begin{array}{l}\text { Longitude (W) } \\
\text { (degrees, minutes, } \\
\text { seconds) }\end{array}$} & \\
\hline & 35 & 55 & 11.770 & -84 & 22 & 50.398 & & & 35 & 54 & 43.384 & -84 & 23 & 18.974 & \\
\hline 20320 & 35 & 55 & 4.523 & -84 & 22 & 41.588 & GPS & 21040 & 35 & 54 & 44.399 & -84 & 23 & 17.711 & GPS \\
\hline 20325 & 35 & 55 & 7.705 & -84 & 22 & 42.877 & GPS & 21045 & 35 & 54 & 45.295 & -84 & 23 & 17.092 & GPS \\
\hline 20335 & 35 & 55 & 13.562 & -84 & 22 & 43.108 & GPS & 21050 & 35 & 54 & 51.930 & .84 & 23 & 19.799 & GPS \\
\hline 20340 & 35 & 54 & 53.320 & -84 & 22 & 28.067 & GPS & 21055 & 35 & 54 & 50.011 & -84 & 23 & 12.728 & GPS \\
\hline 20342 & 35 & 54 & 56.016 & -84 & 22 & 24.931 & GPS & 21057 & 35 & 54 & 55.123 & -84 & 23 & 14.492 & GPS \\
\hline 20343 & 35 & 55 & 6.506 & -84 & 22 & 25.194 & GPS & 21058 & 35 & 54 & 56.434 & -84 & 23 & 12.772 & GPS \\
\hline 20350 & 35 & 54 & 58.637 & -84 & 22 & 31.217 & GPS & 21060 & 35 & 54 & 55.944 & -84 & 23 & 15.061 & GPS \\
\hline 20355 & 35 & 54 & 59.130 & -84 & 22 & 31.858 & GPS & 21065 & 35 & 54 & 51.070 & -84 & 23 & 11.087 & GPS \\
\hline 20360 & 35 & 55 & 9.520 & -84 & 22 & 32.704 & GPS & 21100 & 35 & 54 & 45.180 & -84 & 23 & 3.224 & GPS \\
\hline 20365 & 35 & 55 & 10.513 & -84 & 22 & 32.660 & GPS & 21102 & 35 & 54 & 47.516 & -84 & 23 & 2.576 & GPS \\
\hline 20370 & 35 & 55 & 10.967 & -84 & 22 & 33.737 & GPS & 21103 & 35 & 54 & 50.501 & -84 & 23 & 3.077 & GPS \\
\hline 20375 & 35 & 55 & 13.570 & -84 & 22 & 36.880 & GPS & 21105 & 35 & 54 & 53.010 & -84 & 23 & 2.681 & GPS \\
\hline 20380 & 35 & 55 & 11.842 & -84 & 22 & 35.620 & GPS & 21110 & 35 & 54 & 54.349 & -84 & 23 & 1.972 & GPS \\
\hline 20385 & 35 & 55 & 12.619 & -84 & 22 & 28.585 & GPS & 21115 & 35 & 54 & 55.976 & -84 & 23 & 1.378 & GPS \\
\hline 20390 & 35. & 55 & 13.998 & -84 & 22 & 30.227 & GPS & 21119 & 35 & 55 & 2.183 & -84 & 23 & 4.636 & DIG \\
\hline 20395 & 35 & 55 & 15.708 & -84 & 22 & 30.972 & GPS & 21120 & 35 & 55 & 2.186 & -84 & 23 & 4.639 & GPS \\
\hline 20400 & 35 & 55 & 19.772 & -84 & 22 & 34.799 & GPS & 21125 & 35 & 55 & 5.192 & -84 & 23 & 7.076 & GPS \\
\hline 20405 & 35 & 55 & 15.348 & -84 & 22 & 30.245 & GPS & 21130 & 35 & 55 & 8.270 & -84 & 23 & 7.015 & GPS \\
\hline 20410 & 35 & 55 & 18.397 & -84 & 22 & 28.726 & GPS & 22005 & 35 & 54 & 26.399 & -84 & 23 & 25.422 & GPS \\
\hline 20415 & 35 & 55 & 22.447 & -84 & 22 & 29.572 & GPS & 22006 & 35 & 54 & 28.292 & -84 & 23 & 24.108 & GPS. \\
\hline 20420 & 35 & 55 & 18.257 & -84 & 22 & 26.908 & GPS & 22010 & 35 & 54 & 23.616 & -84 & 23 & 22.992 & GPS \\
\hline 20425 & 35 & 55 & 20.784 & -84 & 22 & 23.408 & GPS & 22034 & 35 & 54 & 30.348 & -84 & 23 & 15.889 & GPS \\
\hline 20430 & 35 & 54 & 53.885 & -84 & 22 & 21.698 & GPS & 22040 & 35 & 54 & 32.108 & -84 & 23 & 8.941 & GPS \\
\hline 21005 & 35 & 54 & 34.225 & -84 & 23 & 24.828 & GPS & 22041 & 35 & 54 & 35.132 & -84 & 23 & 10.212 & GPS \\
\hline 21010 & 35 & 54 & 40.349 & -84 & 23 & 26.794 & GPS & 22042 & 35 & 54 & 40.201 & -84 & 23 & 10.378 & GPS \\
\hline 21015 & 35 & 54 & 34.690 & -84 & 23 & 23.266 & GPS & 22044 & 35 & 54 & 40.972 & -84 & 23 & 7.789 & GPS \\
\hline 21020 & 35 & 54 & 42.491 & -84 & 23 & 19.446 & GPS & 22045 & 35 & 54 & 35.471 & -84 & 22 & 58.231 & GPS \\
\hline 21025 & 35 & 54 & 42.721 & -84 & 23 & 18.895 & GPS & 22050 & 35 & 54 & 36.223 & -84 & 22 & 57.184 & GPS \\
\hline \multirow[t]{2}{*}{21030} & 35 & 54 & 49.907 & -84 & 23 & 11.530 & GPS & 22055 & 35 & 54 & 38.110 & -84 & 22 & 57.457 & GPS \\
\hline & & & & & & & & 22060 & 35 & 54 & 39.107 & -84 & 22 & 59.916 & GPS \\
\hline
\end{tabular}


Table 3. Discharge and water-quality data for the high base flow seepage investigation at Bear Creek Valley, Oak Ridge, Tennessee, March 14 through March 19, 1994

[Methods for measurement: D, no flowing water; E, estimation; F, float; L, less than mimimum reportable flow, 0.005 cubic foot per second; $P$, pygmy meter measurement; $R$, rating from stream gage; $V$, volumetric measurement; $\mathrm{ft}^{3} / \mathrm{s}$, cubic foot per second; $\mu \mathrm{S} / \mathrm{cm}$, microsiemens per centimeter; ${ }^{\circ} \mathrm{C}$, degree Celsius; $\mathrm{mg} / \mathrm{L}$, milligrams per liter; --, no datal

\begin{tabular}{|c|c|c|c|c|c|c|c|c|}
\hline $\begin{array}{c}\text { Site } \\
\text { number }\end{array}$ & $\begin{array}{l}\text { Type of } \\
\text { site }\end{array}$ & $\begin{array}{c}\text { Date } \\
\text { sampled } \\
\text { (month/ } \\
\text { day/year) }\end{array}$ & Method & $\begin{array}{l}\text { Flow } \\
\left(\mathrm{ft}^{3} / \mathrm{s}\right)\end{array}$ & pH & $\begin{array}{c}\text { Specific } \\
\text { conduc- } \\
\text { tance } \\
(\mu \mathrm{S} / \mathrm{cm})\end{array}$ & $\begin{array}{c}\text { Temper- } \\
\text { ature } \\
\left({ }^{\circ} \mathrm{C}\right)\end{array}$ & $\begin{array}{c}\text { Dis- } \\
\text { solved } \\
\text { oxygen } \\
\text { (mg/L) }\end{array}$ \\
\hline 1005 & Stream & $3 / 14 / 94$ & $\mathbf{P}$ & 0.57 & 7.4 & 902 & 8.0 & 11 \\
\hline 1010 & Stream & $3 / 14 / 94$ & $\mathrm{P}$ & .16 & 7.8 & 386 & 8.0 & $\begin{array}{l}11 \\
10.7\end{array}$ \\
\hline 1015 & Stream & $3 / 14 / 94$ & P & .15 & 7.8 & 379 & 9.0 & 10.1 \\
\hline 1020 & Stream & $3 / 14 / 94$ & $\mathrm{~F}$ & .01 & 7.9 & 265 & 12.0 & - \\
\hline 1025 & Stream & $3 / 14 / 94$ & V & .01 & 7.8 & 587 & 11.0 & 9.8 \\
\hline 1030 & Stream & $3 / 14 / 94$ & $\mathbf{F}$ & .01 & 6.9 & 125 & 8.5 & -- \\
\hline 1035 & Stream & $3 / 14 / 94$ & D & 0 & $\ldots$ & - & $\ldots$ & -- \\
\hline 1040 & Seep & $3 / 14 / 94$ & L & 0 & 6.9 & -- & 12.0 & 2.4 \\
\hline 1045 & Seep & $3 / 14 / 94$ & $\mathrm{v}$ & .01 & 7.5 & 88 & 10.0 & 9.4 \\
\hline 1050 & Stream & $3 / 14 / 94$ & $\mathbf{P}$ & .13 & 7.8 & 140 & 9.0 & -- \\
\hline 1055 & Seep & $3 / 14 / 94$ & D & 0 & -- & -- & .- & -- \\
\hline 1060 & Stream & $3 / 14 / 94$ & $F$ & .02 & 5.9 & 42 & 8.5 & -- \\
\hline 1065 & Seep & $3 / 14 / 94$ & L & 0 & 6 & 38 & 9.5 & 7.4 \\
\hline 1070 & Seep & $3 / 14 / 94$ & L & 0 & 6.7 & 40 & 8.5 & 10.8 \\
\hline 1075 & Stream & $3 / 14 / 94$ & $\bar{P}$ & .15 & 6.6 & 68 & 9.5 & - \\
\hline 1080 & Seep & $3 / 14 / 94$ & L & 0 & - & 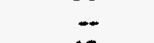 & - & -- \\
\hline 1085 & Stream & $3 / 14 / 94$ & F & .06 & 6.5 & 43 & 9.0 & -- \\
\hline 1090 & Stream & $3 / 14 / 94$ & L & 0 & 6.2 & 55 & 9.5 & -- \\
\hline 1095 & Seep & $3 / 14 / 94$ & $=$ & -- & 5.8 & 59 & 10.5 & 6.2 \\
\hline 1100 & Seep & $3 / 14 / 94$ & $\mathbf{P}$ & .03 & 5.6 & 37 & 9.5 & 6.4 \\
\hline 1105 & Stream & $3 / 14 / 94$ & $\mathbf{P}$ & .16 & 7.2 & 33 & 9.0 & -- \\
\hline 1110 & Stream & $3 / 14 / 94$ & E & .01 & 6.4 & 29 & 8.5 & - \\
\hline 1115 & Seep & $3 / 14 / 94$ & $\overline{\mathrm{L}}$ & 0 & 6.1 & 37 & 8.0 & 2.7 \\
\hline 1120 & Stream & $3 / 14 / 94$ & $\bar{L}$ & 0 & -- & -- & - & -- \\
\hline 1125 & Seep & $3 / 14 / 94$ & $\mathbf{L}$ & 0 & -- & -- & -- & -. \\
\hline 1130 & Stream & $3 / 14 / 94$ & E & .04 & 7.2 & 32 & 10.0 & - \\
\hline 1135 & Seep & $3 / 14 / 94$ & $\mathrm{E}$ & .01 & 6.7 & 38 & 11.0 & 9.7 \\
\hline 1140 & Stream & $3 / 14 / 94$ & $\mathbf{P}$ & 0.03 & 6.8 & 27 & 10.0 & -- \\
\hline 1145 & Stream & $3 / 14 / 94$ & $\mathbf{P}$ & .03 & 6.4 & 30 & 11.0 & -- \\
\hline 1148 & Stream & $3 / 14 / 94$ & E & .02 & 7 & 31 & 12.0 & -- \\
\hline 1150 & Spring & $3 / 14 / 94$ & E & .01 & 7.2 & 29 & 12.0 & -- \\
\hline 1155 & Seep & $3 / 14 / 94$ & E & .02 & 5.1 & 25 & 10.0 & 5 \\
\hline 1480 & Seep & $3 / 14 / 94$ & L & 0 & 7.4 & 457 & 10.0 & 8 \\
\hline 1485 & Stream & $3 / 14 / 94$ & $\overline{\mathbf{R}}$ & .44 & 7.5 & 239 & 10.0 & 11.2 \\
\hline 1490 & Seep & $3 / 14 / 94$ & $\ddot{E}$ & .01 & 7.2 & 646 & 7.0 & 3 \\
\hline 1495 & Stream & $3 / 14 / 94$ & P & .31 & 7.5 & 278 & 10.0 & 10.9 \\
\hline 1500 & Stream & $3 / 14 / 94$ & $\mathbf{P}$ & .3 & 8 & 1,350 & 10.0 & -- \\
\hline 1502 & Spring & $3 / 14 / 94$ & P & .07 & 7.6 & 954 & 13.0 & .6 \\
\hline 1505 & Stream & $3 / 14 / 94$ & $\mathrm{E}$ & .01 & 7.9 & 1,100 & 10.5 & -- \\
\hline 1510 & Stream & $3 / 14 / 94$ & $\mathbf{P}$ & .14 & 6.8 & 1,190 & 9.5 & -- \\
\hline 1515 & Stream & $3 / 14 / 94$ & $\mathbf{P}$ & .13 & 6.7 & 332 & 10.0 & -- \\
\hline 1520 & Seep & $3 / 14 / 94$ & L & 0 & 7.7 & 1,710 & 10.0 & $-\overline{2} 5$ \\
\hline 1525 & Spring & $3 / 14 / 94$ & E & .01 & 7.6 & 1,026 & 12.0 & \\
\hline 1530 & Seep & $3 / 14 / 94$ & L & 0 & 6.7 & 414 & 11.0 & $=$ \\
\hline 1535 & $\begin{array}{l}\text { Seep } \\
\text { Stream }\end{array}$ & $\begin{array}{l}3 / 14 / 94 \\
3 / 14 / 94\end{array}$ & $\begin{array}{l}\text { L } \\
P\end{array}$ & 0 & $\begin{array}{l}8.2 \\
8.1\end{array}$ & $\begin{array}{l}707 \\
162\end{array}$ & $\begin{array}{l}21.0 \\
14.0\end{array}$ & - \\
\hline $\begin{array}{l}1540 \\
1545\end{array}$ & $\begin{array}{l}\text { Stream } \\
\text { Seep }\end{array}$ & $\begin{array}{l}3 / 14 / 94 \\
3 / 14 / 94\end{array}$ & L & 0 & 6.6 & 180 & 9.0 & 4.7 \\
\hline 1550 & Stream & $3 / 14 / 94$ & $\mathbf{P}$ & .09 & 7 & 162 & 10.0 & -- \\
\hline 1555 & Stream & $3 / 14 / 94$ & $\mathbf{P}$ & .04 & 7.3 & 175 & 10.0 & $\because$ \\
\hline 1560 & Seep & $3 / 14 / 94$ & L & 0 & 6.8 & 50 & 11.0 & 8.4 \\
\hline
\end{tabular}


Table 3. Discharge and water-quality data for the high base flow seepage investigation at Bear Creek Valley, Oak Ridge, Tennessee, March 14 through March 19, 1994-Continued

\begin{tabular}{|c|c|c|c|c|c|c|c|c|}
\hline $\begin{array}{c}\text { Site } \\
\text { number }\end{array}$ & $\begin{array}{l}\text { Type of } \\
\text { site }\end{array}$ & $\begin{array}{c}\text { Date } \\
\text { sampled } \\
\text { (month/ } \\
\text { day/year) }\end{array}$ & Method & $\begin{array}{l}\text { Flow } \\
\left(\mathrm{ft}^{3} / \mathrm{s}\right)\end{array}$ & pH & $\begin{array}{c}\text { Specific } \\
\text { conduc- } \\
\text { tance } \\
(\mu \mathrm{S} / \mathrm{cm})\end{array}$ & $\begin{array}{c}\text { Temper- } \\
\text { ature } \\
\left({ }^{\circ} \mathrm{C}\right)\end{array}$ & $\begin{array}{c}\text { Dis- } \\
\text { solved } \\
\text { oxygen } \\
\text { (mg } / \text { ) }\end{array}$ \\
\hline $\begin{array}{l}1565 \\
1570 \\
1573 \\
1575 \\
1576 \\
1577 \\
1579 \\
1582 \\
1585 \\
1600\end{array}$ & $\begin{array}{l}\text { Spring } \\
\text { Stream } \\
\text { Stream } \\
\text { Seep } \\
\text { Seep } \\
\text { Seep } \\
\text { Stream } \\
\text { Stream } \\
\text { Spring } \\
\text { Stream }\end{array}$ & $\begin{array}{l}3 / 14 / 94 \\
3 / 14 / 94 \\
3 / 14 / 94 \\
3 / 14 / 94 \\
3 / 14 / 94 \\
3 / 14 / 94 \\
3 / 14 / 94 \\
3 / 14 / 94 \\
3 / 14 / 94 \\
3 / 14 / 94\end{array}$ & $\begin{array}{l}P \\
L \\
L \\
L \\
L \\
L \\
P \\
P \\
L \\
P\end{array}$ & $\begin{array}{l}.01 \\
0 \\
0 \\
0 \\
0 \\
0 \\
.02 \\
.03 \\
0 \\
.22\end{array}$ & $\begin{array}{l}6.7 \\
6.7 \\
6.5 \\
6.6 \\
7.2 \\
7.1 \\
6.9 \\
6.7 \\
6.9 \\
7.4\end{array}$ & $\begin{array}{r}50 \\
41 \\
33 \\
34 \\
135 \\
245 \\
179 \\
47 \\
60 \\
1,670\end{array}$ & $\begin{array}{r}10.0 \\
10.0 \\
14.0 \\
15.0 \\
14.0 \\
13.0 \\
11.0 \\
14.0 \\
13.0 \\
9.5\end{array}$ & $\begin{array}{l}6.7 \\
-- \\
-- \\
8.8 \\
6.2 \\
-- \\
-- \\
-- \\
8.9\end{array}$ \\
\hline $\begin{array}{l}1610 \\
2005 \\
2006 \\
2010 \\
2015 \\
2020 \\
2025 \\
2027 \\
2030 \\
2035\end{array}$ & $\begin{array}{l}\text { Seep } \\
\text { Stream } \\
\text { Seep } \\
\text { Seep } \\
\text { Stream } \\
\text { Stream } \\
\text { Stream } \\
\text { Seep } \\
\text { Stream } \\
\text { Stream }\end{array}$ & $\begin{array}{l}3 / 14 / 94 \\
3 / 15 / 94 \\
3 / 15 / 94 \\
3 / 15 / 94 \\
3 / 15 / 94 \\
3 / 15 / 94 \\
3 / 15 / 94 \\
3 / 15 / 94 \\
3 / 15 / 94 \\
3 / 15 / 94\end{array}$ & $\begin{array}{l}D \\
D \\
\text { L } \\
\text { L } \\
\text { P } \\
\text { P } \\
\text { D } \\
\text { D } \\
\text { P } \\
\text { L }\end{array}$ & $\begin{array}{l}0 \\
0 \\
0 \\
0 \\
.64 \\
.1 \\
0 \\
0 \\
.1 \\
0\end{array}$ & $\begin{array}{l}-- \\
-. \\
6.8 \\
6.6 \\
8.1 \\
7.7 \\
-- \\
- \\
7.4 \\
7.2\end{array}$ & $\begin{array}{r}-- \\
-- \\
126 \\
217 \\
524 \\
149 \\
-- \\
-- \\
126 \\
266\end{array}$ & $\begin{array}{c}-- \\
- \\
8.0 \\
8.0 \\
10.0 \\
9.0 \\
-- \\
-- \\
8.5 \\
9.0\end{array}$ & $\begin{array}{l}-- \\
-- \\
8.3 \\
5.5 \\
-- \\
-- \\
-- \\
-- \\
- \\
--\end{array}$ \\
\hline $\begin{array}{l}2040 \\
2050 \\
2055 \\
2065 \\
2067 \\
2068 \\
2070 \\
2080 \\
2083 \\
2085\end{array}$ & $\begin{array}{l}\text { Seep } \\
\text { Stream } \\
\text { Seep } \\
\text { Stream } \\
\text { Seep } \\
\text { Seep } \\
\text { Stream } \\
\text { Seep } \\
\text { Stream } \\
\text { Seep }\end{array}$ & $\begin{array}{l}3 / 15 / 94 \\
3 / 15 / 94 \\
3 / 15 / 94 \\
3 / 15 / 94 \\
3 / 15 / 94 \\
3 / 15 / 94 \\
3 / 15 / 94 \\
3 / 15 / 94 \\
3 / 15 / 94 \\
3 / 15 / 94\end{array}$ & $\begin{array}{l}\text { L } \\
\text { D } \\
\text { D } \\
\text { E } \\
\text { L } \\
\text { P } \\
D \\
D \\
D \\
D\end{array}$ & 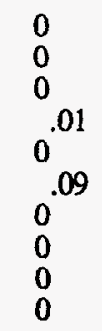 & $\begin{array}{l}-- \\
-- \\
-7.6 \\
7.5 \\
7.4 \\
-- \\
-- \\
-- \\
--\end{array}$ & $\begin{array}{r}545 \\
- \\
- \\
167 \\
67 \\
130 \\
- \\
- \\
- \\
-\end{array}$ & $\begin{array}{l}13.0 \\
-- \\
- \\
11.0 \\
13.5 \\
9.0 \\
- \\
- \\
-\end{array}$ & $\begin{array}{l}-- \\
-- \\
-- \\
- \\
-- \\
-- \\
-- \\
-\end{array}$ \\
\hline $\begin{array}{l}2090 \\
2095 \\
2100 \\
2110 \\
2120 \\
2125 \\
2130 \\
2135 \\
2140 \\
2145\end{array}$ & $\begin{array}{l}\text { Stream } \\
\text { Seep } \\
\text { Stream } \\
\text { Seep } \\
\text { Seep } \\
\text { Stream } \\
\text { Seep } \\
\text { Stream } \\
\text { Stream } \\
\text { Stream }\end{array}$ & $\begin{array}{l}3 / 15 / 94 \\
3 / 15 / 94 \\
3 / 15 / 94 \\
3 / 15 / 94 \\
3 / 15 / 94 \\
3 / 15 / 94 \\
3 / 15 / 94 \\
3 / 15 / 94 \\
3 / 15 / 94 \\
3 / 15 / 94\end{array}$ & $\begin{array}{l}\mathbf{D} \\
\mathbf{D} \\
\mathbf{F} \\
\mathbf{D} \\
\mathbf{D} \\
\mathbf{E} \\
\mathbf{E} \\
\mathbf{P} \\
\mathbf{E} \\
\mathbf{D}\end{array}$ & $\begin{array}{l}0 \\
0 \\
.02 \\
0 \\
0 \\
.04 \\
.01 \\
.07 \\
.01 \\
0^{.01}\end{array}$ & $\begin{array}{l}-- \\
\overline{7.2} \\
-- \\
- \\
7.2 \\
6.8 \\
6.8 \\
5.6 \\
-.\end{array}$ & $\begin{array}{l}-- \\
-- \\
77 \\
-- \\
-- \\
74 \\
50 \\
50 \\
32 \\
--\end{array}$ & $\begin{array}{l}-- \\
-\overline{9} .0 \\
-- \\
- \\
10.5 \\
11.5 \\
12.5 \\
10.0 \\
--\end{array}$ & $\begin{array}{l}-- \\
-- \\
-- \\
-- \\
-- \\
-- \\
9.2 \\
- \\
-- \\
--\end{array}$ \\
\hline $\begin{array}{l}2150 \\
2155 \\
2160 \\
2170 \\
2175 \\
2180 \\
2185 \\
2190 \\
2195 \\
2200\end{array}$ & $\begin{array}{l}\text { Spring } \\
\text { Stream } \\
\text { Spring } \\
\text { Stream } \\
\text { Stream } \\
\text { Seep } \\
\text { Stream } \\
\text { Stream } \\
\text { Stream } \\
\text { Stream }\end{array}$ & $\begin{array}{l}3 / 15 / 94 \\
3 / 15 / 94 \\
3 / 15 / 94 \\
3 / 15 / 94 \\
3 / 15 / 94 \\
3 / 15 / 94 \\
3 / 15 / 94 \\
3 / 15 / 94 \\
3 / 15 / 94 \\
3 / 15 / 94\end{array}$ & $\begin{array}{l}\text { L } \\
\text { P } \\
\text { E } \\
\text { L } \\
\text { D } \\
\text { D } \\
\text { P } \\
\text { P } \\
\text { P } \\
\text { D }\end{array}$ & $\begin{array}{l}0 \\
.04 \\
.01 \\
0 \\
0 \\
0 \\
.7 \\
.9 \\
1.16 \\
0\end{array}$ & $\begin{array}{l}-\overline{7} \\
6.6 \\
8.2 \\
- \\
- \\
8.3 \\
8.2 \\
8.1 \\
8.2\end{array}$ & $\begin{array}{r}-- \\
50 \\
64 \\
162 \\
- \\
-\overline{590} \\
605 \\
400 \\
760\end{array}$ & $\begin{array}{c}\overline{14.0} \\
11.5 \\
12.5 \\
- \\
\overline{12.0} \\
12.5 \\
12.5 \\
9.0\end{array}$ & $\begin{array}{l}-- \\
-\overline{9.7} \\
-- \\
- \\
- \\
-- \\
-- \\
- \\
-\end{array}$ \\
\hline
\end{tabular}


Table 3. Discharge and water-quality data for the high base flow seepage investigation at Bear Creek Valley,

Oak Ridge, Tennessee, March 14 through March 19, 1994-Continued

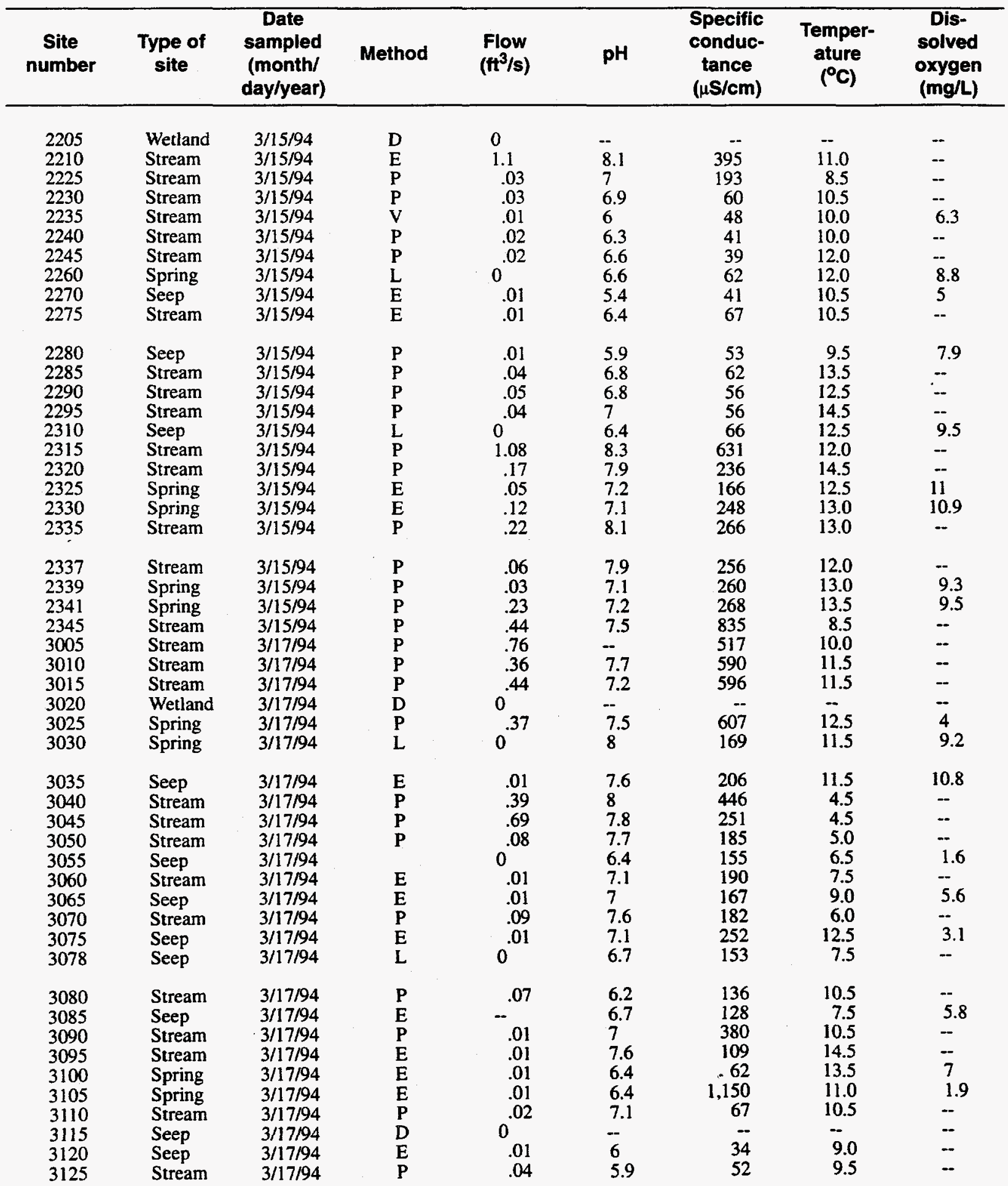


Table 3. Discharge and water-quality data for the high base flow seepage investigation at Bear Creek Valley, Oak Ridge, Tennessee, March 14 through March 19, 1994-Continued

\begin{tabular}{|c|c|c|c|c|c|c|c|c|}
\hline $\begin{array}{c}\text { Site } \\
\text { number }\end{array}$ & $\begin{array}{l}\text { Type of } \\
\text { site }\end{array}$ & $\begin{array}{c}\text { Date } \\
\text { sampled } \\
\text { (month/ } \\
\text { day/year) }\end{array}$ & Method & $\begin{array}{l}\text { Flow } \\
\left(\mathrm{ft}^{3} / \mathrm{s}\right)\end{array}$ & pH & $\begin{array}{c}\text { Specific } \\
\text { conduc- } \\
\text { tance } \\
(\mu \mathrm{S} / \mathrm{cm})\end{array}$ & $\begin{array}{c}\text { Temper- } \\
\text { ature } \\
\left({ }^{\circ} \mathrm{C}\right)\end{array}$ & $\begin{array}{c}\text { Dis- } \\
\text { solved } \\
\text { oxygen } \\
\text { (mg/L) }\end{array}$ \\
\hline $\begin{array}{l}3130 \\
3135 \\
3140 \\
3145 \\
3150 \\
3155 \\
3160 \\
3165 \\
3170 \\
3175\end{array}$ & $\begin{array}{l}\text { Seep } \\
\text { Seep } \\
\text { Seep } \\
\text { Seep } \\
\text { Seep } \\
\text { Seep } \\
\text { Stream } \\
\text { Spring } \\
\text { Stream } \\
\text { Seep }\end{array}$ & $\begin{array}{l}3 / 17 / 94 \\
3 / 17 / 94 \\
3 / 17 / 94 \\
3 / 17 / 94 \\
3 / 17 / 94 \\
3 / 17 / 94 \\
3 / 17 / 94 \\
3 / 17 / 94 \\
3 / 17 / 94 \\
3 / 17 / 94\end{array}$ & $\begin{array}{l}D \\
D \\
L \\
E \\
E \\
D \\
P \\
E \\
P \\
P\end{array}$ & $\begin{array}{l}0 \\
0 \\
0 \\
.01 \\
.01 \\
0 \\
.02 \\
.02 \\
.64 \\
.13\end{array}$ & \begin{tabular}{l}
-- \\
\hdashline 5.4 \\
5.4 \\
5.7 \\
-6.6 \\
6.2 \\
7.8 \\
7.2
\end{tabular} & $\begin{array}{r}-- \\
-- \\
63 \\
47 \\
33 \\
-- \\
45 \\
49 \\
499 \\
515\end{array}$ & $\begin{array}{c}-- \\
- \\
8.5 \\
10.5 \\
10.5 \\
- \\
11.0 \\
11.0 \\
6.0 \\
11.0\end{array}$ & $\begin{array}{l}-- \\
\ddot{4} \\
4.3 \\
6.2 \\
-- \\
-\overline{9.7} \\
\overline{7.8}\end{array}$ \\
\hline $\begin{array}{l}3180 \\
3185 \\
3190 \\
3195 \\
3198 \\
3200 \\
3202 \\
3205 \\
3210 \\
3215\end{array}$ & $\begin{array}{l}\text { Spring } \\
\text { Stream } \\
\text { Stream } \\
\text { Stream } \\
\text { Wetland } \\
\text { Stream } \\
\text { Stream } \\
\text { Seep } \\
\text { Stream } \\
\text { Seep }\end{array}$ & $\begin{array}{l}3 / 17 / 94 \\
3 / 17 / 94 \\
3 / 17 / 94 \\
3 / 17 / 94 \\
3 / 17 / 94 \\
3 / 17 / 94 \\
3 / 17 / 94 \\
3 / 17 / 94 \\
3 / 17 / 94 \\
3 / 17 / 94\end{array}$ & $\begin{array}{l}P \\
P \\
P \\
P \\
L \\
P \\
-- \\
L \\
P \\
D\end{array}$ & $\begin{array}{l}.13 \\
.5 \\
.14 \\
.1 \\
0 \\
.12 \\
.0 \\
0.03 \\
0\end{array}$ & $\begin{array}{l}7.1 \\
8 \\
7.4 \\
7.3 \\
6.7 \\
7.2 \\
7 \\
7.3 \\
7 \\
--\end{array}$ & $\begin{array}{r}513 \\
495 \\
116 \\
101 \\
160 \\
87 \\
348 \\
243 \\
45 \\
--\end{array}$ & $\begin{array}{r}11.0 \\
6.5 \\
7.0 \\
10.5 \\
19.5 \\
12.5 \\
12.0 \\
12.5 \\
10.5 \\
\ldots\end{array}$ & $\begin{array}{l}6.3 \\
-- \\
-- \\
-- \\
-- \\
-- \\
-- \\
-- \\
- \\
-\end{array}$ \\
\hline $\begin{array}{l}3225 \\
3230 \\
3235 \\
3240 \\
3245 \\
3250 \\
3255 \\
3260 \\
3265 \\
3270\end{array}$ & $\begin{array}{l}\text { Stream } \\
\text { Seep } \\
\text { Seep } \\
\text { Stream } \\
\text { Seep } \\
\text { Stream } \\
\text { Seep } \\
\text { Stream } \\
\text { Seep } \\
\text { Stream }\end{array}$ & $\begin{array}{l}3 / 17 / 94 \\
3 / 17 / 94 \\
3 / 17 / 94 \\
3 / 17 / 94 \\
3 / 17 / 94 \\
3 / 17 / 94 \\
3 / 17 / 94 \\
3 / 17 / 94 \\
3 / 17 / 94 \\
3 / 17 / 94\end{array}$ & $\begin{array}{l}\mathbf{L} \\
\mathrm{D} \\
\mathrm{D} \\
\mathbf{P} \\
\mathrm{E} \\
\mathrm{E} \\
\mathbf{D} \\
\mathrm{E} \\
\mathbf{L} \\
\mathbf{E}\end{array}$ & $\begin{array}{l}0 \\
0 \\
0 \\
.03 \\
.01 \\
.01 \\
0 \\
.01 \\
0 \\
.03\end{array}$ & $\begin{array}{l}5.6 \\
-- \\
-- \\
7.2 \\
5.7 \\
5.7 \\
-- \\
5.7 \\
-\overline{7.2}\end{array}$ & $\begin{array}{c}33 \\
- \\
- \\
51 \\
31 \\
35 \\
-- \\
24 \\
-- \\
48\end{array}$ & $\begin{array}{c}9.0 \\
-- \\
- \\
9.5 \\
9.0 \\
10.0 \\
-- \\
10.5 \\
\overline{9} .5\end{array}$ & $\begin{array}{l}-- \\
-- \\
-- \\
-- \\
5.5 \\
-- \\
-- \\
-- \\
-- \\
--\end{array}$ \\
\hline $\begin{array}{l}3275 \\
3280 \\
3285 \\
3290 \\
3295 \\
3300 \\
3305 \\
3310 \\
4005 \\
4010\end{array}$ & $\begin{array}{l}\text { Stream } \\
\text { Spring } \\
\text { Stream } \\
\text { Stream } \\
\text { Seep } \\
\text { Spring } \\
\text { Seep } \\
\text { Stream } \\
\text { Stream } \\
\text { Stream }\end{array}$ & $\begin{array}{l}3 / 17 / 94 \\
3 / 17 / 94 \\
3 / 17 / 94 \\
3 / 17 / 94 \\
3 / 17 / 94 \\
3 / 17 / 94 \\
3 / 17 / 94 \\
3 / 17 / 94 \\
3 / 18 / 94 \\
3 / 18 / 94\end{array}$ & $\begin{array}{l}\mathbf{P} \\
\mathbf{E} \\
\mathbf{P} \\
\mathbf{E} \\
\mathbf{L} \\
\mathbf{L} \\
\mathbf{L} \\
\mathbf{P} \\
\mathbf{P} \\
\mathbf{L}\end{array}$ & $\begin{array}{l}.03 \\
.03 \\
.4 \\
.01 \\
0 \\
0 \\
0 \\
.36 \\
.1 \\
0\end{array}$ & $\begin{array}{l}7.3 \\
7 \\
8.3 \\
8.2 \\
7.4 \\
7 \\
5.4 \\
8.3 \\
8.3 \\
6.4\end{array}$ & $\begin{array}{r}54 \\
66 \\
574 \\
150 \\
164 \\
146 \\
17 \\
584 \\
142 \\
52\end{array}$ & $\begin{array}{r}11.0 \\
11.5 \\
8.5 \\
12.5 \\
11.5 \\
12.0 \\
11.5 \\
12.5 \\
7.0 \\
8.5\end{array}$ & $\begin{array}{c}-\overline{10} \\
-- \\
- \\
8.2 \\
8.5 \\
9.4 \\
- \\
-- \\
--\end{array}$ \\
\hline $\begin{array}{l}4015 \\
4020 \\
4025 \\
4030 \\
4035 \\
4040 \\
4045 \\
4050 \\
4055 \\
4060\end{array}$ & $\begin{array}{l}\text { Seep } \\
\text { Stream } \\
\text { Seep } \\
\text { Stream } \\
\text { Stream } \\
\text { Seep } \\
\text { Seep } \\
\text { Seep } \\
\text { Stream } \\
\text { Seep }\end{array}$ & $\begin{array}{l}3 / 18 / 94 \\
3 / 18 / 94 \\
3 / 18 / 94 \\
3 / 18 / 94 \\
3 / 18 / 94 \\
3 / 18 / 94 \\
3 / 18 / 94 \\
3 / 18 / 94 \\
3 / 18 / 94 \\
3 / 18 / 94\end{array}$ & $\begin{array}{l}D \\
D \\
D \\
P \\
P \\
D \\
D \\
D \\
E \\
D\end{array}$ & $\begin{array}{l}0 \\
0 \\
0 \\
.09 \\
.06 \\
0 \\
0 \\
0 \\
.01 \\
0\end{array}$ & $\begin{array}{l}-. \\
-- \\
-- \\
8 \\
6.9 \\
-. \\
-- \\
\overline{5} .8 \\
--\end{array}$ & $\begin{array}{c}-- \\
- \\
- \\
85 \\
64 \\
-- \\
-- \\
-- \\
27 \\
--\end{array}$ & $\begin{array}{c}-- \\
- \\
- \\
8.5 \\
10.5 \\
- \\
-- \\
-- \\
11.5 \\
--\end{array}$ & $\begin{array}{l}-- \\
- \\
- \\
-- \\
- \\
- \\
- \\
- \\
- \\
-\end{array}$ \\
\hline
\end{tabular}


Table 3. Discharge and water-quality data for the high base flow seepage investigation at Bear Creek Valley,

Oak Ridge, Tennessee, March 14 through March 19, 1994-Continued

\begin{tabular}{|c|c|c|c|c|c|c|c|c|}
\hline $\begin{array}{c}\text { Site } \\
\text { number }\end{array}$ & $\begin{array}{l}\text { Type of } \\
\text { site }\end{array}$ & $\begin{array}{c}\text { Date } \\
\text { sampled } \\
\text { (month/ } \\
\text { day/year) }\end{array}$ & Method & $\begin{array}{l}\text { Flow } \\
\left(\mathrm{ft}^{3 / \mathrm{s}}\right)\end{array}$ & pH & $\begin{array}{c}\text { Specific } \\
\text { conduc- } \\
\text { tance } \\
(\mu S / \mathrm{cm})\end{array}$ & $\begin{array}{c}\text { Temper- } \\
\text { ature } \\
\left({ }^{\circ} \mathrm{C}\right)\end{array}$ & $\begin{array}{c}\text { Dis- } \\
\text { solved } \\
\text { oxygen } \\
\text { (mg/L) }\end{array}$ \\
\hline $\begin{array}{l}4065 \\
4070 \\
4075 \\
4080 \\
4085 \\
4090 \\
4095 \\
4100 \\
4105 \\
4110\end{array}$ & $\begin{array}{l}\text { Seep } \\
\text { Seep } \\
\text { Spring } \\
\text { Stream } \\
\text { Stream } \\
\text { Seep } \\
\text { Seep } \\
\text { Stream } \\
\text { Spring } \\
\text { Stream }\end{array}$ & $\begin{array}{l}3 / 18 / 94 \\
3 / 18 / 94 \\
3 / 18 / 94 \\
3 / 18 / 94 \\
3 / 18 / 94 \\
3 / 18 / 94 \\
3 / 18 / 94 \\
3 / 18 / 94 \\
3 / 18 / 94 \\
3 / 18 / 94\end{array}$ & $\begin{array}{l}D \\
D \\
L \\
\text { P } \\
\text { L } \\
\text { L } \\
\text { L } \\
\text { L } \\
\text { E } \\
\text { P }\end{array}$ & $\begin{array}{l}0 \\
0 \\
0 \\
.03 \\
0 \\
0 \\
0 \\
0 \\
.01 \\
2.01\end{array}$ & $\begin{array}{l}-- \\
-- \\
5.9 \\
7 \\
5.8 \\
5.6 \\
5.5 \\
6.4 \\
8 \\
8.4\end{array}$ & $\begin{array}{r}-- \\
-- \\
30 \\
44 \\
32 \\
25 \\
37 \\
63 \\
294 \\
428\end{array}$ & $\begin{array}{l}-. \\
\ddot{-} \\
13.0 \\
12.0 \\
12.5 \\
12.0 \\
12.5 \\
11.5 \\
12.5 \\
9.0\end{array}$ & $\begin{array}{l}-- \\
-- \\
8.6 \\
-- \\
-- \\
8.1 \\
4.8 \\
9.8 \\
9.7 \\
--\end{array}$ \\
\hline $\begin{array}{l}4115 \\
4120 \\
4125 \\
4400 \\
4405 \\
4410 \\
4415 \\
4420 \\
4425 \\
4430\end{array}$ & $\begin{array}{l}\text { Spring } \\
\text { Seep } \\
\text { Stream } \\
\text { Stream } \\
\text { Stream } \\
\text { Stream } \\
\text { Seep } \\
\text { Spring } \\
\text { Seep } \\
\text { Stream }\end{array}$ & $\begin{array}{l}3 / 18 / 94 \\
3 / 18 / 94 \\
3 / 18 / 94 \\
3 / 18 / 94 \\
3 / 18 / 94 \\
3 / 18 / 94 \\
3 / 18 / 94 \\
3 / 18 / 94 \\
3 / 18 / 94 \\
3 / 18 / 94\end{array}$ & $\begin{array}{l}\mathbf{P} \\
\mathbf{D} \\
\mathbf{D} \\
\mathbf{R} \\
\mathbf{P} \\
\mathbf{P} \\
\mathbf{L} \\
\mathbf{E} \\
\mathbf{L} \\
\mathbf{P}\end{array}$ & $\begin{array}{l}.44 \\
0 \\
0 \\
1.06 \\
.09 \\
.02 \\
0 \\
.01 \\
0 \\
.08\end{array}$ & $\begin{array}{l}7.4 \\
-- \\
- \\
7.7 \\
7.4 \\
6.8 \\
7.1 \\
7.8 \\
7.4 \\
7.8\end{array}$ & $\begin{array}{r}351 \\
-. \\
- \\
466 \\
321 \\
397 \\
255 \\
420 \\
321 \\
300\end{array}$ & $\begin{array}{r}12.0 \\
-- \\
- \\
9.5 \\
9.5 \\
11.5 \\
17.0 \\
13.0 \\
9.0 \\
9.0\end{array}$ & $\begin{array}{l}5.8 \\
-- \\
-- \\
-- \\
-- \\
-- \\
6 \\
5.9 \\
2.1 \\
--\end{array}$ \\
\hline $\begin{array}{l}4435 \\
4440 \\
4445 \\
4450 \\
4455 \\
4472 \\
4473 \\
4475 \\
4480 \\
4485\end{array}$ & $\begin{array}{l}\text { Stream } \\
\text { Stream } \\
\text { Seep } \\
\text { Stream } \\
\text { Stream } \\
\text { Seep } \\
\text { Spring } \\
\text { Stream } \\
\text { Stream } \\
\text { Stream }\end{array}$ & $\begin{array}{l}3 / 18 / 94 \\
3 / 18 / 94 \\
3 / 18 / 94 \\
3 / 18 / 94 \\
3 / 18 / 94 \\
3 / 18 / 94 \\
3 / 18 / 94 \\
3 / 18 / 94 \\
3 / 18 / 94 \\
3 / 18 / 94\end{array}$ & $\begin{array}{l}P \\
E \\
D \\
E \\
P \\
L \\
E \\
-- \\
P \\
V\end{array}$ & $\begin{array}{l}.04 \\
.01 \\
0 \\
.01 \\
.03 \\
0 \\
.01 \\
-.04 \\
.04\end{array}$ & $\begin{array}{l}7.6 \\
7.7 \\
-- \\
7.8 \\
8.2 \\
6.7 \\
6.6 \\
7.9 \\
7.6 \\
7.6\end{array}$ & $\begin{array}{r}709 \\
908 \\
-- \\
388 \\
1,350 \\
48 \\
38 \\
170 \\
109 \\
115\end{array}$ & $\begin{array}{r}9.0 \\
10.5 \\
-- \\
5.0 \\
8.5 \\
17.0 \\
11.0 \\
7.5 \\
8.0 \\
8.5\end{array}$ & $\begin{array}{l}-- \\
-- \\
-- \\
-- \\
-- \\
10.8 \\
-- \\
--\end{array}$ \\
\hline $\begin{array}{l}4490 \\
4495 \\
4500 \\
4505 \\
4510 \\
4515 \\
4520 \\
4525 \\
4530 \\
4535\end{array}$ & $\begin{array}{l}\text { Stream } \\
\text { Seep } \\
\text { Stream } \\
\text { Seep } \\
\text { Seep } \\
\text { Spring } \\
\text { Stream } \\
\text { Stream } \\
\text { Stream } \\
\text { Stream }\end{array}$ & $\begin{array}{l}3 / 18 / 94 \\
3 / 18 / 94 \\
3 / 18 / 94 \\
3 / 18 / 94 \\
3 / 18 / 94 \\
3 / 18 / 94 \\
3 / 18 / 94 \\
3 / 18 / 94 \\
3 / 18 / 94 \\
3 / 18 / 94\end{array}$ & $\begin{array}{l}\mathbf{E} \\
\mathbf{L} \\
\mathbf{P} \\
\mathbf{L} \\
\mathbf{E} \\
\mathbf{P} \\
\mathbf{P} \\
\mathbf{P} \\
\mathbf{P} \\
\mathbf{P}\end{array}$ & $\begin{array}{l}.01 \\
0 \\
.02 \\
.01 \\
.01 \\
.89 \\
.8 \\
.93 \\
.06\end{array}$ & $\begin{array}{l}7.6 \\
7 \\
7.3 \\
6.3 \\
6.7 \\
6.9 \\
8.1 \\
8.1 \\
7.9 \\
8.4\end{array}$ & $\begin{array}{r}323 \\
133 \\
60 \\
32 \\
78 \\
31 \\
507 \\
508 \\
513 \\
304\end{array}$ & $\begin{array}{r}8.0 \\
12.0 \\
12.0 \\
16.0 \\
10.0 \\
12.0 \\
14.5 \\
14.5 \\
14.0 \\
11.5\end{array}$ & $\begin{array}{l}-- \\
- \\
- \\
7.7 \\
1.4 \\
9.5 \\
-- \\
-- \\
-- \\
-\end{array}$ \\
\hline $\begin{array}{l}4540 \\
4545 \\
4580 \\
4585 \\
4590 \\
4595 \\
4600 \\
4605 \\
4610 \\
4615\end{array}$ & $\begin{array}{l}\text { Stream } \\
\text { Stream } \\
\text { Stream } \\
\text { Stream } \\
\text { Stream } \\
\text { Stream } \\
\text { Stream } \\
\text { Seep } \\
\text { Stream } \\
\text { Seep }\end{array}$ & $\begin{array}{l}3 / 18 / 94 \\
3 / 18 / 94 \\
3 / 18 / 94 \\
3 / 18 / 94 \\
3 / 18 / 94 \\
3 / 18 / 94 \\
3 / 18 / 94 \\
3 / 18 / 94 \\
3 / 18 / 94 \\
3 / 18 / 94\end{array}$ & $\begin{array}{l}\mathbf{P} \\
\mathbf{P} \\
\mathbf{P} \\
\mathbf{D} \\
\mathrm{D} \\
\mathbf{P} \\
\mathbf{D} \\
\mathbf{D} \\
\mathbf{P} \\
\mathbf{D}\end{array}$ & $\begin{array}{l}.07 \\
.1 \\
.07 \\
0 \\
0 \\
.06 \\
0 \\
0 \\
.02\end{array}$ & $\begin{array}{l}8.3 \\
8 \\
7.2 \\
-- \\
\overline{6} \\
-- \\
-\overline{7.6} \\
--\end{array}$ & $\begin{array}{r}307 \\
312 \\
190 \\
-- \\
-- \\
464 \\
-- \\
-- \\
156 \\
--\end{array}$ & $\begin{array}{l}12.5 \\
13.0 \\
16.0 \\
-- \\
-- \\
13.0 \\
-- \\
-- \\
14.5 \\
--\end{array}$ & $\begin{array}{l}-- \\
-- \\
-- \\
-- \\
-- \\
-- \\
-- \\
-- \\
-- \\
--\end{array}$ \\
\hline
\end{tabular}


Table 3. Discharge and water-quality data for the high base flow seepage investigation at Bear Creek Valley, Oak Ridge, Tennessee, March 14 through March 19, 1994-Continued

\begin{tabular}{|c|c|c|c|c|c|c|c|c|}
\hline $\begin{array}{c}\text { Site } \\
\text { number }\end{array}$ & $\begin{array}{l}\text { Type of } \\
\text { site }\end{array}$ & $\begin{array}{c}\text { Date } \\
\text { sampled } \\
\text { (month/ } \\
\text { day/year) }\end{array}$ & Method & $\begin{array}{l}\text { Flow } \\
\left(\mathrm{ft}^{3} / \mathrm{s}\right)\end{array}$ & $\mathbf{p H}$ & $\begin{array}{c}\text { Specific } \\
\text { conduc- } \\
\text { tance } \\
(\mu \mathrm{S} / \mathrm{cm})\end{array}$ & $\begin{array}{c}\text { Temper- } \\
\text { ature } \\
\left({ }^{\circ} \mathrm{C}\right)\end{array}$ & $\begin{array}{c}\text { Dis- } \\
\text { solved } \\
\text { oxygen } \\
\text { (mgll) }\end{array}$ \\
\hline $\begin{array}{l}4620 \\
4630 \\
5005 \\
5010 \\
5015 \\
5020 \\
5025 \\
5030 \\
5035 \\
5040\end{array}$ & $\begin{array}{l}\text { Spring } \\
\text { Stream } \\
\text { Stream } \\
\text { Stream } \\
\text { Stream } \\
\text { Stream } \\
\text { Seep } \\
\text { Stream } \\
\text { Stream } \\
\text { Seep }\end{array}$ & $\begin{array}{l}3 / 18 / 94 \\
3 / 18 / 94 \\
3 / 14 / 94 \\
3 / 14 / 94 \\
3 / 14 / 94 \\
3 / 14 / 94 \\
3 / 14 / 94 \\
3 / 14 / 94 \\
3 / 14 / 94 \\
3 / 14 / 94\end{array}$ & $\begin{array}{l}F \\
P \\
P \\
P \\
P \\
P \\
V \\
P \\
P \\
L\end{array}$ & $\begin{array}{l}.02 \\
.79 \\
3.28 \\
.03 \\
.04 \\
.03 \\
.01 \\
3.11 \\
.08 \\
0\end{array}$ & $\begin{array}{l}7.4 \\
8.2 \\
8.4 \\
8.1 \\
8 \\
6.7 \\
- \\
8.2 \\
7.9 \\
7.4\end{array}$ & $\begin{array}{r}93 \\
526 \\
348 \\
125 \\
87 \\
41 \\
49 \\
325 \\
120 \\
366\end{array}$ & $\begin{array}{l}12.5 \\
14.5 \\
12.5 \\
12.0 \\
12.0 \\
10.0 \\
12.0 \\
13.0 \\
11.0 \\
11.0\end{array}$ & $\begin{array}{l}-- \\
-- \\
9 \\
9.5 \\
7.1 \\
-- \\
-- \\
-- \\
4\end{array}$ \\
\hline $\begin{array}{l}5045 \\
5050 \\
5055 \\
5060 \\
5065 \\
5070 \\
5075 \\
5080 \\
5085 \\
5090\end{array}$ & $\begin{array}{l}\text { Stream } \\
\text { Stream } \\
\text { Stream } \\
\text { Seep } \\
\text { Seep } \\
\text { Seep } \\
\text { Stream } \\
\text { Seep } \\
\text { Seep } \\
\text { Stream }\end{array}$ & $\begin{array}{l}3 / 14 / 94 \\
3 / 14 / 94 \\
3 / 14 / 94 \\
3 / 14 / 94 \\
3 / 14 / 94 \\
3 / 14 / 94 \\
3 / 14 / 94 \\
3 / 14 / 94 \\
3 / 14 / 94 \\
3 / 14 / 94\end{array}$ & $\begin{array}{l}P \\
P \\
V \\
E \\
L \\
L \\
P \\
V \\
E \\
\text { L }\end{array}$ & $\begin{array}{l}.06 \\
.06 \\
.01 \\
.01 \\
0 \\
0 \\
.04 \\
.01 \\
.01 \\
0\end{array}$ & $\begin{array}{l}7.9 \\
7.5 \\
7.1 \\
6.1 \\
6 \\
6.4 \\
6.8 \\
6.7 \\
6.1 \\
8\end{array}$ & $\begin{array}{r}69 \\
43 \\
25 \\
29 \\
32 \\
39 \\
45 \\
66 \\
32 \\
165\end{array}$ & $\begin{array}{l}11.0 \\
10.5 \\
11.5 \\
10.5 \\
10.4 \\
13.0 \\
12.5 \\
11.5 \\
10.0 \\
11.0\end{array}$ & $\begin{array}{l}8.2 \\
8.5 \\
7.6 \\
9.4 \\
6.6 \\
2.5 \\
8.7 \\
8.9 \\
3.2 \\
--\end{array}$ \\
\hline $\begin{array}{l}5095 \\
5100 \\
5105 \\
6005 \\
6007 \\
6010 \\
6015 \\
6020 \\
6025 \\
6030\end{array}$ & $\begin{array}{l}\text { Stream } \\
\text { Seep } \\
\text { Stream } \\
\text { Stream } \\
\text { Stream } \\
\text { Stream } \\
\text { Seep } \\
\text { Seep } \\
\text { Stream } \\
\text { Stream }\end{array}$ & $\begin{array}{l}3 / 14 / 94 \\
3 / 14 / 94 \\
3 / 14 / 94 \\
3 / 15 / 94 \\
3 / 15 / 94 \\
3 / 15 / 94 \\
3 / 14 / 94 \\
3 / 14 / 94 \\
3 / 14 / 94 \\
3 / 14 / 94\end{array}$ & $\begin{array}{l}\text { V } \\
\text { V } \\
\text { P } \\
\text { P } \\
\text { L } \\
\text { E } \\
E \\
L \\
E \\
\text { P }\end{array}$ & $\begin{array}{l}.01 \\
.01 \\
3.83 \\
.1 \\
0 \\
.01 \\
.01 \\
0 \\
0.1 \\
.08\end{array}$ & $\begin{array}{l}8.3 \\
6.4 \\
8.1 \\
7.4 \\
6.4 \\
7.5 \\
7.5 \\
6.9 \\
7 \\
6.9\end{array}$ & $\begin{array}{r}95 \\
70 \\
363 \\
115 \\
57 \\
182 \\
225 \\
95 \\
55 \\
--\end{array}$ & $\begin{array}{r}10.5 \\
10.5 \\
13.5 \\
13.0 \\
11.5 \\
8.5 \\
9.0 \\
10.0 \\
11.0 \\
--\end{array}$ & $\begin{array}{l}-- \\
5.4 \\
-- \\
-- \\
-- \\
-- \\
-- \\
-- \\
-- \\
-\end{array}$ \\
\hline $\begin{array}{l}6033 \\
6035 \\
6045 \\
6050 \\
6055 \\
6060 \\
6065 \\
6070 \\
6075 \\
6085\end{array}$ & $\begin{array}{l}\text { Seep } \\
\text { Stream } \\
\text { Seep } \\
\text { Stream } \\
\text { Seep } \\
\text { Stream } \\
\text { Stream } \\
\text { Stream } \\
\text { Stream } \\
\text { Seep }\end{array}$ & $\begin{array}{l}3 / 14 / 94 \\
3 / 14 / 94 \\
3 / 14 / 94 \\
3 / 14 / 94 \\
3 / 14 / 94 \\
3 / 14 / 94 \\
3 / 14 / 94 \\
3 / 14 / 94 \\
3 / 14 / 94 \\
3 / 14 / 94\end{array}$ & $\begin{array}{l}\mathbf{E} \\
\mathbf{V} \\
\mathbf{F} \\
\mathbf{P} \\
\mathbf{P} \\
\mathbf{P} \\
\mathbf{P} \\
\mathbf{P} \\
\mathbf{L} \\
\mathbf{L}\end{array}$ & $\begin{array}{l}.01 \\
.03 \\
.02 \\
.03 \\
.01 \\
3.33 \\
3.64 \\
.16 \\
0 \\
0\end{array}$ & $\begin{array}{l}5.2 \\
7.5 \\
6.4 \\
7.8 \\
6.7 \\
8.3 \\
8.3 \\
7.6 \\
6.4 \\
7.3\end{array}$ & $\begin{array}{r}29 \\
48 \\
40 \\
32 \\
39 \\
340 \\
252 \\
81 \\
51 \\
125\end{array}$ & $\begin{array}{r}9.5 \\
10.0 \\
10.5 \\
9.5 \\
10.0 \\
14.0 \\
13.0 \\
12.0 \\
8.0 \\
8.5\end{array}$ & $\begin{array}{l}- \\
- \\
- \\
- \\
- \\
- \\
- \\
- \\
- \\
-\end{array}$ \\
\hline $\begin{array}{l}6090 \\
6095 \\
6100 \\
6105 \\
6110 \\
6115 \\
6120 \\
7005 \\
7010 \\
7015\end{array}$ & $\begin{array}{l}\text { Stream } \\
\text { Seep } \\
\text { Stream } \\
\text { Seep } \\
\text { Seep } \\
\text { Stream } \\
\text { Spring } \\
\text { Stream } \\
\text { Stream } \\
\text { Stream }\end{array}$ & $\begin{array}{l}3 / 14 / 94 \\
3 / 14 / 94 \\
3 / 15 / 94 \\
3 / 15 / 94 \\
3 / 15 / 94 \\
3 / 15 / 94 \\
3 / 15 / 94 \\
3 / 14 / 94 \\
3 / 14 / 94 \\
3 / 14 / 94\end{array}$ & $\begin{array}{l}\text { L } \\
\text { L } \\
\text { P } \\
\text { L } \\
D \\
P \\
\text { V } \\
P \\
P \\
P\end{array}$ & $\begin{array}{l}0 \\
0 \\
.14 \\
0 \\
0 \\
.05 \\
.01 \\
3.04 \\
.08 \\
.33\end{array}$ & $\begin{array}{l}6.9 \\
7.3 \\
-- \\
6.8 \\
7.6 \\
8.4 \\
8.1 \\
7.4\end{array}$ & $\begin{array}{r}26 \\
- \\
87 . \\
-- \\
- \\
25 \\
90 \\
348 \\
95 \\
95\end{array}$ & $\begin{array}{c}8.0 \\
-\overline{13.0} \\
-- \\
- \\
12.0 \\
11.5 \\
13.0 \\
13.0 \\
12.0\end{array}$ & $\begin{array}{l}-- \\
-- \\
-- \\
-- \\
- \\
10.5 \\
-- \\
-- \\
--\end{array}$ \\
\hline
\end{tabular}


Table 3. Discharge and water-quality data for the high base flow seepage investigation at Bear Creek Valley, Oak Ridge, Tennessee, March 14 through March 19, 1994-Continued

\begin{tabular}{|c|c|c|c|c|c|c|c|c|}
\hline $\begin{array}{c}\text { Site } \\
\text { number }\end{array}$ & $\begin{array}{l}\text { Type of } \\
\text { site }\end{array}$ & $\begin{array}{c}\text { Date } \\
\text { sampled } \\
\text { (month/ } \\
\text { day/year) }\end{array}$ & Method & $\begin{array}{l}\text { Flow } \\
\left(\mathrm{ft}^{3} / \mathrm{s}\right)\end{array}$ & pH & $\begin{array}{c}\text { Specific } \\
\text { conduc- } \\
\text { tance } \\
(\mu \mathrm{S} / \mathrm{cm})\end{array}$ & $\begin{array}{c}\text { Temper- } \\
\text { ature } \\
\left({ }^{\circ} \mathrm{C}\right)\end{array}$ & $\begin{array}{c}\text { Dis- } \\
\text { solved } \\
\text { oxygen } \\
\text { (mg/l) }\end{array}$ \\
\hline $\begin{array}{l}7020 \\
7025 \\
7030 \\
7035 \\
7040 \\
7045 \\
7050 \\
7055 \\
7060 \\
8005\end{array}$ & $\begin{array}{l}\text { Spring } \\
\text { Stream } \\
\text { Stream } \\
\text { Stream } \\
\text { Wetland } \\
\text { Stream } \\
\text { Seep } \\
\text { Stream } \\
\text { Stream } \\
\text { Stream }\end{array}$ & $\begin{array}{l}3 / 14 / 94 \\
3 / 14 / 94 \\
3 / 14 / 94 \\
3 / 14 / 94 \\
3 / 14 / 94 \\
3 / 14 / 94 \\
3 / 14 / 94 \\
3 / 14 / 94 \\
3 / 14 / 94 \\
3 / 15 / 94\end{array}$ & $\begin{array}{l}P \\
P \\
P \\
E \\
D \\
E \\
E \\
D \\
P \\
P\end{array}$ & $\begin{array}{l}.05 \\
.16 \\
.13 \\
.04 \\
0 \\
.01 \\
.01 \\
0 \\
3.67 \\
.27\end{array}$ & $\begin{array}{l}7 \\
7.4 \\
6.7 \\
5.3 \\
-- \\
5 \\
7.7 \\
-- \\
8.4 \\
7.3\end{array}$ & $\begin{array}{r}165 \\
60 \\
39 \\
16 \\
-- \\
16 \\
230 \\
-- \\
349 \\
77\end{array}$ & $\begin{array}{c}12.5 \\
14.0 \\
12.5 \\
11.0 \\
-- \\
11.0 \\
18.5 \\
-\overline{13.0} \\
14.5\end{array}$ & $\begin{array}{l}8.7 \\
-- \\
-- \\
-- \\
-- \\
7.5 \\
9 \\
--\end{array}$ \\
\hline $\begin{array}{l}8010 \\
8015 \\
8020 \\
8025 \\
8030 \\
8035 \\
8040 \\
8045 \\
8050 \\
8055\end{array}$ & $\begin{array}{l}\text { Seep } \\
\text { Seep } \\
\text { Stream } \\
\text { Stream } \\
\text { Stream } \\
\text { Stream } \\
\text { Seep } \\
\text { Seep } \\
\text { Seep } \\
\text { Stream }\end{array}$ & $\begin{array}{l}3 / 15 / 94 \\
3 / 15 / 94 \\
3 / 15 / 94 \\
3 / 15 / 94 \\
3 / 15 / 94 \\
3 / 15 / 94 \\
3 / 15 / 94 \\
3 / 15 / 94 \\
3 / 15 / 94 \\
3 / 17 / 94\end{array}$ & $\begin{array}{l}\text { D } \\
\text { D } \\
\text { P } \\
\text { L } \\
\text { L } \\
\text { D } \\
\text { D } \\
\text { D } \\
\text { D } \\
\text { P }\end{array}$ & $\begin{array}{l}0 \\
0 \\
.24 \\
0 \\
0 \\
0 \\
0 \\
0 \\
0 \\
.16\end{array}$ & $\begin{array}{l}-- \\
\ddot{7.2} \\
6.6 \\
5.6 \\
-- \\
-- \\
-- \\
-- \\
6.5\end{array}$ & $\begin{array}{c}-- \\
-- \\
51 \\
43 \\
28 \\
-- \\
- \\
- \\
-- \\
68\end{array}$ & $\begin{array}{c}-- \\
-- \\
13.5 \\
10.0 \\
9.0 \\
-- \\
- \\
-- \\
- \\
10.0\end{array}$ & $\begin{array}{l}-- \\
- \\
-- \\
- \\
- \\
- \\
- \\
-- \\
- \\
-\end{array}$ \\
\hline $\begin{array}{l}8060 \\
8062 \\
8063 \\
8065 \\
8070 \\
8075 \\
8080 \\
8085 \\
8090 \\
8092\end{array}$ & $\begin{array}{l}\text { Seep } \\
\text { Stream } \\
\text { Seep } \\
\text { Stream } \\
\text { Stream } \\
\text { Seep } \\
\text { Stream } \\
\text { Seep } \\
\text { Stream } \\
\text { Seep }\end{array}$ & $\begin{array}{l}3 / 17 / 94 \\
3 / 17 / 94 \\
3 / 17 / 94 \\
3 / 17 / 94 \\
3 / 17 / 94 \\
3 / 17 / 94 \\
3 / 17 / 94 \\
3 / 17 / 94 \\
3 / 17 / 94 \\
3 / 17 / 94\end{array}$ & $\begin{array}{l}\text { L } \\
\text { L } \\
\text { L } \\
\text { E } \\
\text { L } \\
D \\
E \\
E \\
P \\
D\end{array}$ & $\begin{array}{l}0 \\
0 \\
0 \\
.08 \\
0 \\
0 \\
.01 \\
.01 \\
.23 \\
0^{.01}\end{array}$ & $\begin{array}{l}5.1 \\
7.1 \\
-- \\
6.2 \\
6 \\
- \\
6.1 \\
5.8 \\
6.8 \\
--\end{array}$ & $\begin{array}{r}29 \\
251 \\
-- \\
39 \\
42 \\
-- \\
43 \\
64 \\
47 \\
--\end{array}$ & $\begin{array}{c}9.5 \\
8.0 \\
-- \\
10.0 \\
8.5 \\
-- \\
9.0 \\
11.0 \\
10.0 \\
--\end{array}$ & $\begin{array}{l}-- \\
-- \\
-- \\
-- \\
-- \\
-- \\
-- \\
8.5 \\
-- \\
--\end{array}$ \\
\hline $\begin{array}{l}8095 \\
8100 \\
8103 \\
8105 \\
8110 \\
8115 \\
8120 \\
8125 \\
8130 \\
8135\end{array}$ & $\begin{array}{l}\text { Stream } \\
\text { Seep } \\
\text { Stream } \\
\text { Stream } \\
\text { Seep } \\
\text { Stream } \\
\text { Seep } \\
\text { Stream } \\
\text { Seep } \\
\text { Stream }\end{array}$ & $\begin{array}{l}3 / 17 / 94 \\
3 / 17 / 94 \\
3 / 17 / 94 \\
3 / 17 / 94 \\
3 / 17 / 94 \\
3 / 17 / 94 \\
3 / 17 / 94 \\
3 / 17 / 94 \\
3 / 17 / 94 \\
3 / 16 / 94\end{array}$ & $\begin{array}{l}\text { L } \\
\text { L } \\
\text { P } \\
\text { L } \\
\text { L } \\
\text { L } \\
\text { L } \\
\text { E } \\
\text { E } \\
\text { D }\end{array}$ & $\begin{array}{l}0 \\
0 \\
.08 \\
0 \\
0 \\
0 \\
0 \\
.04 \\
.04 \\
0\end{array}$ & $\begin{array}{l}6.9 \\
7.3 \\
6.3 \\
6.2 \\
5.8 \\
5.7 \\
5.8 \\
5.9 \\
6 \\
--\end{array}$ & $\begin{array}{l}77 \\
89 \\
42 \\
55 \\
55 \\
48 \\
38 \\
47 \\
45 \\
--\end{array}$ & $\begin{array}{r}6.0 \\
5.5 \\
6.0 \\
9.0 \\
11.0 \\
8.5 \\
10.5 \\
7.0 \\
10.5 \\
--\end{array}$ & $\begin{array}{l}-- \\
10.9 \\
- \\
- \\
7 \\
- \\
7.4 \\
-- \\
- \\
--\end{array}$ \\
\hline $\begin{array}{l}8140 \\
8145 \\
8150 \\
8200 \\
8205 \\
8210 \\
8220 \\
8225 \\
9005 \\
9010\end{array}$ & $\begin{array}{l}\text { Seep } \\
\text { Seep } \\
\text { Seep } \\
\text { Seep } \\
\text { Seep } \\
\text { Stream } \\
\text { Seep } \\
\text { Seep } \\
\text { Stream } \\
\text { Stream }\end{array}$ & $\begin{array}{l}3 / 16 / 94 \\
3 / 16 / 94 \\
3 / 16 / 94 \\
3 / 16 / 94 \\
3 / 16 / 94 \\
3 / 16 / 94 \\
3 / 16 / 94 \\
3 / 16 / 94 \\
3 / 16 / 94 \\
3 / 16 / 94\end{array}$ & $\begin{array}{l}\text { D } \\
\text { D } \\
\text { L } \\
\text { D } \\
\text { D } \\
\text { D } \\
\text { D } \\
\text { L } \\
\text { P } \\
\text { P }\end{array}$ & $\begin{array}{l}0 \\
0 \\
0 \\
0 \\
0 \\
0 \\
0 \\
0 \\
4.24 \\
.3\end{array}$ & $\begin{array}{l}-\overline{-} \\
\overline{5.3} \\
-- \\
-- \\
-- \\
- \\
6.7 \\
7.7 \\
8.2\end{array}$ & $\begin{array}{r}-- \\
38 \\
-- \\
-- \\
- \\
- \\
42 \\
208 \\
193\end{array}$ & $\begin{array}{l}-- \\
-- \\
8.0 \\
-- \\
-- \\
-- \\
-- \\
9.5 \\
7.5 \\
13.5\end{array}$ & $\begin{array}{l}-- \\
-- \\
9 \\
-- \\
-- \\
-- \\
- \\
-- \\
-- \\
--\end{array}$ \\
\hline
\end{tabular}


Table 3. Discharge and water-quality data for the high base flow seepage investigation at Bear Creek Valley, Oak Ridge, Tennessee, March 14 through March 19, 1994-Continued

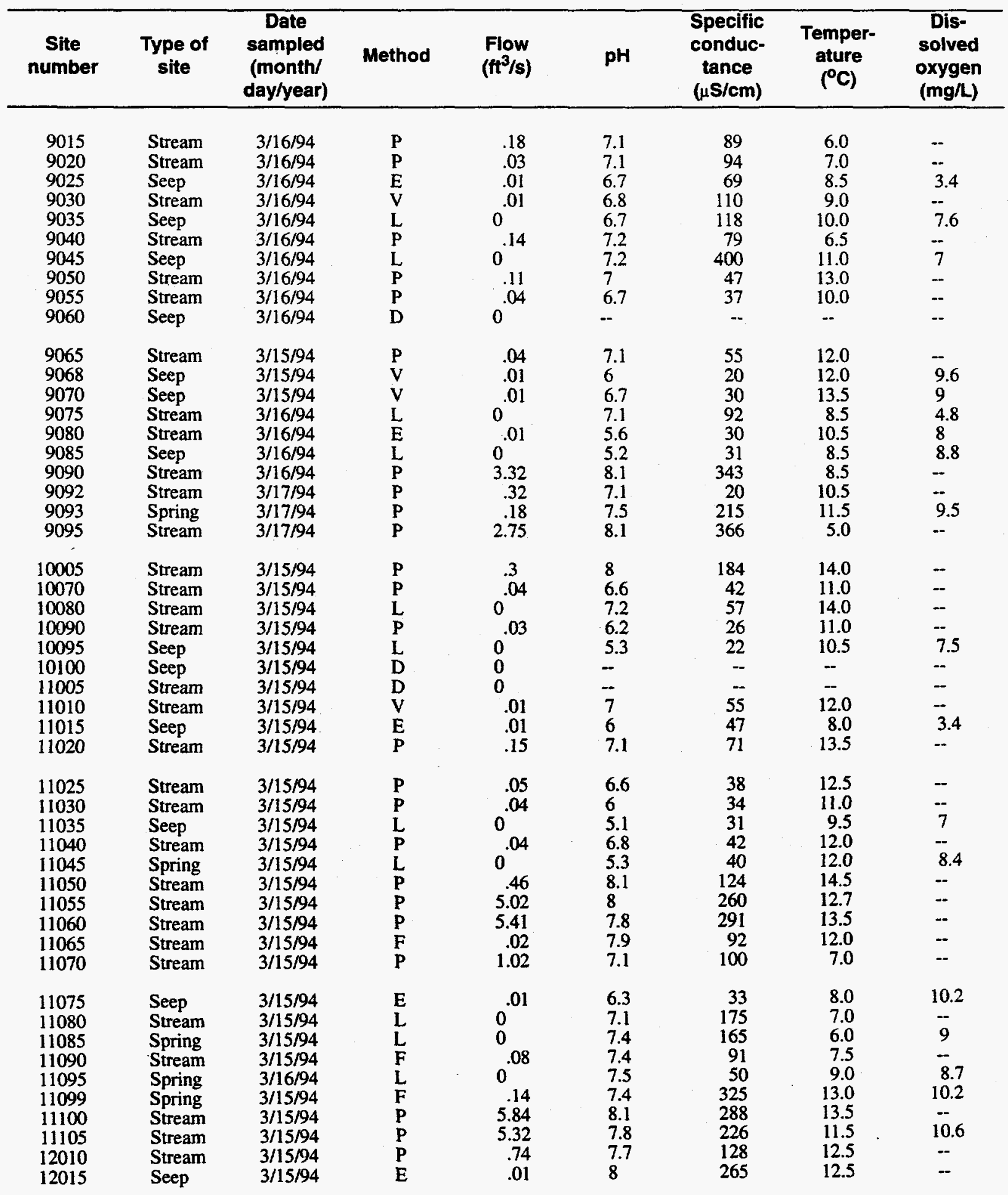


Table 3. Discharge and water-quality data for the high base flow seepage investigation at Bear Creek Valley, Oak Ridge, Tennessee, March 14 through March 19, 1994-Continued

\begin{tabular}{|c|c|c|c|c|c|c|c|c|}
\hline $\begin{array}{c}\text { Site } \\
\text { number }\end{array}$ & $\begin{array}{l}\text { Type of } \\
\text { site }\end{array}$ & $\begin{array}{c}\text { Date } \\
\text { sampled } \\
\text { (month/ } \\
\text { day/year) }\end{array}$ & Method & $\begin{array}{l}\text { Flow } \\
\left(\mathrm{ft}^{3} / \mathrm{s}\right)\end{array}$ & pH & $\begin{array}{c}\text { Specific } \\
\text { conduc- } \\
\text { tance } \\
(\mu \mathrm{S} / \mathrm{cm})\end{array}$ & $\begin{array}{l}\text { Temper- } \\
\text { ature } \\
\left({ }^{\circ} \mathrm{C}\right)\end{array}$ & $\begin{array}{c}\text { Dis- } \\
\text { solved } \\
\text { oxygen } \\
(\mathrm{mg} / \mathrm{L})\end{array}$ \\
\hline $\begin{array}{l}12020 \\
12025 \\
12040 \\
12045 \\
12050 \\
13005 \\
13007 \\
13008 \\
13010 \\
13015\end{array}$ & $\begin{array}{l}\text { Seep } \\
\text { Spring } \\
\text { Stream } \\
\text { Stream } \\
\text { Seep } \\
\text { Stream } \\
\text { Stream } \\
\text { Seep } \\
\text { Seep } \\
\text { Seep }\end{array}$ & $\begin{array}{l}3 / 15 / 94 \\
3 / 15 / 94 \\
3 / 15 / 94 \\
3 / 15 / 94 \\
3 / 15 / 94 \\
3 / 17 / 94 \\
3 / 17 / 94 \\
3 / 17 / 94 \\
3 / 17 / 94 \\
3 / 17 / 94\end{array}$ & $\begin{array}{l}P \\
P \\
P \\
P \\
P \\
P \\
L \\
L \\
L \\
L\end{array}$ & $\begin{array}{l}.02 \\
.7 \\
.06 \\
.04 \\
.04 \\
6.89 \\
0 \\
0 \\
0 \\
0\end{array}$ & $\begin{array}{l}7.9 \\
7.1 \\
6.1 \\
8.1 \\
6.1 \\
7.7 \\
6.8 \\
6.9 \\
6.3 \\
6.4\end{array}$ & $\begin{array}{r}188 \\
118 \\
29 \\
103 \\
30 \\
260 \\
84 \\
65 \\
88 \\
32\end{array}$ & $\begin{array}{r}13.5 \\
11.5 \\
10.5 \\
12.0 \\
11.0 \\
9.5 \\
8.0 \\
8.5 \\
8.5 \\
12.0\end{array}$ & $\begin{array}{l}9.8 \\
8.9 \\
-- \\
\overline{7.2} \\
-- \\
\overline{10.8} \\
10.1 \\
10.3\end{array}$ \\
\hline $\begin{array}{l}13020 \\
13025 \\
13030 \\
13035 \\
13040 \\
13060 \\
13070 \\
13075 \\
13080 \\
13085\end{array}$ & $\begin{array}{l}\text { Spring } \\
\text { Stream } \\
\text { Stream } \\
\text { Seep } \\
\text { Seep } \\
\text { Stream } \\
\text { Stream } \\
\text { Stream } \\
\text { Seep } \\
\text { Stream }\end{array}$ & $\begin{array}{l}3 / 17 / 94 \\
3 / 179494 \\
3 / 17 / 94 \\
3 / 17 / 94 \\
3 / 17 / 94 \\
3 / 16 / 94 \\
3 / 17 / 194 \\
3 / 17 / 94 \\
3 / 17 / 94 \\
3 / 17 / 94\end{array}$ & $\begin{array}{l}\mathrm{L} \\
\mathbf{P} \\
\mathbf{E} \\
\mathrm{D} \\
\mathrm{L} \\
\mathbf{P} \\
\mathbf{P} \\
\mathrm{E} \\
\mathbf{E} \\
\mathbf{E}\end{array}$ & $\begin{array}{l}0 \\
6.2 \\
.01 \\
0 \\
0 \\
.21 \\
.1 \\
.01 \\
.01 \\
.05\end{array}$ & $\begin{array}{l}6.4 \\
7.7 \\
6.1 \\
- \\
6.8 \\
7.3 \\
7.1 \\
6.4 \\
6.1 \\
6.3\end{array}$ & $\begin{array}{r}14 \\
257 \\
53 \\
-- \\
30 \\
44 \\
47 \\
35 \\
40 \\
34\end{array}$ & $\begin{array}{r}11.5 \\
9.5 \\
8.0 \\
-. \\
10.0 \\
7.5 \\
5.5 \\
8.5 \\
10.5 \\
8.5\end{array}$ & $\begin{array}{l}9.8 \\
-- \\
- \\
- \\
7 \\
-- \\
-- \\
-- \\
--\end{array}$ \\
\hline $\begin{array}{l}13086 \\
13087 \\
13088 \\
13089 \\
13090 \\
13095 \\
13140 \\
13150 \\
13155 \\
13160\end{array}$ & $\begin{array}{l}\text { Seep } \\
\text { Stream } \\
\text { Stream } \\
\text { Seep } \\
\text { Stream } \\
\text { Seep } \\
\text { Stream } \\
\text { Seep } \\
\text { Stream } \\
\text { Stream }\end{array}$ & $\begin{array}{l}3 / 17 / 94 \\
3 / 17 / 94 \\
3 / 17 / 94 \\
3 / 17 / 94 \\
3 / 17 / 94 \\
3 / 17 / 94 \\
3 / 17 / 94 \\
3 / 17 / 94 \\
3 / 17 / 94 \\
3 / 17 / 94\end{array}$ & $\begin{array}{l}E \\
P \\
E \\
L \\
E \\
E \\
L \\
L \\
P \\
L\end{array}$ & $\begin{array}{l}- \\
.08 \\
.01 \\
0 \\
.05 \\
.01 \\
0 \\
0 \\
.07 \\
0\end{array}$ & $\begin{array}{l}5.9 \\
7.1 \\
7.5 \\
6.9 \\
6.9 \\
5.8 \\
7.7 \\
5.8 \\
7 \\
6.2\end{array}$ & $\begin{array}{r}34 \\
49 \\
131 \\
132 \\
37 \\
30 \\
70 \\
46 \\
37 \\
62\end{array}$ & $\begin{array}{r}9.0 \\
6.5 \\
7.5 \\
11.0 \\
9.5 \\
9.0 \\
8.5 \\
10.5 \\
8.5 \\
7.5\end{array}$ & $\begin{array}{l}8 \\
- \\
6.2 \\
-7 \\
\overline{7} \\
7.4 \\
--\end{array}$ \\
\hline $\begin{array}{l}13165 \\
13170 \\
13175 \\
13180 \\
13200 \\
13205 \\
13210 \\
13215 \\
13220 \\
13225\end{array}$ & $\begin{array}{l}\text { Seep } \\
\text { Stream } \\
\text { Spring } \\
\text { Stream } \\
\text { Spring } \\
\text { Stream } \\
\text { Stream } \\
\text { Seep } \\
\text { Seep } \\
\text { Stream }\end{array}$ & $\begin{array}{l}3 / 17 / 94 \\
3 / 17994 \\
3 / 17994 \\
3 / 17 / 94 \\
3 / 17 / 94 \\
3 / 17 / 94 \\
3 / 17 / 94 \\
3 / 1794 \\
3 / 17 / 94 \\
3 / 17 / 94\end{array}$ & $\begin{array}{l}\mathbf{L} \\
\mathbf{E} \\
\mathbf{E} \\
\mathbf{E} \\
\mathbf{E} \\
\mathbf{P} \\
\mathbf{P} \\
\mathbf{L} \\
\mathbf{D} \\
\mathbf{P}\end{array}$ & $\begin{array}{l}0 \\
.01 \\
.03 \\
.02 \\
.02 \\
.16 \\
.08 \\
0 \\
0 \\
.04\end{array}$ & $\begin{array}{l}5.3 \\
7.7 \\
7.2 \\
5.8 \\
6.8 \\
7.1 \\
7.5 \\
6.9 \\
7.2\end{array}$ & $\begin{array}{r}37 \\
41 \\
56 \\
31 \\
53 \\
58 \\
54 \\
171 \\
-\overline{44}\end{array}$ & $\begin{array}{c}9.5 \\
8.0 \\
10 \\
8.0 \\
10.5 \\
7.5 \\
9.0 \\
8.5 \\
-- \\
10.0\end{array}$ & $\begin{array}{l}6.2 \\
\overline{10.2} \\
-- \\
9.6 \\
-- \\
\overline{3.6} \\
-- \\
--\end{array}$ \\
\hline $\begin{array}{l}13230 \\
13235 \\
13240 \\
13245 \\
13250 \\
13255 \\
13260 \\
13265 \\
13270 \\
13275\end{array}$ & $\begin{array}{l}\text { Stream } \\
\text { Spring } \\
\text { Seep } \\
\text { Stream } \\
\text { Seep } \\
\text { Stream } \\
\text { Stream } \\
\text { Seep } \\
\text { Stream } \\
\text { Seep }\end{array}$ & $\begin{array}{l}3 / 17 / 94 \\
3 / 17 / 94 \\
3 / 17 / 94 \\
3 / 18 / 94 \\
3 / 18 / 94 \\
3 / 18 / 94 \\
3 / 18 / 94 \\
3 / 18 / 94 \\
3 / 18 / 94 \\
3 / 18 / 94\end{array}$ & $\begin{array}{l}\text { L } \\
L \\
D \\
E \\
E \\
P \\
L \\
L \\
L \\
L\end{array}$ & $\begin{array}{l}0 \\
0 \\
0 \\
.01 \\
.01 \\
.03 \\
0 \\
0 \\
0 \\
0\end{array}$ & $\begin{array}{l}7.3 \\
6.7 \\
-6.7 \\
6.5 \\
6.8 \\
6.3 \\
6.1 \\
6.2 \\
5.7\end{array}$ & $\begin{array}{r}55 \\
101 \\
- \\
37 \\
30 \\
39 \\
82 \\
74 \\
40 \\
31\end{array}$ & $\begin{array}{r}8.0 \\
12.5 \\
-7.0 \\
7.0 \\
7.0 \\
7.0 \\
7.5 \\
7.5 \\
8.5 \\
8.0\end{array}$ & $\begin{array}{l}-\overline{7.3} \\
-- \\
\ddot{4} \\
-- \\
\ddot{3} .9 \\
\ddot{3} .9\end{array}$ \\
\hline
\end{tabular}


Table 3. Discharge and water-quality data for the high base flow seepage investigation at Bear Creek Valley, Oak Ridge, Tennessee, March 14 through March 19, 1994-Continued

\begin{tabular}{|c|c|c|c|c|c|c|c|c|}
\hline $\begin{array}{c}\text { Site } \\
\text { number }\end{array}$ & $\begin{array}{l}\text { Type of } \\
\text { site }\end{array}$ & $\begin{array}{c}\text { Date } \\
\text { sampled } \\
\text { (month/ } \\
\text { day/year) }\end{array}$ & Method & $\begin{array}{l}\text { Flow } \\
\left(\mathrm{ft}^{3} / \mathrm{s}\right)\end{array}$ & pH & $\begin{array}{c}\text { Specific } \\
\text { conduc- } \\
\text { tance } \\
(\mu \mathrm{S} / \mathrm{cm})\end{array}$ & $\begin{array}{c}\text { Temper- } \\
\text { ature } \\
\left({ }^{\circ} \mathrm{C}\right)\end{array}$ & $\begin{array}{c}\text { Dis- } \\
\text { solved } \\
\text { oxygen } \\
\text { (mg/l) }\end{array}$ \\
\hline 13900 & Stream & $3 / 18 / 94$ & $\mathbf{P}$ & 6.63 & 8 & 244 & 7.5 & - \\
\hline 14005 & Stream & $3 / 19 / 94$ & $\mathbf{P}$ & 5.78 & 7.9 & 282 & 9.0 & -. \\
\hline 14010 & Stream & $3 / 19 / 94$ & $\mathbf{P}$ & 5.49 & 7.9 & 281 & 9.5 & - \\
\hline 14015 & Stream & $3 / 19 / 94$ & D & 0 & -- & -. & -- & -. \\
\hline 14025 & Seep & $3 / 19 / 94$ & D & 0 & -- & -- & -- & -- \\
\hline 14035 & Stream & $3 / 18 / 94$ & $\mathbf{P}$ & .08 & 7.7 & 144 & 6.0 & - \\
\hline 14038 & Stream & $3 / 18 / 94$ & L & 0 & -- & - & -- & - \\
\hline 14040 & Stream & $3 / 18 / 94$ & $\overrightarrow{\mathbf{P}}$ & .18 & 7.6 & 134 & 6.0 & -- \\
\hline 14045 & Stream & $3 / 18 / 94$ & V & .01 & 6.9 & 63 & 5.5 & - \\
\hline 14050 & Stream & $3 / 18 / 94$ & V & .01 & 7 & 70 & 5.5 & -- \\
\hline 14060 & Seep & $3 / 18 / 94$ & $\mathbf{E}$ & .01 & 6.6 & 86 & 10.0 & 5.1 \\
\hline 14065 & Wetland & $3 / 18 / 94$ & - & 0 & 6.8 & 102 & 13.0 & 3.8 \\
\hline 14070 & Stream & $3 / 18 / 94$ & $\mathbf{P}$ & .11 & 7.5 & 90 & 11.5 & - \\
\hline 14075 & Stream & $3 / 18 / 94$ & $\mathbf{P}$ & .13 & 7.9 & 108 & 12.5 & -- \\
\hline 14080 & Stream & $3 / 18 / 94$ & $\mathbf{E}$ & .01 & 6.3 & 30 & 10.5 & - \\
\hline 14085 & Stream & $3 / 18 / 94$ & $\mathbf{L}$ & 0 & 5.4 & 33 & 10.5 & - \\
\hline 14090 & Seep & $3 / 18 / 94$ & D & 0 & -. & - & -- & - \\
\hline 14095 & Stream & $3 / 19 / 94$ & D & 0 & -- & -. & -- & - \\
\hline 14097 & Stream & $3 / 19 / 94$ & $\mathbf{P}$ & 5.25 & 7.8 & 283 & 9.0 & - \\
\hline 14100 & Seep & $3 / 18 / 94$ & $\mathbf{L}$ & 0 & 7.8 & 252 & 12.0 & 6.4 \\
\hline 14105 & Stream & $3 / 18 / 94$ & $\mathbf{R}$ & 6.67 & 7.6 & 280 & 12.5 & - \\
\hline 14110 & Spring & $3 / 18 / 94$ & $\mathbf{P}$ & 1 & 7.7 & 226 & 12.0 & 8.5 \\
\hline 14115 & Spring & $3 / 18 / 94$ & $\mathbf{P}$ & .3 & 7.4 & 243 & 12.5 & 7.9 \\
\hline 14120 & Seep & $3 / 18 / 94$ & E & .01 & 7.1 & 146 & 11.5 & 5.4 \\
\hline 14125 & Stream & $3 / 18 / 94$ & $\overline{\mathbf{P}}$ & 4.26 & 8.3 & 291 & 13.0 & $\because 0$ \\
\hline 14130 & Seep & $3 / 18 / 94$ & E & .01 & 6.6 & 96 & 8.5 & 1.2 \\
\hline 14133 & Stream & $3 / 18 / 94$ & $\mathbf{P}$ & 3.46 & 8.3 & 312 & 8.0 & - \\
\hline 14135 & Stream & $3 / 18 / 94$ & $\mathbf{P}$ & 3.28 & 8.3 & 315 & 8.5 & -- \\
\hline 14190 & Seep & $3 / 19 / 94$ & $\mathbf{L}$ & 0 & 6 & 45 & 10.0 & 8.4 \\
\hline 14195 & Seep & $3 / 19 / 94$ & $\mathbf{L}$ & 0 & 6.8 & 123 & 8.5 & 6.1 \\
\hline 14200 & Seep & $3 / 19 / 94$ & $\mathbf{L}$ & 0 & 7.3 & 36 & 9.0 & 10.6 \\
\hline 15005 & Stream & $3 / 19 / 94$ & $\mathbf{P}$ & .21 & 8.1 & 166 & 10.0 & -- \\
\hline 15010 & Stream & $3 / 19 / 94$ & $\mathbf{P}$ & .26 & 7.8 & 177 & 10.0 & -- \\
\hline 15015 & Stream & $3 / 19 / 94$ & L & 0 & 7.1 & 149 & 9.5 & -- \\
\hline 15020 & Seep & $3 / 19 / 94$ & $L$ & 0 & 7.5 & 214 & 11.0 & -- \\
\hline 15025 & Seep & $3 / 18 / 94$ & D & 0 & -- & -- & -- & -- \\
\hline 15030 & Seep & $3 / 18 / 94$ & D & 0 & - & $\ddot{0}$ & $\ddot{n}$ & -- \\
\hline 15035 & Stream & $3 / 19 / 94$ & $\bar{E}$ & .01 & 7.2 & 155 & 10.5 & -- \\
\hline 15040 & Stream & $3 / 18 / 94$ & $\mathbf{P}$ & .06 & 7.5 & 272 & 14.0 & 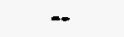 \\
\hline 15045 & Stream & $3 / 18 / 94$ & $\mathbf{E}$ & .01 & 7.5 & 122 & 12.5 & - \\
\hline 15055 & Stream & $3 / 18 / 94$ & $\mathbf{E}$ & .15 & 7 & 98 & 11.0 & -- \\
\hline 15056 & Stream & $3 / 18 / 94$ & $\mathbf{P}$ & .02 & 7 & 143 & 11.0 & - \\
\hline 15065 & Seep & $3 / 18 / 94$ & $\mathbf{L}$ & 0 & 5.7 & 57 & 13.0 & 5.5 \\
\hline 15070 & Seep & $3 / 18 / 94$ & D & 0 & - & - & - & - \\
\hline 15075 & Seep & $3 / 18 / 94$ & $\mathrm{E}$ & .01 & 6.9 & 71 & 10.5 & 7 \\
\hline 15080 & Seep & $3 / 18 / 94$ & $\mathbf{L}$ & 0 & 6.7 & 179 & 11.0 & 5 \\
\hline 15081 & Seep & $3 / 18 / 94$ & E & .01 & 6.3 & 69 & 10.5 & 6.8 \\
\hline 15085 & Seep & $3 / 18 / 94$ & $\bar{L}$ & 0 & 7.4 & 284 & 13.5 & 8.3 \\
\hline 16005 & Seep & $3 / 18 / 94$ & $\bar{D}$ & 0 & - & - & $\because$ & $\cdots$ \\
\hline 16010 & Stream & $3 / 19 / 94$ & $\mathbf{P}$ & .1 & 6.7 & 121 & 6.0 & $\cdots$ \\
\hline
\end{tabular}


Table 3. Discharge and water-quality data for the high base flow seepage investigation at Bear Creek Valley, Oak Ridge, Tennessee, March 14 through March 19, 1994-Continued

\begin{tabular}{|c|c|c|c|c|c|c|c|c|}
\hline $\begin{array}{c}\text { Site } \\
\text { number }\end{array}$ & $\begin{array}{l}\text { Type of } \\
\text { site }\end{array}$ & $\begin{array}{c}\text { Date } \\
\text { sampled } \\
\text { (month/ } \\
\text { day/year) }\end{array}$ & Method & $\begin{array}{c}\text { Flow } \\
\left(\mathrm{ft}^{3} / \mathrm{s}\right)\end{array}$ & pH & $\begin{array}{c}\text { Specific } \\
\text { conduc- } \\
\text { tance } \\
(\mu \mathrm{S} / \mathrm{cm})\end{array}$ & $\begin{array}{c}\text { Temper- } \\
\text { ature } \\
\left({ }^{\circ} \mathrm{C}\right)\end{array}$ & $\begin{array}{c}\text { Dis- } \\
\text { solved } \\
\text { oxygen } \\
\text { (mg/L) }\end{array}$ \\
\hline $\begin{array}{l}16015 \\
16020 \\
16025 \\
16030 \\
16035 \\
16040 \\
16045 \\
16050 \\
16055 \\
16060\end{array}$ & $\begin{array}{l}\text { Seep } \\
\text { Seep } \\
\text { Stream } \\
\text { Seep } \\
\text { Stream } \\
\text { Seep } \\
\text { Stream } \\
\text { Stream } \\
\text { Spring } \\
\text { Seep }\end{array}$ & $\begin{array}{l}3 / 19 / 94 \\
3 / 19 / 94 \\
3 / 19 / 94 \\
3 / 19 / 94 \\
3 / 19 / 94 \\
3 / 19 / 94 \\
3 / 19 / 94 \\
3 / 19 / 94 \\
3 / 19 / 94 \\
3 / 19 / 94\end{array}$ & $\begin{array}{l}\text { L } \\
\text { L } \\
\text { V } \\
\text { L } \\
P \\
\text { L } \\
P \\
E \\
E \\
L\end{array}$ & $\begin{array}{l}0 \\
0 \\
.01 \\
0 \\
.06 \\
0 \\
.04 \\
.01 \\
.02 \\
0\end{array}$ & $\begin{array}{l}6.8 \\
7.1 \\
7.4 \\
6.7 \\
7.6 \\
6.7 \\
7.2 \\
7.2 \\
6.9 \\
6.1\end{array}$ & $\begin{array}{r}308 \\
148 \\
119 \\
108 \\
64 \\
109 \\
56 \\
42 \\
54 \\
39\end{array}$ & $\begin{array}{r}9.5 \\
5.5 \\
11.5 \\
8.5 \\
13.0 \\
11.5 \\
12.0 \\
11.5 \\
12.0 \\
11.0\end{array}$ & $\begin{array}{l}-- \\
-- \\
- \\
6.3 \\
-- \\
5.5 \\
-- \\
-- \\
9.8 \\
8.1\end{array}$ \\
\hline $\begin{array}{l}16065 \\
16067 \\
16070 \\
16075 \\
16080 \\
16085 \\
16090 \\
16095 \\
16100 \\
16105\end{array}$ & $\begin{array}{l}\text { Stream } \\
\text { Seep } \\
\text { Seep } \\
\text { Stream } \\
\text { Seep } \\
\text { Stream } \\
\text { Stream } \\
\text { Seep } \\
\text { Seep } \\
\text { Stream }\end{array}$ & $\begin{array}{l}3 / 19 / 94 \\
3 / 19 / 94 \\
3 / 19 / 94 \\
3 / 19 / 94 \\
3 / 19 / 94 \\
3 / 19 / 94 \\
3 / 19 / 94 \\
3 / 19 / 94 \\
3 / 19 / 94 \\
3 / 19 / 94\end{array}$ & $\begin{array}{l}\text { P } \\
\text { L } \\
\text { V } \\
D \\
\text { L } \\
\text { L } \\
\text { L } \\
\text { L } \\
\text { L } \\
\text { E }\end{array}$ & $\begin{array}{l}.04 \\
0 \\
0 \\
0 \\
0 \\
0 \\
0 \\
0 \\
0 \\
.02\end{array}$ & $\begin{array}{l}7 \\
6.7 \\
6.7 \\
-- \\
6.1 \\
7.3 \\
6.8 \\
6.3 \\
6.6 \\
7.1\end{array}$ & $\begin{array}{r}49 \\
135 \\
35 \\
-- \\
48 \\
105 \\
103 \\
110 \\
254 \\
118\end{array}$ & $\begin{array}{r}9.5 \\
10.5 \\
11.0 \\
- \\
10.0 \\
6.5 \\
7.5 \\
17.0 \\
8.5 \\
9.0\end{array}$ & $\begin{array}{l}-- \\
8.2 \\
11.3 \\
-- \\
- \\
-- \\
-- \\
- \\
9.1 \\
--\end{array}$ \\
\hline $\begin{array}{l}16110 \\
17005 \\
17010 \\
17015 \\
17020 \\
17030 \\
17035 \\
17040 \\
17045 \\
17050\end{array}$ & $\begin{array}{l}\text { Seep } \\
\text { Stream } \\
\text { Stream } \\
\text { Stream } \\
\text { Stream } \\
\text { Stream } \\
\text { Seep } \\
\text { Stream } \\
\text { Seep } \\
\text { Stream }\end{array}$ & $\begin{array}{l}3 / 19 / 94 \\
3 / 19 / 94 \\
3 / 19 / 94 \\
3 / 19 / 94 \\
3 / 19 / 94 \\
3 / 19 / 94 \\
3 / 19 / 94 \\
3 / 19 / 94 \\
3 / 19 / 94 \\
3 / 19 / 94\end{array}$ & $\begin{array}{l}\text { L } \\
P \\
\text { L } \\
\text { L } \\
\text { E } \\
\text { L } \\
\text { L } \\
\text { L } \\
D \\
\text { L }\end{array}$ & $\begin{array}{l}0 \\
.1 \\
0 \\
0 \\
.01 \\
0 \\
0 \\
0 \\
0 \\
0\end{array}$ & $\begin{array}{l}6.8 \\
7.4 \\
8 \\
7.4 \\
7.4 \\
7.4 \\
7.1 \\
6.5 \\
7.5\end{array}$ & $\begin{array}{r}199 \\
197 \\
305 \\
158 \\
141 \\
188 \\
143 \\
61 \\
-- \\
131\end{array}$ & $\begin{array}{r}8.5 \\
10.0 \\
10.5 \\
8.5 \\
9.0 \\
9.5 \\
10.0 \\
7.5 \\
-- \\
9.5\end{array}$ & $\begin{array}{l}4.6 \\
-- \\
- \\
- \\
-- \\
\overline{7.8} \\
-- \\
-- \\
--\end{array}$ \\
\hline $\begin{array}{l}17055 \\
17065 \\
17070 \\
17075 \\
17082 \\
17083 \\
17085 \\
17090 \\
17095 \\
17100\end{array}$ & $\begin{array}{l}\text { Seep } \\
\text { Seep } \\
\text { Seep } \\
\text { Stream } \\
\text { Seep } \\
\text { Seep } \\
\text { Stream } \\
\text { Seep } \\
\text { Stream } \\
\text { Seep }\end{array}$ & $\begin{array}{l}3 / 19 / 94 \\
3 / 19 / 94 \\
3 / 19 / 94 \\
3 / 19 / 94 \\
3 / 19 / 94 \\
3 / 19 / 94 \\
3 / 19 / 94 \\
3 / 19 / 94 \\
3 / 19 / 94 \\
3 / 19 / 94\end{array}$ & $\begin{array}{l}\text { D } \\
\text { D } \\
\text { P } \\
\text { D } \\
\text { D } \\
\text { D } \\
\text { E } \\
\text { E } \\
\text { P } \\
\text { D }\end{array}$ & $\begin{array}{l}0 \\
0 \\
.07 \\
0 \\
0 \\
0 \\
.01 \\
.01 \\
.05 \\
0\end{array}$ & $\begin{array}{l}-- \\
\overline{7.6} \\
-- \\
-- \\
\ddot{7.3} \\
6.9 \\
7.5 \\
--\end{array}$ & $\begin{array}{l}-- \\
-- \\
63 \\
-- \\
-- \\
\overline{92} \\
68 \\
71 \\
--\end{array}$ & $\begin{array}{l}-- \\
-- \\
11.5 \\
-- \\
-- \\
-- \\
9.5 \\
10.0 \\
12.5 \\
--\end{array}$ & $\begin{array}{l}-- \\
-- \\
-- \\
-- \\
-- \\
-- \\
- \\
4.4 \\
-- \\
--\end{array}$ \\
\hline $\begin{array}{l}17105 \\
17110 \\
17115 \\
17120 \\
17125 \\
17130 \\
17135 \\
18005 \\
18010 \\
18015\end{array}$ & $\begin{array}{l}\text { Stream } \\
\text { Stream } \\
\text { Seep } \\
\text { Seep } \\
\text { Stream } \\
\text { Seep } \\
\text { Seep } \\
\text { Stream } \\
\text { Seep } \\
\text { Seep }\end{array}$ & $\begin{array}{l}3 / 19 / 94 \\
3 / 19 / 94 \\
3 / 19 / 94 \\
3 / 19 / 94 \\
3 / 19 / 94 \\
3 / 19 / 94 \\
3 / 19 / 94 \\
3 / 16 / 94 \\
3 / 16 / 94 \\
3 / 16 / 94\end{array}$ & $\begin{array}{l}P \\
P \\
L \\
D \\
E \\
L \\
L \\
P \\
E \\
D\end{array}$ & $\begin{array}{l}.04 \\
.01 \\
0 \\
0 \\
.01 \\
0 \\
0 \\
.02 \\
.01 \\
0\end{array}$ & $\begin{array}{l}7.1 \\
6.7 \\
6.3 \\
-- \\
7.4 \\
6.7 \\
5.8 \\
7.6 \\
6.5 \\
-.\end{array}$ & $\begin{array}{r}41 \\
58 \\
47 \\
-- \\
55 \\
60 \\
26 \\
170 \\
90 \\
--\end{array}$ & $\begin{array}{r}12.5 \\
11.5 \\
15.0 \\
-- \\
12.0 \\
11.5 \\
12.0 \\
7.5 \\
9.0 \\
--\end{array}$ & 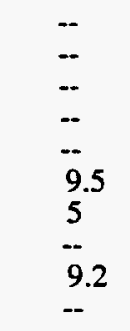 \\
\hline
\end{tabular}


Table 3. Discharge and water-quality data for the high base flow seepage investigation at Bear Creek Valley, Oak Ridge, Tennessee, March 14 through March 19, 1994-Continued

\begin{tabular}{|c|c|c|c|c|c|c|c|c|}
\hline $\begin{array}{c}\text { Site } \\
\text { number }\end{array}$ & $\begin{array}{c}\text { Type of } \\
\text { site }\end{array}$ & $\begin{array}{c}\text { Date } \\
\text { sampled } \\
\text { (month/ } \\
\text { day/year) }\end{array}$ & Method & $\begin{array}{l}\text { Flow } \\
\left(\mathrm{ft}^{3} / \mathrm{s}\right)\end{array}$ & pH & $\begin{array}{c}\text { Specific } \\
\text { conduc- } \\
\text { tance } \\
(\mu \mathrm{S} / \mathrm{cm})\end{array}$ & $\begin{array}{c}\text { Temper- } \\
\text { ature } \\
\left({ }^{\circ} \mathrm{C}\right)\end{array}$ & $\begin{array}{c}\text { Dis- } \\
\text { solved } \\
\text { oxygen } \\
\text { (mg/L) }\end{array}$ \\
\hline $\begin{array}{l}18020 \\
18025 \\
18060 \\
18065 \\
18070 \\
18075 \\
18080 \\
18085 \\
18090 \\
18095\end{array}$ & $\begin{array}{l}\text { Stream } \\
\text { Seep } \\
\text { Stream } \\
\text { Seep } \\
\text { Seep } \\
\text { Seep } \\
\text { Seep } \\
\text { Spring } \\
\text { Seep } \\
\text { Seep }\end{array}$ & $\begin{array}{l}3 / 16 / 94 \\
3 / 16 / 94 \\
3 / 16 / 94 \\
3 / 16 / 94 \\
3 / 16 / 94 \\
3 / 16 / 94 \\
3 / 16 / 94 \\
3 / 16 / 94 \\
3 / 16 / 94 \\
3 / 16 / 94\end{array}$ & $\begin{array}{l}\text { P } \\
\text { L } \\
\text { P } \\
\text { E } \\
\text { L } \\
\text { L } \\
\text { D } \\
\text { P } \\
\text { D } \\
\text { L }\end{array}$ & $\begin{array}{l}.01 \\
0 \\
.13 \\
.01 \\
0 \\
0 \\
0 \\
.09 \\
0 \\
0\end{array}$ & \begin{tabular}{c}
7.3 \\
6.1 \\
8.3 \\
7.1 \\
7.4 \\
6.4 \\
\hdashline- \\
7.6 \\
-- \\
7.2
\end{tabular} & $\begin{array}{r}90 \\
74 \\
190 \\
163 \\
268 \\
62 \\
-- \\
245 \\
-- \\
224\end{array}$ & $\begin{array}{r}8.0 \\
9.0 \\
10.0 \\
10.5 \\
11.0 \\
11.5 \\
\ddot{13.5} \\
\ddot{7} .5\end{array}$ & $\begin{array}{c}\overline{2} \\
-\overline{8.1} \\
- \\
10.1 \\
-\overline{9} \\
-\overline{5} \\
5.6\end{array}$ \\
\hline $\begin{array}{l}18100 \\
18105 \\
18110 \\
18115 \\
18120 \\
18125 \\
18130 \\
18140 \\
18145 \\
18150\end{array}$ & $\begin{array}{l}\text { Stream } \\
\text { Stream } \\
\text { Stream } \\
\text { Seep } \\
\text { Seep } \\
\text { Seep } \\
\text { Stream } \\
\text { Seep } \\
\text { Seep } \\
\text { Stream }\end{array}$ & $\begin{array}{l}3 / 16 / 94 \\
3 / 16 / 94 \\
3 / 16 / 94 \\
3 / 16 / 94 \\
3 / 16 / 94 \\
3 / 16 / 94 \\
3 / 16 / 94 \\
3 / 16 / 94 \\
3 / 16 / 94 \\
3 / 16 / 94\end{array}$ & $\begin{array}{l}P \\
P \\
P \\
D \\
D \\
D \\
L \\
D \\
L \\
P\end{array}$ & $\begin{array}{l}.12 \\
.02 \\
.02 \\
0 \\
0 \\
0 \\
0 \\
0 \\
0 \\
.1\end{array}$ & $\begin{array}{l}7.5 \\
7.4 \\
6.9 \\
-- \\
-- \\
-- \\
6.9 \\
-- \\
7 \\
7.4\end{array}$ & $\begin{array}{r}100 \\
104 \\
120 \\
- \\
-- \\
-- \\
123 \\
-- \\
50 \\
61\end{array}$ & $\begin{array}{c}6.5 \\
6.0 \\
6.5 \\
-- \\
-- \\
- \\
8.5 \\
-- \\
10.0 \\
6.5\end{array}$ & $\begin{array}{l}-- \\
-- \\
-- \\
-- \\
-- \\
-- \\
-- \\
-- \\
-- \\
--\end{array}$ \\
\hline $\begin{array}{l}18155 \\
18160 \\
18165 \\
18170 \\
18175 \\
18180 \\
18185 \\
18190 \\
18195 \\
18215\end{array}$ & $\begin{array}{l}\text { Seep } \\
\text { Stream } \\
\text { Stream } \\
\text { Stream } \\
\text { Seep } \\
\text { Seep } \\
\text { Stream } \\
\text { Seep } \\
\text { Stream } \\
\text { Seep }\end{array}$ & $\begin{array}{l}3 / 16 / 94 \\
3 / 16 / 94 \\
3 / 16 / 94 \\
3 / 16 / 94 \\
3 / 16 / 94 \\
3 / 16 / 94 \\
3 / 16 / 94 \\
3 / 16 / 94 \\
3 / 16 / 94 \\
3 / 16 / 94\end{array}$ & $\begin{array}{l}\text { L } \\
P \\
P \\
E \\
D \\
E \\
P \\
L \\
E \\
E\end{array}$ & $\begin{array}{l}0 \\
.11 \\
.02 \\
.01 \\
0^{0} .01 \\
.03 \\
.03 \\
0^{.01}\end{array}$ & $\begin{array}{l}6.7 \\
7.5 \\
6.5 \\
6.5 \\
-- \\
6 \\
7.7 \\
\overline{7.8} \\
6.8\end{array}$ & $\begin{array}{r}118 \\
50 \\
31 \\
36 \\
-- \\
33 \\
86 \\
-- \\
90 \\
133\end{array}$ & $\begin{array}{c}5.0 \\
5.5 \\
8.0 \\
9.5 \\
- \\
10.5 \\
8.5 \\
- \\
9.5 \\
10.0\end{array}$ & $\begin{array}{l}3.4 \\
-- \\
-- \\
-- \\
\overline{8.3} \\
-- \\
-- \\
-\overline{3}\end{array}$ \\
\hline $\begin{array}{l}18220 \\
18225 \\
18230 \\
18250 \\
18255 \\
18260 \\
19003 \\
19004 \\
19005 \\
19007\end{array}$ & $\begin{array}{l}\text { Seep } \\
\text { Seep } \\
\text { Seep } \\
\text { Stream } \\
\text { Seep } \\
\text { Stream } \\
\text { Spring } \\
\text { Stream } \\
\text { Seep } \\
\text { Spring }\end{array}$ & $\begin{array}{l}3 / 16 / 94 \\
3 / 16 / 94 \\
3 / 16 / 94 \\
3 / 16 / 94 \\
3 / 16 / 94 \\
3 / 16 / 94 \\
3 / 17 / 94 \\
3 / 18 / 94 \\
3 / 18 / 94 \\
3 / 18 / 94\end{array}$ & $\begin{array}{l}\text { L } \\
\text { L } \\
\text { E } \\
\text { P } \\
\text { D } \\
\text { P } \\
\text { E } \\
\text { D } \\
\text { D } \\
\text { E }\end{array}$ & $\begin{array}{l}0 \\
0 \\
.01 \\
.04 \\
0 \\
.13 \\
.02 \\
0 \\
0 \\
.03\end{array}$ & $\begin{array}{l}6.7 \\
5.9 \\
6 \\
8 \\
- \\
7.5 \\
7.6 \\
-- \\
-- \\
7.4\end{array}$ & $\begin{array}{r}83 \\
43 \\
36 \\
191 \\
- \\
188 \\
316 \\
-- \\
- \\
242\end{array}$ & $\begin{array}{c}11 \\
8.5 \\
10.0 \\
7.0 \\
-- \\
9.5 \\
13.5 \\
-- \\
-- \\
11.5\end{array}$ & $\begin{array}{c}9.5 \\
5.8 \\
5.8 \\
-- \\
-- \\
- \\
10.2 \\
-- \\
\overline{8.2}\end{array}$ \\
\hline $\begin{array}{l}19008 \\
19010 \\
19015 \\
19045 \\
19050 \\
19055 \\
19090 \\
19095 \\
19100 \\
19105\end{array}$ & $\begin{array}{l}\text { Stream } \\
\text { Seep } \\
\text { Stream } \\
\text { Stream } \\
\text { Stream } \\
\text { Seep } \\
\text { Seep } \\
\text { Stream } \\
\text { Stream } \\
\text { Stream }\end{array}$ & $\begin{array}{l}3 / 18 / 94 \\
3 / 17 / 94 \\
3 / 18 / 94 \\
3 / 18 / 94 \\
3 / 18 / 94 \\
3 / 18 / 94 \\
3 / 18 / 94 \\
3 / 18 / 94 \\
3 / 18 / 94 \\
3 / 18 / 94\end{array}$ & $\begin{array}{l}\text { P } \\
\text { D } \\
\text { P } \\
\text { P } \\
\text { P } \\
\text { L } \\
\text { D } \\
\text { L } \\
\text { P } \\
\text { E }\end{array}$ & $\begin{array}{l}0.14 \\
0 \\
.16 \\
.01 \\
-- \\
0 \\
0 \\
0 \\
.1 \\
.-\end{array}$ & $\begin{array}{l}8 \\
-8 \\
7.3 \\
7.5 \\
7.4 \\
-\overline{8} \\
7.8 \\
7.6\end{array}$ & $\begin{array}{r}169 \\
-- \\
171 \\
186 \\
400 \\
186 \\
-- \\
328 \\
125 \\
711\end{array}$ & $\begin{array}{c}13.0 \\
-\overline{13.5} \\
10.5 \\
9.5 \\
8.0 \\
- \\
10.0 \\
12.0 \\
12.5\end{array}$ & $\begin{array}{l}-- \\
-- \\
-- \\
-- \\
-- \\
8.5 \\
-- \\
-- \\
-- \\
--\end{array}$ \\
\hline
\end{tabular}


Table 3. Discharge and water-quality data for the high base flow seepage investigation at Bear Creek Valley, Oak Ridge, Tennessee, March 14 through March 19, 1994-Continued

\begin{tabular}{|c|c|c|c|c|c|c|c|c|}
\hline $\begin{array}{c}\text { Site } \\
\text { number }\end{array}$ & $\begin{array}{l}\text { Type of } \\
\text { site }\end{array}$ & $\begin{array}{c}\text { Date } \\
\text { sampled } \\
\text { (month/ } \\
\text { day/year) }\end{array}$ & Method & $\begin{array}{l}\text { Flow } \\
\left(\mathrm{ft}^{3} / \mathrm{s}\right)\end{array}$ & $\mathbf{p H}$ & $\begin{array}{c}\text { Specific } \\
\text { conduc- } \\
\text { tance } \\
(\mu \mathrm{S} / \mathrm{cm})\end{array}$ & $\begin{array}{c}\text { Temper- } \\
\text { ature } \\
\left({ }^{\circ} \mathrm{C}\right)\end{array}$ & $\begin{array}{c}\text { Dis- } \\
\text { solved } \\
\text { oxygen } \\
\text { (mg/L) }\end{array}$ \\
\hline 19110 & Seep & $3 / 18 / 94$ & D & 0 & -. & - & - & - \\
\hline 19115 & Stream & $3 / 18 / 94$ & V & .18 & 7.9 & 118 & 8.5 & - \\
\hline 19120 & Seep & $3 / 18 / 94$ & D & 0 & -- & - & -- & -- \\
\hline 19125 & Wetland & $3 / 18 / 94$ & $\mathrm{D}$ & 0 & .. & - & -. & - \\
\hline 19130 & Seep & $3 / 18 / 94$ & D & 0 & - & - & -- & - \\
\hline 19135 & Stream & $3 / 18 / 94$ & $\mathbf{P}$ & .12 & 7.6 & 81 & 7.0 & - \\
\hline 19140 & Stream & $3 / 18 / 94$ & $\mathbf{P}$ & .05 & 7.3 & 42 & 7.5 & - \\
\hline 19145 & Stream & $3 / 18 / 94$ & $L$ & 0 & 6.8 & 136 & 7.0 & -- \\
\hline 19146 & Stream & $3 / 18 / 94$ & $\bar{D}$ & 0 & - & - & -- & - \\
\hline 19148 & Seep & $3 / 18 / 94$ & $\mathbf{L}$ & 0 & 5.8 & 40 & 9.5 & -- \\
\hline 19150 & Seep & $3 / 18 / 94$ & $\mathrm{D}$ & 0 & 7.1 & 199 & 7.5 & 9.6 \\
\hline 19155 & Stream & $3 / 18 / 94$ & $\bar{E}$ & .01 & 5.6 & 42 & 8.5 & $\therefore$ \\
\hline 19156 & Stream & $3 / 18 / 94$ & $\mathrm{~L}$ & 0 & 5.6 & 38 & 9.0 & - \\
\hline 19158 & Seep & $3 / 18 / 94$ & $\bar{D}$ & 0 & - & - & -- & - \\
\hline 19160 & Stream & $3 / 18 / 94$ & D & 0 & - & - & -- & - \\
\hline 19165 & Seep & $3 / 18 / 94$ & D & 0 & -- & $\cdots$ & - & - \\
\hline 19170 & Seep & $3 / 18 / 94$ & D & 0 & -- & - & 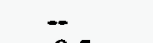 & -- \\
\hline 19175 & Seep & $3 / 18 / 94$ & $\bar{L}$ & 0 & 6 & 37 & 8.5 & - \\
\hline 19180 & Stream & $3 / 18 / 94$ & $\mathbf{P}$ & .05 & 7.9 & 166 & 10.0 & - \\
\hline 19285 & Stream & $3 / 18 / 94$ & $P$ & .14 & 8.4 & 119 & 12.5 & -- \\
\hline 19290 & Stream & $3 / 18 / 94$ & D & 0 & -. & -- & - & - \\
\hline 19295 & Seep & $3 / 18 / 94$ & $\overline{\mathbf{L}}$ & 0 & 7.3 & 150 & 11.5 & -. \\
\hline 19300 & Stream & $3 / 18 / 94$ & $\overrightarrow{\mathbf{P}}$ & .23 & 8.1 & 167 & 13.0 & - \\
\hline 20005 & Stream & $3 / 18 / 94$ & $\mathbf{P}$ & 2.11 & 8.3 & 219 & 10.0 & - \\
\hline 20010 & Stream & $3 / 18 / 94$ & $\mathbf{P}$ & .18 & 7.7 & 128 & 11.0 & -- \\
\hline 20015 & Stream & $3 / 18 / 94$ & $\mathbf{P}$ & -- & 7.1 & 72 & 11.0 & -- \\
\hline 20020 & Seep & $3 / 18 / 94$ & $\mathbf{L}$ & 0 & 5.7 & 42 & 10.5 & 5.7 \\
\hline 20250 & Stream & $3 / 19 / 94$ & $\overline{\mathbf{P}}$ & 1.73 & 8.3 & 222 & 10.0 & - \\
\hline 20255 & Stream & $3 / 19 / 94$ & $\mathbf{P}$ & 1.42 & 8.2 & 293 & 11.5 & - \\
\hline 20258 & Seep & $3 / 19 / 94$ & L & 0 & 7.6 & 395 & 10.5 & 3.1 \\
\hline 20260 & Stream & $3 / 19 / 94$ & $\mathbf{P}$ & 1.34 & 8 & 223 & 12.5 & -- \\
\hline 20265 & Stream & $3 / 19 / 94$ & $\mathbf{P}$ & .11 & 7.6 & 112 & 13.0 & - \\
\hline 20280 & Stream & $3 / 18 / 94$ & $\mathbf{P}$ & .06 & 7.3 & 80 & 12.5 & -- \\
\hline 20285 & Seep & $3 / 18 / 94$ & $\mathbf{E}$ & $-\infty$ & 6.9 & 137 & 12.0 & 4.8 \\
\hline 20290 & Stream & $3 / 18 / 94$ & $\mathbf{P}$ & .06 & 7 & 59 & 10.5 & - \\
\hline 20295 & Seep & $3 / 18 / 94$ & $\dot{L}$ & 0 & 5.9 & 54 & 8.5 & - \\
\hline 20300 & Seep & $3 / 18 / 94$ & $\mathbf{E}$ & .01 & 6.2 & 37 & 8.5 & 9.2 \\
\hline 20305 & Stream & $3 / 19 / 94$ & $\mathbf{P}$ & .02 & 7.2 & 58 & 7.0 & $\cdots$ \\
\hline 20310 & Seep & $3 / 19 / 94$ & $\mathbf{E}$ & -- & 6.4 & 60 & 7.5 & 3.4 \\
\hline 20315 & Seep & $3 / 18 / 94$ & $\mathbf{L}$ & 0 & 6.5 & 69 & 10.0 & 8.9 \\
\hline 20320 & Seep & $3 / 19 / 94$ & $\mathbf{L}$ & 0 & 5.7 & 43 & 7.5 & 2 \\
\hline 20325 & Stream & $3 / 19 / 94$ & $\mathbf{P}$ & .01 & 7 & 69 & 7.0 & - \\
\hline 20335 & Seep & $3 / 19 / 94$ & $\mathbf{E}$ & .01 & 7.2 & 74 & 9.5 & 9.6 \\
\hline 20340 & Stream & $3 / 18 / 94$ & $\mathbf{E}$ & .01 & 7.3 & 104 & 9.0 & - \\
\hline 20342 & Stream & $3 / 18 / 94$ & $\mathbf{P}$ & .01 & 7.2 & 114 & 11.0 & - \\
\hline 20343 & Stream & $3 / 18 / 94$ & L & 0 & 7.3 & 100 & 15.5 & - \\
\hline 20350 & Stream & $3 / 18 / 94$ & $\mathbf{P}$ & .09 & 7.3 & 92 & 12.0 & - \\
\hline 20355 & Seep & $3 / 18 / 94$ & $\mathrm{E}$ & .01 & 6.9 & 184 & 11.0 & 7 \\
\hline 20360 & Stream & $3 / 18 / 94$ & $\mathbf{P}$ & .02 & 6.8 & 42 & 11.5 & - \\
\hline 20365 & Stream & $3 / 18 / 94$ & E & .01 & 6 & 32 & 10.5 & -- \\
\hline
\end{tabular}


Table 3. Discharge and water-quality data for the high base flow seepage investigation at Bear Creek Valley, Oak Ridge, Tennessee, March 14 through March 19, 1994-Continued

\begin{tabular}{|c|c|c|c|c|c|c|c|c|}
\hline $\begin{array}{c}\text { Site } \\
\text { number }\end{array}$ & $\begin{array}{l}\text { Type of } \\
\text { site }\end{array}$ & $\begin{array}{c}\text { Date } \\
\text { sampled } \\
\text { (month/ } \\
\text { day/year) }\end{array}$ & Method & $\begin{array}{l}\text { Flow } \\
\left(t^{3} / s\right)\end{array}$ & pH & $\begin{array}{c}\text { Specific } \\
\text { conduc- } \\
\text { tance } \\
(\mu \mathrm{S} / \mathrm{cm})\end{array}$ & $\begin{array}{c}\text { Temper- } \\
\text { ature } \\
\left({ }^{\circ} \mathrm{C}\right)\end{array}$ & $\begin{array}{c}\text { Dis- } \\
\text { solved } \\
\text { oxygen } \\
\text { (mg/L) }\end{array}$ \\
\hline $\begin{array}{l}20370 \\
20375 \\
20380 \\
20385 \\
20390 \\
20395 \\
20400 \\
20405 \\
20410 \\
20415\end{array}$ & $\begin{array}{l}\text { Stream } \\
\text { Seep } \\
\text { Seep } \\
\text { Seep } \\
\text { Stream } \\
\text { Seep } \\
\text { Seep } \\
\text { Seep } \\
\text { Stream } \\
\text { Seep }\end{array}$ & $\begin{array}{l}3 / 18 / 94 \\
3 / 18 / 94 \\
3 / 18 / 94 \\
3 / 18 / 94 \\
3 / 18 / 94 \\
3 / 18 / 94 \\
3 / 18 / 94 \\
3 / 18 / 94 \\
3 / 18 / 94 \\
3 / 18 / 94\end{array}$ & $\begin{array}{l}E \\
D \\
E \\
L \\
E \\
D \\
L \\
D \\
P \\
L\end{array}$ & $\begin{array}{l}.02 \\
0.05 \\
0 \\
.01 \\
0 \\
0 \\
0 \\
.02 \\
0\end{array}$ & $\begin{array}{l}5.8 \\
-5.3 \\
6.7 \\
6.9 \\
- \\
6.8 \\
-7 \\
7.2\end{array}$ & $\begin{array}{l}30 \\
-- \\
28 \\
62 \\
32 \\
-- \\
44 \\
-- \\
49 \\
51\end{array}$ & $\begin{array}{c}9.0 \\
\ddot{9.5} \\
9.0 \\
8.0 \\
\ddot{10.5} \\
-\overline{8.5} \\
11.5\end{array}$ & $\begin{array}{l}-- \\
-\overline{5.7} \\
6.3 \\
- \\
- \\
9.5 \\
-\overline{10.5} \\
10\end{array}$ \\
\hline $\begin{array}{l}20420 \\
20425 \\
20430 \\
21005 \\
21010 \\
21015 \\
21020 \\
21025 \\
21030 \\
21035\end{array}$ & $\begin{array}{l}\text { Stream } \\
\text { Stream } \\
\text { Stream } \\
\text { Stream } \\
\text { Seep } \\
\text { Stream } \\
\text { Spring } \\
\text { Stream } \\
\text { Spring } \\
\text { Stream }\end{array}$ & $\begin{array}{l}3 / 18 / 94 \\
3 / 18 / 94 \\
3 / 18 / 94 \\
3 / 19 / 94 \\
3 / 19 / 94 \\
3 / 19 / 94 \\
3 / 19 / 94 \\
3 / 19 / 94 \\
3 / 19 / 94 \\
3 / 19 / 94\end{array}$ & $\begin{array}{l}E \\
L \\
P \\
P \\
L \\
P \\
L \\
L \\
L \\
P\end{array}$ & $\begin{array}{l}.01 \\
0.21 \\
1.21 \\
.04 \\
0 \\
.04 \\
0 \\
0 \\
0 \\
.04\end{array}$ & $\begin{array}{l}5.6 \\
5.9 \\
7.5 \\
6.5 \\
5.2 \\
7.1 \\
7.1 \\
6.4 \\
5.8 \\
7\end{array}$ & $\begin{array}{r}30 \\
30 \\
231 \\
28 \\
29 \\
88 \\
65 \\
64 \\
47 \\
51\end{array}$ & $\begin{array}{r}8.5 \\
10.5 \\
12.0 \\
7.5 \\
9.0 \\
7.5 \\
7.5 \\
8.0 \\
9.0 \\
7.5\end{array}$ & $\begin{array}{c}-- \\
6.7 \\
-- \\
-- \\
6.7 \\
-\overline{10.1} \\
-- \\
4 \\
--\end{array}$ \\
\hline $\begin{array}{l}21040 \\
21045 \\
21050 \\
21055 \\
21057 \\
21058 \\
21060 \\
21065 \\
21100 \\
21102\end{array}$ & $\begin{array}{l}\text { Seep } \\
\text { Stream } \\
\text { Seep } \\
\text { Stream } \\
\text { Stream } \\
\text { Seep } \\
\text { Spring } \\
\text { Stream } \\
\text { Stream } \\
\text { Stream }\end{array}$ & $\begin{array}{l}3 / 19 / 94 \\
3 / 19 / 94 \\
3 / 19 / 94 \\
3 / 19 / 94 \\
3 / 19 / 94 \\
3 / 19 / 94 \\
3 / 19 / 94 \\
3 / 19 / 94 \\
3 / 19 / 94 \\
3 / 19 / 94\end{array}$ & $\begin{array}{l}\text { L } \\
\text { L } \\
\text { E } \\
\text { E } \\
\text { L } \\
\text { D } \\
\text { E } \\
\text { L } \\
\text { P } \\
\text { L }\end{array}$ & $\begin{array}{l}0 \\
0 \\
.01 \\
.01 \\
0 \\
0 \\
.01 \\
0 \\
.05 \\
0\end{array}$ & $\begin{array}{l}6.2 \\
6.1 \\
6.9 \\
7.3 \\
6.3 \\
6 \\
5.5 \\
7.5 \\
7.1\end{array}$ & $\begin{array}{r}68 \\
37 \\
30 \\
42 \\
41 \\
- \\
22 \\
32 \\
107 \\
97\end{array}$ & $\begin{array}{r}7.0 \\
8.5 \\
11.0 \\
13.0 \\
11.0 \\
-12.5 \\
9.5 \\
13.5 \\
9.5\end{array}$ & $\begin{array}{l}3.4 \\
-- \\
-- \\
- \\
- \\
9 \\
- \\
-\end{array}$ \\
\hline $\begin{array}{l}21103 \\
21105 \\
21110 \\
21115 \\
21119 \\
21120 \\
21125 \\
21130 \\
22005 \\
22006\end{array}$ & $\begin{array}{l}\text { Seep } \\
\text { Stream } \\
\text { Seep } \\
\text { Seep } \\
\text { Seep } \\
\text { Stream } \\
\text { Stream } \\
\text { Seep } \\
\text { Stream } \\
\text { Seep }\end{array}$ & $\begin{array}{r}3 / 19 / 94 \\
3 / 19 / 94 \\
3 / 19 / 94 \\
3 / 19 / 94 \\
-7 \\
3 / 19 / 94 \\
3 / 19 / 94 \\
3 / 19 / 94 \\
3 / 18 / 94 \\
3 / 18 / 94\end{array}$ & $\begin{array}{l}\text { L } \\
P \\
\text { L } \\
D \\
P \\
P \\
\text { L } \\
\text { D } \\
\text { P } \\
\text { L }\end{array}$ & $\begin{array}{l}0 \\
.02 \\
0 \\
0 \\
-.01 \\
.0 \\
0 . \\
0.22 \\
0 .\end{array}$ & $\begin{array}{l}6.5 \\
7.9 \\
-- \\
-- \\
\overline{7} \\
7.6 \\
7.3 \\
-\overline{7.4} \\
7.1\end{array}$ & $\begin{array}{r}85 \\
104 \\
-. \\
\ddot{-} \\
129 \\
125 \\
\ddot{84} \\
151\end{array}$ & $\begin{array}{c}13.5 \\
13.5 \\
-- \\
- \\
- \\
15.0 \\
11.5 \\
-\ddot{14.4} \\
20.6\end{array}$ & $\begin{array}{l}5.5 \\
-- \\
-- \\
-- \\
-- \\
-- \\
-\overline{9.3} \\
--\end{array}$ \\
\hline $\begin{array}{l}22010 \\
22034 \\
22040 \\
22041 \\
22042 \\
22044 \\
22045 \\
22050 \\
22055 \\
22060\end{array}$ & $\begin{array}{l}\text { Stream } \\
\text { Seep } \\
\text { Stream } \\
\text { Seep } \\
\text { Stream } \\
\text { Stream } \\
\text { Stream } \\
\text { Stream } \\
\text { Stream } \\
\text { Stream }\end{array}$ & $\begin{array}{l}3 / 19 / 94 \\
3 / 19 / 94 \\
3 / 19 / 94 \\
3 / 19 / 94 \\
3 / 19 / 94 \\
3 / 19 / 94 \\
3 / 19 / 94 \\
3 / 19 / 94 \\
3 / 19 / 94 \\
3 / 19 / 94\end{array}$ & $\begin{array}{l}\mathbf{E} \\
\mathbf{L} \\
\mathrm{E} \\
\mathrm{D} \\
\mathrm{D} \\
\mathrm{E} \\
\mathbf{P} \\
\mathrm{P} \\
\mathrm{E} \\
\mathbf{P}\end{array}$ & $\begin{array}{l}.3 \\
0 \\
.02 \\
0 \\
0 \\
.01 \\
1.54 \\
.07 \\
.01 \\
.07\end{array}$ & $\begin{array}{l}8.2 \\
7.4 \\
7.4 \\
-- \\
-7.1 \\
8.2 \\
7.6 \\
7.1 \\
7.8 \\
\end{array}$ & $\begin{array}{r}192 \\
770 \\
223 \\
-- \\
-- \\
98 \\
221 \\
247 \\
440 \\
182\end{array}$ & $\begin{array}{c}11.5 \\
10.0 \\
11.0 \\
- \\
- \\
9.0 \\
12.4 \\
13.5 \\
11.0 \\
12.5 \\
\end{array}$ & $\begin{array}{l}- \\
- \\
-- \\
-- \\
-- \\
-- \\
- \\
-\end{array}$ \\
\hline
\end{tabular}


Table 5. Discharge and water-quality data for the low base flow seepage investigation at Bear Creek Valley, Oak Ridge, Tennessee, September 9 through September 29, 1994

[Methods for measurement: D, no flowing water; E, estimation; F, float: L, less than mimimum reportable flow, 0.005 cubic foot per second; $P$, pygmy meter measurement: $R$, rating from stream gage: $V$, volumetric measurement; $\mathrm{ft}^{3} / \mathrm{s}$, cubic foot per second; $\mu \mathrm{S} / \mathrm{cm}$, microsiemens per centimeter; ${ }^{\circ} \mathrm{C}$, degree Celsius; mg/L, milligrams per liter; --, no data]

\begin{tabular}{|c|c|c|c|c|c|c|c|c|}
\hline $\begin{array}{c}\text { Site } \\
\text { number }\end{array}$ & $\begin{array}{l}\text { Type of } \\
\text { site }\end{array}$ & $\begin{array}{c}\text { Date } \\
\text { sampled } \\
\text { (month/ } \\
\text { day/year) }\end{array}$ & Method & $\begin{array}{l}\text { Flow } \\
\left(\mathrm{ft}^{3} / \mathrm{s}\right)\end{array}$ & pH & $\begin{array}{l}\text { Specific } \\
\text { conduc- } \\
\text { tance } \\
(\mu \mathrm{S} / \mathrm{cm})\end{array}$ & $\begin{array}{c}\text { Temper- } \\
\text { ature } \\
\left({ }^{\circ} \mathrm{C}\right)\end{array}$ & $\begin{array}{l}\text { Dis- } \\
\text { solved } \\
\text { oxygen } \\
\text { (mg/L) }\end{array}$ \\
\hline 1005 & Stream & $9 / 29 / 94$ & $\mathrm{P}$ & 0.02 & 7.7 & 1,605 & 22.5 & -- \\
\hline 1010 & Stream & $9 / 29 / 94$ & $\mathrm{~L}$ & 0 & 7.9 & 1,936 & 21.0 & - \\
\hline 1015 & Stream & $9 / 9 / 94$ & L & 0 & 7.8 & 2,030 & 19.5 & -- \\
\hline 1020 & Stream & $9 / 9 / 94$ & $\vec{D}$ & 0 & -- & - & -- & -- \\
\hline 1025 & Stream & $9 / 9 / 94$ & D & 0 & -- & -- & -- & -- \\
\hline 1030 & Stream & $9 / 9 / 94$ & D & 0 & -- & -- & -- & -- \\
\hline 1035 & Stream & $9 / 9 / 94$ & D & 0 & -- & -- & -- & -- \\
\hline 1040 & Seep & $9 / 9 / 94$ & D & 0 & -- & $\cdots$ & -- & - \\
\hline $\begin{array}{l}1045 \\
1050\end{array}$ & $\begin{array}{l}\text { Seep } \\
\text { Stream }\end{array}$ & $\begin{array}{l}9 / 9 / 94 \\
9 / 9 / 94\end{array}$ & $\begin{array}{l}\mathbf{D} \\
\mathbf{P}\end{array}$ & $\begin{array}{l}0 \\
0\end{array}$ & $-\overline{6.9}$ & $75 \overline{5}$ & $\overline{18.0}$ & $\overline{6} .5$ \\
\hline 1055 & Seep & $9 / 9 / 94$ & D & 0 & -- & -- & - & - \\
\hline 1060 & Stream & 9/9/94 & D & 0 & -- & -- & -- & - \\
\hline 1065 & Seep & $9 / 9 / 94$ & D & 0 & -- & - & -- & -- \\
\hline 1070 & Seep & 9/9/94 & D & 0 & $\bar{z}$ & $=$ & 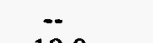 & 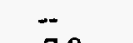 \\
\hline 1075 & Stream & $9 / 9 / 94$ & $\mathbf{L}$ & 0 & 7.9 & 259 & 18.0 & 7.8 \\
\hline 1080 & Seep & $9 / 9 / 94$ & $\overline{\mathrm{D}}$ & 0 & -- & - & -- & - \\
\hline 1085 & Stream & 9/9/94 & D & 0 & -- & -- & -- & -- \\
\hline 1090 & Stream & $9 / 9 / 94$ & D & 0 & - & -- & -- & - \\
\hline 1095 & Seep & 9/9/94 & D & 0 & -- & $\cdots$ & - & - \\
\hline 1100 & Seep & $9 / 9 / 94$ & D & 0 & -- & -- & -- & - \\
\hline 1105 & Stream & 9/9/94 & L & 0 & 7.4 & 115 & 18.5 & 7.1 \\
\hline 1110 & Stream & $9 / 9 / 94$ & D & 0 & -- & -- & - & -- \\
\hline 1115 & Seep & $9 / 9 / 94$ & D & 0 & -- & -. & -- & - \\
\hline 1120 & Stream & $9 / 9 / 94$ & D & 0 & -- & -. & -- & -- \\
\hline 1125 & Seep & $9 / 9 / 94$ & D & 0 & -- & - & - & - \\
\hline 1130 & Stream & $9 / 9 / 94$ & $\bar{L}$ & 0 & -- & 88 & 18.5 & 8.2 \\
\hline 1135 & Seep & $9 / 9 / 94$ & D & 0 & -- & -- & -- & -- \\
\hline 1140 & Stream & $9 / 9 / 94$ & $\mathbf{L}$ & 0 & 7.3 & 77 & 19.0 & 8.1 \\
\hline 1145 & Stream & $9 / 9 / 94$ & D & 0 & -- & -- & - & -- \\
\hline 1148 & Stream & $9 / 9 / 94$ & D & 0 & -- & - & -- & -- \\
\hline 1150 & Spring & $9 / 9 / 94$ & D & 0 & -- & - & - & - \\
\hline 1155 & Seep & 9/9/94 & D & 0 & -- & - & -- & -- \\
\hline 1480 & Seep & 9/9/94 & D & 0 & - & - & $\ddot{0}$ & -- \\
\hline 1485 & Stream & $9 / 9 / 94$ & $\mathbf{P}$ & .04 & 7.8 & 1,480 & 19.5 & - \\
\hline 1490 & Seep & $9 / 9 / 94$ & $\mathrm{D}$ & 0 & - & - & -- & -- \\
\hline 1495 & Stream & $9 / 9 / 94$ & $\mathbf{P}$ & .02 & 7.8 & 1,584 & 20.0 & $\cdots$ \\
\hline 1500 & Stream & 9/9/94 & $\mathbf{P}$ & .01 & 7.5 & 1,685 & 19.5 & -- \\
\hline 1502 & Spring & $9 / 9 / 94$ & L & 0 & 6.9 & 1,220 & 18.5 & 4.1 \\
\hline 1505 & Stream & $9 / 9 / 94$ & $\overline{\mathrm{L}}$ & 0 & 7.7 & 1,285 & 19.0 & $-\cdot$ \\
\hline 1510 & Stream & $9 / 9 / 94$ & $\vec{L}$ & 0 & 6.5 & 666 & 19.0 & -- \\
\hline 1515 & Stream & 9/9/94 & $E$ & .01 & 7.7 & 759 & 19.5 & - \\
\hline 1520 & Seep & $9 / 9 / 94$ & $\bar{D}$ & 0 & -- & - & - & -- \\
\hline 1525 & Spring & 9/9/94 & D & 0 & -- & -- & - & -- \\
\hline 1530 & Seep & $9 / 9 / 94$ & D & 0 & -- & - & - & -- \\
\hline 1535 & Seep & $9 / 9 / 94$ & L & 0 & 7.5 & 395 & 20.0 & -- \\
\hline 1540 & Stream & $9 / 9 / 94$ & $\mathbf{L}$ & 0 & 7.3 & 124 & 20.0 & -- \\
\hline 1545 & Seep & $9 / 9 / 94$ & D & 0 & -- & -- & -- & -- \\
\hline 1550 & Stream & 9/9/94 & $\mathbf{E}$ & .01 & 7.3 & 311 & 20.0 & $\cdots$ \\
\hline 1555 & Stream & $9 / 9 / 94$ & D & 0 & - & -- & $\overline{105}$ & 32 \\
\hline 1560 & Seep & 9/9/94 & $\mathbf{L}$ & 0 & 6.6 & 110 & 19.5 & 2.2 \\
\hline
\end{tabular}


Table 5. Discharge and water-quality data for the low base flow seepage investigation at Bear Creek Valley, Oak Ridge, Tennessee, September 9 through September 29, 1994-Continued

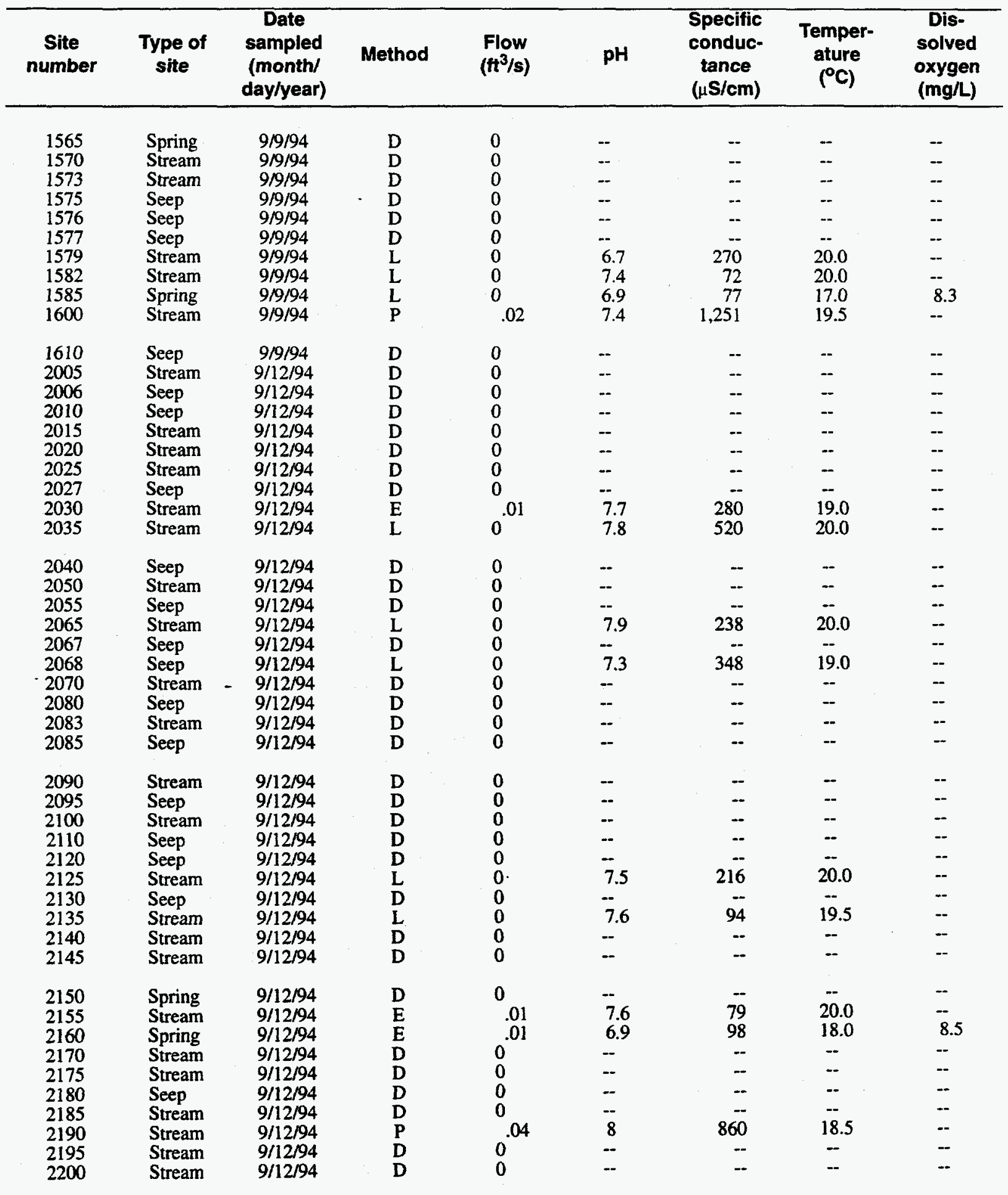


Table 5. Discharge and water-quality data for the low base flow seepage investigation at Bear Creek Valley, Oak Ridge, Tennessee, September 9 through September 29, 1994-Continued

\begin{tabular}{|c|c|c|c|c|c|c|c|c|}
\hline $\begin{array}{c}\text { Site } \\
\text { number }\end{array}$ & $\begin{array}{l}\text { Type of } \\
\text { site }\end{array}$ & $\begin{array}{c}\text { Date } \\
\text { sampled } \\
\text { (month/ } \\
\text { day/year) }\end{array}$ & Method & $\begin{array}{l}\text { Flow } \\
\left(\mathrm{ft}^{3} / \mathrm{s}\right)\end{array}$ & $\mathbf{p H}$ & $\begin{array}{c}\text { Specific } \\
\text { conduc- } \\
\text { tance } \\
(\mu \mathrm{S} / \mathrm{cm})\end{array}$ & $\begin{array}{c}\text { Temper- } \\
\text { ature } \\
\left({ }^{\circ} \mathrm{C}\right)\end{array}$ & $\begin{array}{c}\text { Dis- } \\
\text { solved } \\
\text { oxygen } \\
(\mathrm{mg} / \mathrm{L})\end{array}$ \\
\hline $\begin{array}{l}2205 \\
2210 \\
2225 \\
2230 \\
2235 \\
2240 \\
2245 \\
2260 \\
2270 \\
2275\end{array}$ & $\begin{array}{l}\text { Wetland } \\
\text { Stream } \\
\text { Stream } \\
\text { Stream } \\
\text { Stream } \\
\text { Stream } \\
\text { Stream } \\
\text { Spring } \\
\text { Seep } \\
\text { Stream }\end{array}$ & $\begin{array}{c}9 / 12 / 94 \\
9 / 12 / 94 \\
9 / 12 / 94 \\
9 / 12 / 94 \\
9 / 12 / 94 \\
9 / 12 / 94 \\
9 / 12 / 94 \\
9 / 12 / 94 \\
9 / 12 / 94 \\
9 / 9 / 94\end{array}$ & $\begin{array}{l}\text { D } \\
\text { L } \\
\text { D } \\
\text { D } \\
D \\
D \\
\text { L } \\
\text { L } \\
\text { D } \\
\text { D }\end{array}$ & $\begin{array}{l}0 \\
0 \\
0 \\
0 \\
0 \\
0 \\
0 \\
0 \\
0 \\
0\end{array}$ & $\begin{array}{l}-- \\
7.4 \\
-- \\
-- \\
-- \\
-. \\
7.5 \\
7.1 \\
-- \\
-.\end{array}$ & $\begin{array}{r}-- \\
642 \\
- \\
- \\
- \\
- \\
73 \\
84 \\
-- \\
--\end{array}$ & $\begin{array}{l}-- \\
20.0 \\
-- \\
-- \\
-- \\
-\overline{19.0} \\
18.5 \\
-- \\
--\end{array}$ & $\begin{array}{l}-- \\
-- \\
-- \\
-- \\
-- \\
-- \\
-- \\
7.4 \\
-- \\
--\end{array}$ \\
\hline $\begin{array}{l}2280 \\
2285 \\
2290 \\
2295 \\
2310 \\
2315 \\
2320 \\
2325 \\
2330 \\
2335\end{array}$ & $\begin{array}{l}\text { Seep } \\
\text { Stream } \\
\text { Stream } \\
\text { Stream } \\
\text { Seep } \\
\text { Stream } \\
\text { Stream } \\
\text { Spring } \\
\text { Spring } \\
\text { Stream }\end{array}$ & $\begin{array}{c}9 / 9 / 94 \\
9 / 9 / 94 \\
9 / 9 / 94 \\
9 / 9 / 94 \\
9 / 9 / 94 \\
9 / 12 / 94 \\
9 / 12 / 94 \\
9 / 12 / 94 \\
9 / 12 / 94 \\
9 / 12 / 94\end{array}$ & $\begin{array}{l}D \\
D \\
L \\
L \\
L \\
L \\
V \\
L \\
L \\
E\end{array}$ & $\begin{array}{l}0 \\
0 \\
0 \\
0 \\
0 \\
0 \\
.01 \\
0 \\
0 \\
.02\end{array}$ & $\begin{array}{l}-- \\
7.4 \\
7.6 \\
7.6 \\
6.9 \\
8.2 \\
8.2 \\
6.9 \\
7.5 \\
8.2\end{array}$ & $\begin{array}{r}-- \\
98 \\
90 \\
94 \\
92 \\
857 \\
354 \\
378 \\
344 \\
352\end{array}$ & $\begin{array}{l}-\overline{20.5} \\
20.0 \\
20.0 \\
17.5 \\
18.0 \\
20.5 \\
14.0 \\
15.5 \\
16.5\end{array}$ & $\begin{array}{l}-- \\
-- \\
-- \\
-- \\
8.2 \\
-- \\
-\overline{6.4} \\
8.6 \\
--\end{array}$ \\
\hline $\begin{array}{l}2337 \\
2339 \\
2341 \\
2345 \\
3005 \\
3010 \\
3015 \\
3020 \\
3025 \\
3030\end{array}$ & $\begin{array}{l}\text { Stream } \\
\text { Spring } \\
\text { Spring } \\
\text { Stream } \\
\text { Stream } \\
\text { Stream } \\
\text { Stream } \\
\text { Wetland } \\
\text { Spring } \\
\text { Spring }\end{array}$ & $\begin{array}{l}9 / 12 / 94 \\
9 / 12 / 94 \\
9 / 12 / 94 \\
9 / 12 / 94 \\
9 / 12 / 94 \\
9 / 12 / 94 \\
9 / 12 / 94 \\
9 / 12 / 94 \\
9 / 12 / 94 \\
9 / 12 / 94\end{array}$ & $\begin{array}{l}\text { L } \\
L \\
E \\
P \\
P \\
P \\
E \\
D \\
P \\
D\end{array}$ & $\begin{array}{l}0 \\
0 \\
.02 \\
.02 \\
.07 \\
.13 \\
.03 \\
0^{.37} \\
0\end{array}$ & $\begin{array}{l}7.8 \\
7.7 \\
7.2 \\
7.8 \\
8 \\
8 \\
7.5 \\
- \\
7.1 \\
--\end{array}$ & $\begin{array}{r}341 \\
312 \\
347 \\
1,441 \\
877 \\
878 \\
884 \\
-- \\
889 \\
--\end{array}$ & $\begin{array}{l}18.5 \\
17.0 \\
14.0 \\
19.0 \\
16.5 \\
17.0 \\
16.0 \\
-\overline{15.5} \\
--\end{array}$ & $\begin{array}{l}-- \\
8 \\
9.2 \\
-- \\
-- \\
-- \\
-- \\
-- \\
-- \\
--\end{array}$ \\
\hline $\begin{array}{l}3035 \\
3040 \\
3045 \\
3050 \\
3055 \\
3060 \\
3065 \\
3070 \\
3075 \\
3078\end{array}$ & $\begin{array}{l}\text { Seep } \\
\text { Stream } \\
\text { Stream } \\
\text { Stream } \\
\text { Seep } \\
\text { Stream } \\
\text { Seep } \\
\text { Stream } \\
\text { Seep } \\
\text { Seep }\end{array}$ & $\begin{array}{c}9 / 12 / 94 \\
9 / 12 / 94 \\
9 / 12 / 94 \\
9 / 12 / 94 \\
9 / 12 / 94 \\
9 / 12 / 94 \\
9 / 12 / 94 \\
9 / 12 / 94 \\
9 / 12 / 94 \\
--\end{array}$ & $\begin{array}{l}\text { L } \\
\text { D } \\
\text { D } \\
\text { D } \\
\text { D } \\
\text { D } \\
\text { D } \\
\text { D } \\
\text { L } \\
--\end{array}$ & $\begin{array}{l}0 \\
0 \\
0 \\
0 \\
0 \\
0 \\
0 \\
0 \\
0 \\
--\end{array}$ & $\begin{array}{l}7.7 \\
-- \\
-- \\
-- \\
-- \\
-- \\
-- \\
7.1 \\
--\end{array}$ & $\begin{array}{r}322 \\
- \\
- \\
- \\
- \\
- \\
- \\
- \\
389 \\
-\end{array}$ & $\begin{array}{l}19.0 \\
-- \\
-- \\
-- \\
-- \\
- \\
- \\
\overline{20.0} \\
--\end{array}$ & $\begin{array}{l}-- \\
- \\
-- \\
- \\
- \\
- \\
-- \\
- \\
-- \\
-\end{array}$ \\
\hline $\begin{array}{l}3080 \\
3085 \\
3090 \\
3095 \\
3100 \\
3105 \\
3110 \\
3115 \\
3120 \\
3125\end{array}$ & $\begin{array}{l}\text { Stream } \\
\text { Seep } \\
\text { Stream } \\
\text { Stream } \\
\text { Spring } \\
\text { Spring } \\
\text { Stream } \\
\text { Seep } \\
\text { Seep } \\
\text { Stream }\end{array}$ & $\begin{array}{c}9 / 12 / 94 \\
9 / 12 / 94 \\
9 / 13 / 94 \\
9 / 13 / 94 \\
9 / 13 / 94 \\
9 / 13 / 94 \\
-- \\
9 / 13 / 94 \\
9 / 13 / 94 \\
9 / 13 / 94\end{array}$ & $\begin{array}{l}\text { L } \\
D \\
\text { L } \\
\text { D } \\
\text { D } \\
\text { D } \\
-- \\
D \\
D \\
\text { E }\end{array}$ & $\begin{array}{l}0 \\
0 \\
0 \\
0 \\
0 \\
0 \\
- \\
0 \\
0 \\
.01\end{array}$ & $\begin{array}{l}7.2 \\
-- \\
6.9 \\
-- \\
-- \\
- \\
- \\
-- \\
- \\
6.9\end{array}$ & $\begin{array}{r}305 \\
-\overline{2} \\
452 \\
- \\
-- \\
-- \\
- \\
-- \\
\overline{100}\end{array}$ & $\begin{array}{l}21.0 \\
-\overline{17.5} \\
-- \\
-- \\
-- \\
-- \\
-- \\
- \\
17.5\end{array}$ & $\begin{array}{l}-- \\
-- \\
-- \\
-- \\
-- \\
-- \\
-- \\
-- \\
-- \\
--\end{array}$ \\
\hline
\end{tabular}


Table 5. Discharge and water-quality data for the low base flow seepage investigation at Bear Creek Valley, Oak Ridge, Tennessee, September 9 through September 29, 1994-Continued

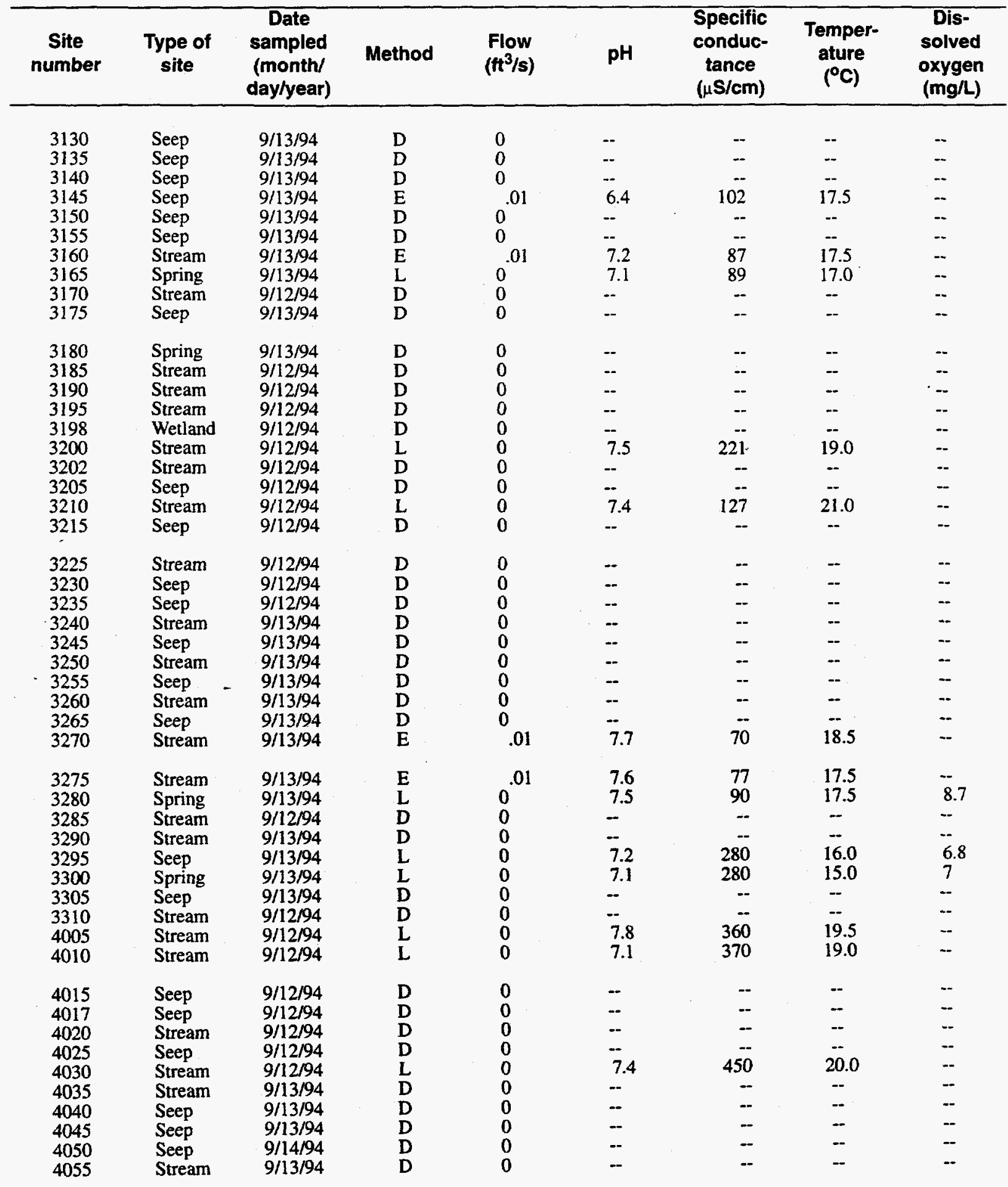


Table 5. Discharge and water-quality data for the low base flow seepage investigation at Bear Creek Valley, Oak Ridge, Tennessee, September 9 through September 29, 1994-Continued

\begin{tabular}{|c|c|c|c|c|c|c|c|c|}
\hline $\begin{array}{c}\text { Site } \\
\text { number }\end{array}$ & $\begin{array}{c}\text { Type of } \\
\text { site }\end{array}$ & $\begin{array}{c}\text { Date } \\
\text { sampled } \\
\text { (month/ } \\
\text { day/year) }\end{array}$ & Method & $\begin{array}{l}\text { Flow } \\
\left(f t^{3} / s\right)\end{array}$ & pH & $\begin{array}{c}\text { Specific } \\
\text { conduc- } \\
\text { tance } \\
(\mu \mathrm{S} / \mathrm{cm})\end{array}$ & $\begin{array}{c}\text { Temper- } \\
\text { ature } \\
\left({ }^{\circ} \mathrm{C}\right)\end{array}$ & $\begin{array}{c}\text { Dis- } \\
\text { solved } \\
\text { oxygen } \\
\text { (mg/l) }\end{array}$ \\
\hline 4060 & Seep & $9 / 13 / 94$ & D & 0 & -- & -- & -- & -- \\
\hline 4065 & Seep & $9 / 13 / 94$ & $\mathrm{D}$ & 0 & -- & -- & -- & -- \\
\hline 4070 & Seep & $9 / 13 / 94$ & D & 0 & -- & -- & - & -- \\
\hline 4075 & Spring & $9 / 13 / 94$ & D & 0 & -- & - & -- & -- \\
\hline 4080 & Stream & $9 / 13 / 94$ & D & 0 & -- & -- & -- & -- \\
\hline 4085 & Stream & $9 / 13 / 94$ & $\mathrm{D}$ & 0 & -- & -- & - & -- \\
\hline 4090 & Seep & $9 / 13 / 94$ & D & 0 & -- & -- & -- & -- \\
\hline 4095 & Seep & $9 / 13 / 94$ & D & 0 & -- & - & -- & -- \\
\hline 4100 & Stream & $9 / 13 / 94$ & D & 0 & -- & -- & - & -- \\
\hline 4105 & Spring & $9 / 13 / 94$ & D & 0 & -- & -- & - & -- \\
\hline 4110 & Stream & $9 / 12 / 94$ & $\mathrm{P}$ & .29 & 7.8 & 623 & 16.0 & -- \\
\hline 4115 & Spring & $9 / 13 / 94$ & $\mathbf{p}$ & .1 & 7 & 647 & 14.0 & 3.8 \\
\hline 4120 & Seep & $9 / 13 / 94$ & D & 0 & -- & - & -- & - \\
\hline 4125 & Stream & $9 / 13 / 94$ & $\bar{D}$ & 0 & - & -- & -- & -- \\
\hline 4400 & Stream & $9 / 12 / 94$ & $\mathbf{P}$ & .04 & 7.7 & 776 & 18.5 & - \\
\hline 4405 & Stream & $9 / 12 / 94$ & $P$ & .01 & 7.9 & 467 & 21.0 & -- \\
\hline 4410 & Stream & $9 / 12 / 94$ & $\mathrm{E}$ & .01 & 7.9 & 793 & 18.0 & -. \\
\hline 4415 & Seep & $9 / 12 / 94$ & $\mathbf{L}$ & 0 & -- & -- & $\ldots$ & -- \\
\hline 4420 & Spring & $9 / 12 / 94$ & E & .01 & 7.5 & 802 & 17.0 & 3.4 \\
\hline 4425 & Seep & $9 / 12 / 94$ & D & 0 & -- & -- & -- & -- \\
\hline 4430 & Stream & $9 / 12 / 94$ & $\mathbf{P}$ & .01 & 7.9 & 369 & 19.5 & -- \\
\hline 4435 & Stream & $9 / 12 / 94$ & $\mathbf{L}$ & 0 & 4.8 & 454 & 19.0 & -- \\
\hline 4440 & Stream & $9 / 13 / 94$ & $\bar{E}$ & .01 & 8 & 576 & 15.5 & -- \\
\hline 4445 & Seep & $9 / 13 / 94$ & D & 0 & - & -. & - & - \\
\hline 4450 & Stream & $9 / 13 / 94$ & D & 0 & - & -- & $\ddot{z}$ & -- \\
\hline 4455 & Stream & $9 / 13 / 94$ & $\mathbf{E}$ & .01 & 7.7 & 725 & 17.0 & - \\
\hline 4472 & Seep & $9 / 13 / 94$ & D & 0 & -- & - & - & - \\
\hline 4473 & Spring & $9 / 13 / 94$ & L & 0 & 7.2 & 94 & 18.5 & -- \\
\hline 4475 & Stream & $9 / 12 / 94$ & $\overline{\mathbf{P}}$ & .02 & 7.7 & 326 & 19.5 & -- \\
\hline 4480 & Stream & $9 / 13 / 94$ & $\mathbf{P}$ & .01 & 7.6 & 194 & 17.0 & -- \\
\hline 4485 & Stream & $9 / 13 / 94$ & $\mathbf{E}$ & .01 & 7.7 & 193 & 17.0 & -- \\
\hline 4490 & Stream & $9 / 13 / 94$ & $\mathbf{L}$ & 0 & 7 & 395 & 16.0 & $\cdots$ \\
\hline 4495 & Seep & $9 / 13 / 94$ & $\overline{\mathrm{D}}$ & 0 & -- & -- & $=$ & -- \\
\hline 4500 & Stream & $9 / 13 / 94$ & $\mathbf{L}$ & 0 & 7.4 & 90 & 17.0 & -- \\
\hline 4505 & Seep & $9 / 13 / 94$ & $\bar{D}$ & 0 & - & -- & $\ddot{-}$ & -- \\
\hline 4510 & Seep & $9 / 13 / 94$ & $\mathbf{L}$ & 0 & 6.4 & 142 & 18.5 & -- \\
\hline 4515 & Spring & $9 / 13 / 94$ & $\mathbf{L}$ & 0 & 6.8 & 90 & 17.0 & 8.6 \\
\hline 4520 & Stream & $9 / 12 / 94$ & $\overline{\mathbf{D}}$ & 0 & -- & - & -- & -- \\
\hline 4525 & Stream & $9 / 12 / 94$ & D & 0 & -- & $\ddot{-}$ & -- & -- \\
\hline 4530 & Stream & $9 / 12 / 94$ & $\mathbf{P}$ & .08 & 8 & 837 & 18.0 & -- \\
\hline 4535 & Stream & $9 / 13 / 94$ & D & 0 & -. & -- & -. & -- \\
\hline 4540 & Stream & $9 / 13 / 94$ & $\bar{D}$ & 0 & -. & -- & - & -- \\
\hline 4545 & Stream & $9 / 13 / 94$ & D & 0 & -- & - & -- & -- \\
\hline 4580 & Stream & $9 / 13 / 94$ & D & 0 & -- & -- & -- & $\cdots$ \\
\hline 4585 & Stream & $9 / 14 / 94$ & D & 0 & - & -- & -- & -- \\
\hline 4590 & Stream & $9 / 13 / 94$ & D & 0 & - & -- & -- & -- \\
\hline 4595 & Stream & $9 / 13 / 94$ & D & 0 & -- & -- & $\cdots$ & -- \\
\hline 4600 & Strearn & $9 / 13 / 94$ & $\mathrm{D}$ & 0 & -- & -- & -- & -- \\
\hline 4605 & Seep & $9 / 13 / 94$ & D & 0 & -- & - & - & -- \\
\hline 4610 & Stream & $9 / 13 / 94$ & D & 0 & -- & -- & -- & - \\
\hline
\end{tabular}


Table 5. Discharge and water-quality data for the low base flow seepage investigation at Bear Creek Valley, Oak Ridge, Tennessee, September 9 through September 29, 1994-Continued

\begin{tabular}{|c|c|c|c|c|c|c|c|c|}
\hline $\begin{array}{c}\text { Site } \\
\text { number }\end{array}$ & $\begin{array}{l}\text { Type of } \\
\text { site }\end{array}$ & $\begin{array}{c}\text { Date } \\
\text { sampled } \\
\text { (month/ } \\
\text { day/year) }\end{array}$ & Method & $\begin{array}{l}\text { Flow } \\
\left(\mathrm{ft}^{3} / \mathrm{s}\right)\end{array}$ & pH & $\begin{array}{c}\text { Specific } \\
\text { conduc- } \\
\text { tance } \\
(\mu \mathrm{S} / \mathrm{cm})\end{array}$ & $\begin{array}{c}\text { Temper- } \\
\text { ature } \\
\left({ }^{\circ} \mathrm{C}\right)\end{array}$ & $\begin{array}{c}\text { Dis- } \\
\text { solved } \\
\text { oxygen } \\
\text { (mg/L) }\end{array}$ \\
\hline 4615 & Seep & $9 / 13 / 94$ & D & 0 & -. & -. & -- & -- \\
\hline 4620 & Spring & $9 / 13 / 94$ & $\bar{D}$ & 0 & -- & -- & - & -- \\
\hline 4630 & Stream & $9 / 12 / 94$ & $\mathrm{P}$ & .07 & 7.9 & 879 & 16.5 & -- \\
\hline 5005 & Stream & $9 / 12 / 94$ & $\mathbf{P}$ & .2 & 8.2 & 597 & 18.5 & -- \\
\hline 5010 & Stream & $9 / 14 / 94$ & D & 0 & -- & -. & - & -- \\
\hline 5015 & Stream & $9 / 13 / 94$ & D & 0 & -- & -- & - & -- \\
\hline 5020 & Stream & $9 / 13 / 94$ & D & 0 & -- & -- & -- & -- \\
\hline 5025 & Seep & $9 / 13 / 94$ & $\bar{D}$ & 0 & -- & -- & - & -- \\
\hline 5030 & Stream & $9 / 12 / 94$ & $\mathrm{P}$ & .26 & 8.2 & 602 & 18.5 & - \\
\hline 5035 & Stream & $9 / 13 / 94$ & D & 0 & -- & -- & -- & -- \\
\hline 5040 & Seep & $9 / 13 / 94$ & D & 0 & -- & - & -- & - \\
\hline 5045 & Stream & $9 / 13 / 94$ & D & 0 & -- & - & -- & - \\
\hline 5050 & Stream & $9 / 14 / 94$ & D & 0 & -. & -- & -. & - \\
\hline 5055 & Stream & $9 / 13 / 94$ & D & 0 & -- & -- & -- & -- \\
\hline 5060 & Seep & $9 / 13 / 94$ & D & 0 & - & - & -- & -- \\
\hline 5065 & Seep & $9 / 13 / 94$ & D & 0 & -- & - & -- & - \\
\hline 5070 & Seep & $9 / 13 / 94$ & D & 0 & -- & - & -- & -- \\
\hline 5075 & Stream & $9 / 13 / 94$ & D & 0 & -- & -- & -- & -- \\
\hline 5080 & Seep & $9 / 13 / 94$ & D & 0 & -- & -- & -- & -- \\
\hline 5085 & Seep & $9 / 13 / 94$ & D & 0 & - & - & - & -- \\
\hline 5090 & Stream & $9 / 13 / 94$ & D & 0 & -- & -- & - & -- \\
\hline 5095 & Stream & $9 / 13 / 94$ & D & 0 & -- & -- & -- & -- \\
\hline 5100 & Seep & $9 / 13 / 94$ & D & 0 & -- & $\ddot{-}$ & -- & -- \\
\hline 5105 & Stream & $9 / 12 / 94$ & $\mathbf{P}$ & .22 & 8.1 & 613 & 17.0 & -- \\
\hline 6005 & Stream & 9/14/94 & $\mathbf{F}$ & .03 & 7.8 & 258 & 17.5 & -- \\
\hline 6007 & Stream & $9 / 14 / 94$ & D & 0 & - & - & - & -- \\
\hline 6010 & Stream & $9 / 13 / 94$ & $E$ & .01 & 8 & 232 & 18.5 & -- \\
\hline 6015 & Seep & $9 / 13 / 94$ & $\bar{D}$ & 0 & -- & -. & -- & -- \\
\hline 6020 & Seep & $9 / 13 / 94$ & D & 0 & $\ddot{z}$ & $\ddot{-}$ & - & - \\
\hline 6025 & Stream & $9 / 13 / 94$ & $\mathbf{E}$ & 0.01 & 7.2 & 132 & 17.5 & - \\
\hline 6030 & Stream & $9 / 13 / 94$ & $\mathbf{E}$ & .01 & 7.4 & 119 & 17.0 & -- \\
\hline 6033 & Seep & $9 / 13 / 94$ & D & 0 & - & - & 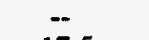 & - \\
\hline 6035 & Stream & $9 / 13 / 94$ & $\mathbf{E}$ & .01 & 7.8 & 101 & 17.5 & $\overline{0}$ \\
\hline 6045 & Seep & $9 / 13 / 94$ & $\overline{\mathbf{E}}$ & .01 & 7.3 & 83 & 16.0 & 9 \\
\hline 6050 & Stream & $9 / 13 / 94$ & D & 0 & -- & - & -- & -- \\
\hline 6055 & Seep & $9 / 13 / 94$ & D & 0 & -- & -- & $\because$ & -- \\
\hline 6060 & Stream & $9 / 13 / 94$ & $\mathbf{p}$ & .2 & 8 & 591 & 16.5 & - \\
\hline 6065 & Stream & $9 / 13 / 94$ & $\mathbf{P}$ & .19 & -- & -- & -- & -- \\
\hline 6070 & Stream & $9 / 13 / 94$ & D & 0 & -- & -- & -- & -- \\
\hline 6075 & Stream & $9 / 13 / 94$ & D & 0 & - & - & - & -- \\
\hline 6085 & Seep & $9 / 13 / 94$ & D & 0 & -- & - & - & -- \\
\hline 6090 & Stream & $9 / 13 / 94$ & D & 0 & - & - & $\cdots$ & -- \\
\hline 6095 & Seep & $9 / 13 / 94$ & D & 0 & $\bar{z}$ & $\ddot{n}$ & $\ddot{105}$ & -- \\
\hline 6100 & Stream & $9 / 13 / 94$ & $\mathbf{L}$ & 0 & 7.9 & 299 & 18.5 & - \\
\hline 6105 & Seep & $9 / 13 / 94$ & D & 0 & -- & -- & - & -- \\
\hline 6110 & Seep & $9 / 13 / 94$ & D & 0 & - & $\ddot{-}$ & 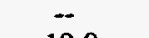 & -- \\
\hline 6115 & Stream & $9 / 13 / 94$ & $\mathbf{E}$ & .01 & 7.5 & 90 & 19.0 & -- \\
\hline 6120 & Spring & $9 / 13 / 94$ & $\mathbf{L}$ & 0 & 7 & 131 & 17.5 & 9.1 \\
\hline 7005 & Stream & $9 / 13 / 94$ & $\mathbf{P}$ & .21 & 8.2 & 613 & 15.5 & - \\
\hline 7010 & Stream & $9 / 13 / 94$ & D & 0 & -- & - & - & -- \\
\hline
\end{tabular}


Table 5. Discharge and water-quality data for the low base flow seepage investigation at Bear Creek Valley,

Oak Ridge, Tennessee, September 9 through September 29, 1994-Continued

\begin{tabular}{|c|c|c|c|c|c|c|c|c|}
\hline $\begin{array}{c}\text { Site } \\
\text { number }\end{array}$ & $\begin{array}{l}\text { Type of } \\
\text { site }\end{array}$ & $\begin{array}{c}\text { Date } \\
\text { sampled } \\
\text { (month/ } \\
\text { day/year) }\end{array}$ & Method & $\begin{array}{l}\text { Flow } \\
\left(\mathrm{ft}^{3} / \mathrm{s}\right)\end{array}$ & pH & $\begin{array}{l}\text { Specific } \\
\text { conduc- } \\
\text { tance } \\
(\mu \mathrm{S} / \mathrm{cm})\end{array}$ & $\begin{array}{c}\text { Temper- } \\
\text { ature } \\
\left({ }^{\circ} \mathrm{C}\right)\end{array}$ & $\begin{array}{c}\text { Dis- } \\
\text { solved } \\
\text { oxygen } \\
\text { (mg/l) }\end{array}$ \\
\hline $\begin{array}{l}7015 \\
7020 \\
7025 \\
7030 \\
7035 \\
7040 \\
7045 \\
7050 \\
7055 \\
7060\end{array}$ & $\begin{array}{l}\text { Stream } \\
\text { Spring } \\
\text { Stream } \\
\text { Stream } \\
\text { Stream } \\
\text { Wetland } \\
\text { Stream } \\
\text { Seep } \\
\text { Stream } \\
\text { Stream }\end{array}$ & $\begin{array}{l}9 / 13 / 94 \\
9 / 13 / 94 \\
9 / 13 / 94 \\
9 / 13 / 94 \\
9 / 13 / 94 \\
9 / 13 / 94 \\
9 / 13 / 94 \\
9 / 13 / 94 \\
9 / 13 / 94 \\
9 / 12 / 94\end{array}$ & $\begin{array}{l}E \\
E \\
E \\
E \\
D \\
D \\
D \\
D \\
D \\
P\end{array}$ & $\begin{array}{l}.01 \\
.01 \\
.01 \\
.01 \\
0 \\
0 \\
0 \\
0 \\
0 \\
.27\end{array}$ & $\begin{array}{l}7.8 \\
6.8 \\
7.6 \\
7.6 \\
-- \\
-- \\
-- \\
-- \\
- \\
8.2\end{array}$ & $\begin{array}{r}238 \\
272 \\
212 \\
206 \\
-. \\
-- \\
-- \\
-- \\
-- \\
593\end{array}$ & $\begin{array}{l}17.0 \\
16.5 \\
17.5 \\
17.0 \\
-- \\
-- \\
-- \\
-- \\
-- \\
19.5\end{array}$ & $\begin{array}{l}-- \\
6.1 \\
-- \\
-- \\
-- \\
-- \\
-- \\
-- \\
-- \\
--\end{array}$ \\
\hline $\begin{array}{l}8005 \\
8010 \\
8015 \\
8020 \\
8025 \\
8030 \\
8035 \\
8040 \\
8045 \\
8050\end{array}$ & $\begin{array}{l}\text { Stream } \\
\text { Seep } \\
\text { Seep } \\
\text { Stream } \\
\text { Stream } \\
\text { Stream } \\
\text { Stream } \\
\text { Seep } \\
\text { Seep } \\
\text { Seep }\end{array}$ & $\begin{array}{l}9 / 13 / 94 \\
9 / 13 / 94 \\
9 / 13 / 94 \\
9 / 13 / 94 \\
9 / 13 / 94 \\
9 / 13 / 94 \\
9 / 13 / 94 \\
9 / 13 / 94 \\
9 / 13 / 94 \\
9 / 13 / 94\end{array}$ & $\begin{array}{l}\text { P } \\
\text { D } \\
D \\
\text { P } \\
D \\
D \\
D \\
D \\
D \\
D\end{array}$ & $\begin{array}{l}.02 \\
0 \\
0 \\
.05 \\
0 \\
0 \\
0 \\
0 \\
0 \\
0\end{array}$ & $\begin{array}{l}8.1 \\
- \\
-- \\
8.3 \\
-- \\
-- \\
-- \\
-- \\
-- \\
--\end{array}$ & $\begin{array}{r}238 \\
-- \\
-- \\
187 \\
-- \\
-- \\
- \\
- \\
-- \\
--\end{array}$ & $\begin{array}{l}16.0 \\
- \\
-- \\
15.5 \\
-- \\
-- \\
-- \\
-- \\
--\end{array}$ & $\begin{array}{l}8 \\
-- \\
-- \\
8.5 \\
-- \\
-- \\
-- \\
-- \\
-- \\
--\end{array}$ \\
\hline $\begin{array}{l}8055 \\
8060 \\
8062 \\
8063 \\
8065 \\
8070 \\
8075 \\
8080 \\
8085 \\
8090\end{array}$ & $\begin{array}{l}\text { Stream } \\
\text { Seep } \\
\text { Stream } \\
\text { Seep } \\
\text { Stream } \\
\text { Stream } \\
\text { Seep } \\
\text { Stream } \\
\text { Seep } \\
\text { Stream }\end{array}$ & $\begin{array}{l}9 / 13 / 94 \\
9 / 13 / 94 \\
9 / 13 / 94 \\
9 / 13 / 94 \\
9 / 13 / 94 \\
9 / 13 / 94 \\
9 / 13 / 94 \\
9 / 13 / 94 \\
9 / 13 / 94 \\
9 / 13 / 94\end{array}$ & $\begin{array}{l}\text { F } \\
\text { D } \\
\text { F } \\
\text { D } \\
\text { D } \\
\text { D } \\
\text { D } \\
\text { D } \\
\text { D } \\
\text { F }\end{array}$ & $\begin{array}{l}.01 \\
0 \\
0 \\
0 \\
0 \\
0 \\
0 \\
0 \\
0 \\
0\end{array}$ & $\begin{array}{l}8.3 \\
-- \\
8.1 \\
-- \\
-- \\
-- \\
-- \\
-- \\
\overline{7} .1\end{array}$ & $\begin{array}{r}184 \\
-\ddot{2} \\
227 \\
-- \\
-- \\
-- \\
- \\
- \\
\overline{74}\end{array}$ & $\begin{array}{l}16.0 \\
-- \\
16.5 \\
-- \\
-- \\
-- \\
-- \\
-- \\
-\overline{17.0}\end{array}$ & $\begin{array}{l}8.7 \\
\overline{7.5} \\
-- \\
- \\
-- \\
-- \\
-- \\
-- \\
3.9\end{array}$ \\
\hline $\begin{array}{l}8092 \\
8095 \\
8100 \\
8103 \\
8105 \\
8110 \\
8115 \\
8120 \\
8125 \\
8130\end{array}$ & $\begin{array}{l}\text { Seep } \\
\text { Stream } \\
\text { Seep } \\
\text { Stream } \\
\text { Stream } \\
\text { Seep } \\
\text { Stream } \\
\text { Seep } \\
\text { Stream } \\
\text { Seep }\end{array}$ & $\begin{array}{l}9 / 13 / 94 \\
9 / 13 / 94 \\
9 / 13 / 94 \\
9 / 13 / 94 \\
9 / 13 / 94 \\
9 / 13 / 94 \\
9 / 13 / 94 \\
9 / 13 / 94 \\
9 / 13 / 94 \\
9 / 13 / 94\end{array}$ & $\begin{array}{l}\text { D } \\
\text { D } \\
\text { D } \\
\text { L } \\
\text { D } \\
\text { D } \\
\text { D } \\
\text { L } \\
\text { L } \\
\text { D }\end{array}$ & $\begin{array}{l}0 \\
0 \\
0 \\
0 \\
0 \\
0 \\
0 \\
0 \\
0 \\
0\end{array}$ & $\begin{array}{l}-- \\
-- \\
6.2 \\
-- \\
-- \\
-- \\
6.1 \\
6.2 \\
--\end{array}$ & $\begin{array}{c}- \\
-- \\
\overline{47} \\
-- \\
-- \\
-- \\
36 \\
48 \\
--\end{array}$ & $\begin{array}{l}-- \\
-- \\
-\overline{17.5} \\
-- \\
-- \\
-- \\
18.5 \\
17.0 \\
--\end{array}$ & $\begin{array}{l}- \\
- \\
-- \\
4.4 \\
- \\
-- \\
-- \\
-- \\
7.8 \\
--\end{array}$ \\
\hline $\begin{array}{l}8135 \\
8140 \\
8145 \\
8150 \\
8200 \\
8205 \\
8210 \\
8220 \\
8225 \\
9005\end{array}$ & $\begin{array}{l}\text { Stream } \\
\text { Seep } \\
\text { Seep } \\
\text { Seep } \\
\text { Seep } \\
\text { Seep } \\
\text { Stream } \\
\text { Seep } \\
\text { Seep } \\
\text { Stream }\end{array}$ & $\begin{array}{l}9 / 13 / 94 \\
9 / 13 / 94 \\
9 / 13 / 94 \\
9 / 13 / 94 \\
9 / 13 / 94 \\
9 / 13 / 94 \\
9 / 13 / 94 \\
9 / 13 / 94 \\
9 / 13 / 94 \\
9 / 13 / 94\end{array}$ & $\begin{array}{l}\text { D } \\
\text { D } \\
\text { D } \\
\text { D } \\
\text { D } \\
\text { D } \\
\text { D } \\
\text { D } \\
\text { D } \\
\text { P }\end{array}$ & $\begin{array}{l}0 \\
0 \\
0 \\
0 \\
0 \\
0 \\
0 \\
0 \\
0 \\
.24\end{array}$ & $\begin{array}{l}- \\
-- \\
-- \\
-- \\
-- \\
- \\
-- \\
-- \\
- \\
8\end{array}$ & $\begin{array}{l}-- \\
-- \\
-- \\
-- \\
- \\
-- \\
-- \\
- \\
- \\
539\end{array}$ & $\begin{array}{l}-- \\
-- \\
-- \\
-- \\
-- \\
-- \\
-- \\
\overline{18.5}\end{array}$ & $\begin{array}{l}- \\
-- \\
- \\
- \\
- \\
- \\
- \\
- \\
- \\
-\end{array}$ \\
\hline
\end{tabular}


Table 5. Discharge and water-quality data for the low base flow seepage investigation at Bear Creek Valley, Oak Ridge, Tennessee, September 9 through September 29, 1994-Continued

\begin{tabular}{|c|c|c|c|c|c|c|c|c|}
\hline $\begin{array}{c}\text { Site } \\
\text { number }\end{array}$ & $\begin{array}{l}\text { Type of } \\
\text { site }\end{array}$ & $\begin{array}{c}\text { Date } \\
\text { sampled } \\
\text { (month/ } \\
\text { day/year) }\end{array}$ & Method & $\begin{array}{l}\text { Flow } \\
\left(\mathrm{ft}^{3} / \mathrm{s}\right)\end{array}$ & $\mathrm{pH}$ & $\begin{array}{c}\text { Specific } \\
\text { conduc- } \\
\text { tance } \\
(\mu \mathrm{S} / \mathrm{cm})\end{array}$ & $\begin{array}{l}\text { Temper- } \\
\text { ature } \\
\left({ }^{\circ} \mathrm{C}\right)\end{array}$ & $\begin{array}{c}\text { Dis- } \\
\text { solved } \\
\text { oxygen } \\
\text { (mg/L) }\end{array}$ \\
\hline 9010 & Stream & $9 / 13 / 94$ & $P$ & .05 & 8.2 & 282 & 18.5 & -- \\
\hline 9015 & Stream & $9 / 13 / 94$ & $\mathbf{L}$ & 0 & 7.9 & 259 & 19.0 & -- \\
\hline 9020 & Stream & $9 / 13 / 94$ & D & 0 & -- & - & - & -- \\
\hline 9025 & Seep & $9 / 13 / 94$ & D & 0 & -. & - & -- & -. \\
\hline 9030 & Stream & $9 / 13 / 94$ & D & 0 & -- & - & -- & -. \\
\hline 9035 & Seep & $9 / 13 / 94$ & D & 0 & -- & -- & - & -. \\
\hline 9040 & Stream & $9 / 13 / 94$ & $\mathrm{~L}$ & 0 & 7.9 & 251 & 19.0 & -- \\
\hline 9045 & Seep & $9 / 13 / 94$ & $\mathrm{D}$ & 0 & -- & - & - & -- \\
\hline 9050 & Stream & $9 / 13 / 94$ & $\bar{L}$ & 0 & 7.8 & 188 & 18.5 & -- \\
\hline 9055 & Stream & $9 / 14 / 94$ & D & 0 & -- & - & - & -- \\
\hline 9060 & Seep & $9 / 14 / 94$ & $D$ & 0 & -- & -- & -- & - \\
\hline 9065 & Stream & $9 / 14 / 94$ & $\mathrm{~L}$ & 0 & 7.8 & 234 & 17.5 & -- \\
\hline 9068 & Seep & $9 / 14 / 94$ & D & 0 & -. & -- & -- & -- \\
\hline 9070 & Seep & $9 / 14 / 94$ & D & 0 & -- & -- & -- & -- \\
\hline 9075 & Stream & $9 / 14 / 94$ & D & 0 & -- & -. & -- & -- \\
\hline 9080 & Stream & $9 / 14 / 94$ & $\mathrm{D}$ & 0 & -- & -- & -- & -- \\
\hline 9085 & Seep & $9 / 14 / 94$ & D & 0 & - & -- & - & -- \\
\hline 9090 & Stream & $9 / 13 / 94$ & $\overrightarrow{\mathbf{P}}$ & .23 & 8 & 545 & 16.5 & -- \\
\hline 9092 & Stream & $9 / 14 / 94$ & $\mathbf{E}$ & .01 & 7.6 & 401 & 13.5 & -- \\
\hline 9093 & Spring & $9 / 14 / 94$ & D & 0 & $\cdots$ & -- & - & -- \\
\hline 9095 & Stream & $9 / 13 / 94$ & $\mathbf{P}$ & .21 & 7.8 & 577 & 16.5 & -- \\
\hline 10005 & Stream & $9 / 13 / 94$ & $\mathbf{P}$ & .09 & 8 & 275 & 18.0 & -- \\
\hline 10070 & Stream & $9 / 13 / 94$ & D & 0 & -. & -- & - & -- \\
\hline 10080 & Stream & $9 / 13 / 94$ & D & 0 & -- & -- & -- & -- \\
\hline 10090 & Stream & $9 / 13 / 94$ & D & 0 & -- & -- & -- & - \\
\hline 10095 & Seep & $9 / 13 / 94$ & $\bar{D}$ & 0 & -- & - & -- & -- \\
\hline 10100 & Seep & $9 / 13 / 94$ & D & 0 & -- & - & -- & - \\
\hline 11005 & Stream & $9 / 14 / 94$ & $\bar{D}$ & 0 &.- & - & -- & -- \\
\hline 11010 & Stream & $9 / 14 / 94$ & D & 0 & -- & - & - & -- \\
\hline 11015 & Seep & $9 / 14 / 94$ & D & 0 & -- & - & - & - \\
\hline 11020 & Stream & $9 / 14 / 94$ & $\mathbf{P}$ & .01 & 7.3 & 246 & 16.5 & -- \\
\hline 11025 & Stream & $9 / 14 / 94$ & D & 0 & -- & - & - & -- \\
\hline 11030 & Stream & $9 / 14 / 94$ & D & 0 & - & - & $\bar{n}$ & $\bar{\varepsilon}$ \\
\hline 11035 & Seep & $9 / 14 / 94$ & L & 0 & 6 & 39 & 17.0 & 5.6 \\
\hline 11040 & Stream & $9 / 14 / 94$ & D & 0 & - & - & - & - \\
\hline 11045 & Spring & $9 / 14 / 94$ & $\bar{D}$ & 0 & -- & -- & - & - \\
\hline 11050 & Stream & $9 / 14 / 94$ & $\overline{\mathbf{D}}$ & 0 & -- & -- & -- & -- \\
\hline 11055 & Stream & $9 / 13 / 94$ & $\mathbf{P}$ & .2 & 8.2 & 463 & 18.5 & - \\
\hline 11060 & Stream & $9 / 13 / 94$ & $\mathbf{P}$ & .32 & 8.1 & 463 & 18.5 & -- \\
\hline 11065 & Stream & $9 / 14 / 94$ & $\mathrm{D}$ & 0 & -- & - & $\cdots$ & - \\
\hline 11070 & Stream & $9 / 14 / 94$ & $\mathbf{L}$ & 0 & 7.2 & 271 & 17.5 & 3.6 \\
\hline 11075 & Seep & $9 / 14 / 94$ & $\vec{D}$ & 0 & -- & - & -- & -- \\
\hline 11080 & Stream & $9 / 14 / 94$ & $\mathbf{L}$ & 0 & 7.4 & 243 & 19.5 & -- \\
\hline 11085 & Spring & $9 / 14 / 94$ & $\overline{\mathrm{D}}$ & 0 & -- & -- & -- & -- \\
\hline 11090 & Stream & $9 / 14 / 94$ & L & 0 & 7.4 & 332 & 19.0 & -- \\
\hline 11095 & Spring & $9 / 14 / 94$ & $\mathbf{L}$ & 0 & 7.2 & 208 & 18.5 & 5.6 \\
\hline 11099 & Spring & $9 / 14 / 94$ & L & 0 & 7.3 & 371 & 15.0 & 8.3 \\
\hline 11100 & Stream & $9 / 13 / 94$ & $\mathbf{P}$ & .26 & 7.8 & 467 & 19.5 & - \\
\hline 11105 & Stream & $9 / 13 / 94$ & $\mathbf{P}$ & .34 & 7.8 & 472 & 19.0 & -- \\
\hline 12010 & Stream & $9 / 14 / 94$ & $\mathbf{P}$ & .1 & 7.8 & 289 & 14.5 & - \\
\hline
\end{tabular}


Table 5. Discharge and water-quality data for the low base flow seepage investigation at Bear Creek Valley, Oak Ridge, Tennessee, September 9 through September 29, 1994-Continued

\begin{tabular}{|c|c|c|c|c|c|c|c|c|}
\hline $\begin{array}{c}\text { Site } \\
\text { number }\end{array}$ & $\begin{array}{l}\text { Type of } \\
\text { site }\end{array}$ & $\begin{array}{c}\text { Date } \\
\text { sampled } \\
\text { (month/ } \\
\text { day/year) }\end{array}$ & Method & $\begin{array}{l}\text { Flow } \\
\left(\mathrm{ft}^{3} / \mathrm{s}\right)\end{array}$ & pH & $\begin{array}{c}\text { Specific } \\
\text { conduc- } \\
\text { tance } \\
(\mu \mathrm{S} / \mathrm{cm})\end{array}$ & $\begin{array}{c}\text { Temper- } \\
\text { ature } \\
\left({ }^{\circ} \mathrm{C}\right)\end{array}$ & $\begin{array}{c}\text { Dis- } \\
\text { solved } \\
\text { oxygen } \\
\text { (mg/L) }\end{array}$ \\
\hline 12015 & Seep & $9 / 14 / 94$ & $\mathrm{D}$ & 0 & -- & -- & -- & -- \\
\hline 12020 & Seep & $9 / 14 / 94$ & $\bar{L}$ & 0 & 7.5 & 386 & 17.5 & -- \\
\hline 12025 & Spring & $9 / 14 / 94$ & $\mathrm{E}$ & .1 & 7.4 & 290 & 14.0 & 7.8 \\
\hline 12040 & Stream & $9 / 14 / 94$ & $\mathrm{D}$ & 0 & -- & - & -- & - \\
\hline 12045 & Stream & $9 / 14 / 94$ & D & 0 & -- & -- & - & -- \\
\hline 12050 & Seep & $9 / 14 / 94$ & $\bar{D}$ & 0 & -- & -- & -- & -- \\
\hline 13005 & Stream & $9 / 14 / 94$ & $\mathbf{P}$ & .6 & 8.1 & 395 & 19.0 & -- \\
\hline 13007 & Stream & $9 / 15 / 94$ & D & 0 & -- & -- & -- & -- \\
\hline 13008 & Seep & $9 / 15 / 94$ & D & 0 & -- & -- & - & -- \\
\hline 13010 & Seep & $9 / 15 / 94$ & D & 0 & -- & - & - & -- \\
\hline 13015 & Seep & $9 / 15 / 94$ & D & 0 & - & -- & -. & -- \\
\hline 13020 & Spring & $9 / 15 / 94$ & D & 0 & -- & - & - & - \\
\hline 13025 & Stream & $9 / 14 / 94$ & $\mathbf{P}$ & .42 & 7.9 & 404 & 16.5 & -- \\
\hline 13030 & Stream & $9 / 15 / 94$ & D & 0 & - & -- & -- & -- \\
\hline 13035 & Seep & $9 / 15 / 94$ & D & 0 & - & -- & -- & -- \\
\hline 13040 & Seep & $9 / 15 / 94$ & D & 0 & -- & -- & -- & -- \\
\hline 13060 & Stream & $9 / 15 / 94$ & D & 0 & -- & -- & -- & -- \\
\hline 13070 & Stream & $9 / 15 / 94$ & D & 0 & -- & -- & -- & -- \\
\hline 13075 & Stream & $9 / 15 / 94$ & D & 0 & -- & -- & -- & -- \\
\hline 13080 & Seep & $9 / 15 / 94$ & D & 0 & -- & -- & -- & - \\
\hline 13085 & Stream & $9 / 15 / 94$ & D & 0 & - & -- & -- & -- \\
\hline 13086 & Seep & $9 / 15 / 94$ & D & 0 & - & -- & -- & -- \\
\hline 13087 & Stream & $9 / 15 / 94$ & D & 0 & -- & -- & -- & -- \\
\hline 13088 & Stream & $9 / 15 / 94$ & D & 0 & - & -- & - & -- \\
\hline 13089 & Seep & 9/15/94 & D & 0 & -- & -- & - & -- \\
\hline 13090 & Stream & - 9/15/94 & D & 0 & -- & -- & $\cdots$ & -- \\
\hline 13095 & Seep & $-\quad 9 / 15 / 94$ & D & 0 & -- & - & -- & -- \\
\hline 13140 & Stream & $9 / 15 / 94$ & D & 0 & - & - & -- & - \\
\hline 13150 & Seep & $9 / 15 / 94$ & D & 0 & $\overline{-}$ & - & $\ddot{10}$ & - \\
\hline 13155 & Stream & $9 / 15 / 94$ & L & 0 & 7.4 & 63 & 18.0 & - \\
\hline 13160 & Stream & $9 / 15 / 94$ & D & 0 & -. & - & -- & -- \\
\hline 13165 & Seep & $9 / 15 / 94$ & D & 0 & $\bar{z}$ & -- & 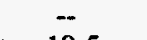 & -- \\
\hline 13170 & Stream & $9 / 15 / 94$ & $\mathbf{L}$ & 0 & 7.5 & 70 & 19.5 & -- \\
\hline 13175 & Spring & $9 / 15 / 94$ & $\mathbf{L}$ & 0 & 7.6 & 90 & 17.5 & 7.5 \\
\hline 13180 & Stream & $9 / 15 / 94$ & D. & 0 & 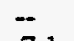 & 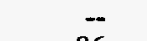 & - & $\ddot{7}$ \\
\hline 13200 & Spring & $9 / 15 / 94$ & $\mathbf{L}$ & 0 & 7.1 & 86 & 20.0 & 7.8 \\
\hline 13205 & Stream & $9 / 14 / 94$ & D & 0 & - & -- & -- & - \\
\hline 13210 & Stream & $9 / 14 / 94$ & D & 0 & - & -- & - & -- \\
\hline 13215 & Seep & $9 / 14 / 94$ & D & 0 & -- & $\cdots$ & -- & $\cdots$ \\
\hline 13220 & Seep & $9 / 14 / 94$ & D & 0 & -- & - & - & $\cdots$ \\
\hline 13225 & Stream & $9 / 14 / 94$ & D & 0 & -- & 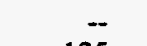 & $-\overline{0}$ & -- \\
\hline 13230 & Stream & $9 / 14 / 94$ & $\mathbf{L}$ & 0 & 7.2 & 135 & 20.5 & $=$ \\
\hline 13235 & Spring & $9 / 14 / 94$ & $\mathbf{L}$ & 0 & 6.9 & 123 & 15.0 & 7.6 \\
\hline 13240 & Seep & $9 / 14 / 94$ & D & 0 & -- & -- & -- & $-\cdot$ \\
\hline 13245 & Stream & $9 / 14 / 94$ & D & 0 & -- & -- & -- & -- \\
\hline 13250 & Seep & $9 / 14 / 94$ & D & 0 & -- & -- & -- & -- \\
\hline 13255 & Stream & $9 / 14 / 94$ & D & 0 & -- & -- & -- & -- \\
\hline 13260 & Stream & $9 / 14 / 94$ & D & 0 & $-\cdot$ & $\cdots$ & -- & -- \\
\hline 13265 & Seep & $9 / 14 / 94$ & D & 0 & -- & - & -- & -- \\
\hline 13270 & Stream & $9 / 14 / 94$ & D & 0 & -- & -- & -- & -- \\
\hline
\end{tabular}


Table 5. Discharge and water-quality data for the low base flow seepage investigation at Bear Creek Valley, Oak Ridge, Tennessee, September 9 through September 29, 1994-Continued

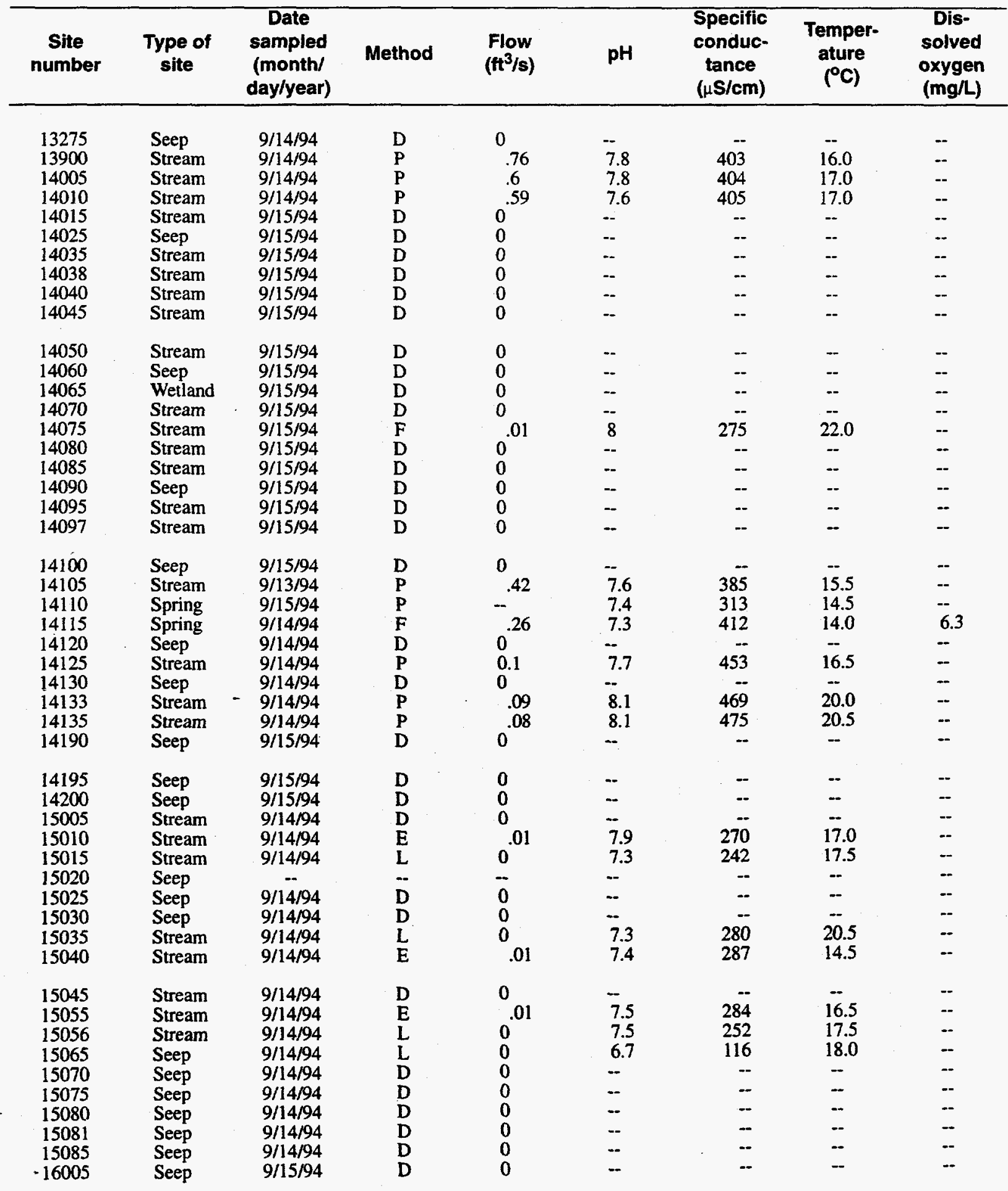


Table 5. Discharge and water-quality data for the low base flow seepage investigation at Bear Creek Valley, Oak Ridge, Tennessee, September 9 through September 29, 1994-Continued

\begin{tabular}{|c|c|c|c|c|c|c|c|c|}
\hline $\begin{array}{c}\text { Site } \\
\text { number }\end{array}$ & $\begin{array}{l}\text { Type of } \\
\text { site }\end{array}$ & $\begin{array}{c}\text { Date } \\
\text { sampled } \\
\text { (month/ } \\
\text { day/year) }\end{array}$ & Method & $\begin{array}{l}\text { Flow } \\
\left(\mathrm{ft}^{3} / \mathrm{s}\right)\end{array}$ & pH & $\begin{array}{c}\text { Specific } \\
\text { conduc- } \\
\text { tance } \\
(\mu \mathrm{S} / \mathrm{cm})\end{array}$ & $\begin{array}{c}\text { Temper- } \\
\text { ature } \\
\left({ }^{\circ} \mathrm{C}\right)\end{array}$ & $\begin{array}{c}\text { Dis- } \\
\text { solved } \\
\text { oxygen } \\
\text { (mg/L) }\end{array}$ \\
\hline 16010 & Stream & $9 / 15 / 94$ & $\mathrm{E}$ & .01 & 8 & 316 & 18.5 & -- \\
\hline 16015 & Seep & $9 / 15 / 94$ & $\bar{D}$ & 0 & - & -- & - & -- \\
\hline 16020 & Seep & $9 / 15 / 94$ & D & 0 & -- & -- & -- & -- \\
\hline 16025 & Stream & $9 / 15 / 94$ & L & 0 & 7.7 & 198 & 19.0 & - \\
\hline 16030 & Seep & $9 / 15 / 94$ & D & 0 & - & -- & -- & -- \\
\hline 16035 & Stream & $9 / 15 / 94$ & D & 0 & -- & -- & -- & -- \\
\hline 16040 & Seep & $9 / 15 / 94$ & D & 0 & -- & -- & -- & - \\
\hline 16045 & Stream & $9 / 15 / 94$ & $\mathrm{D}$ & 0 & - & - & -- & - \\
\hline 16050 & Stream & $9 / 15 / 94$ & D & 0 & - & - & -- & -- \\
\hline 16055 & Spring & $9 / 15 / 94$ & $\mathrm{~L}$ & 0 & 7.7 & 91 & 20.0 & 1 \\
\hline 16060 & Seep & $9 / 15 / 94$ & D & 0 & -- & -- & -- & -. \\
\hline 16065 & Stream & $9 / 15 / 94$ & $\mathrm{~L}$ & 0 & 7 & 120 & 20.5 & -- \\
\hline 16067 & Seep & $9 / 15 / 94$ & D & 0 & -- & -- & - & $\therefore$ \\
\hline 16070 & Seep & $9 / 15 / 94$ & D & 0 & -- & -- & -- & -- \\
\hline 16075 & Stream & $9 / 15 / 94$ & D & 0 & -- & -- & -- & - \\
\hline 16080 & Seep & $9 / 15 / 94$ & D & 0 & -- & -- & -- & -- \\
\hline 16085 & Stream & $9 / 15 / 94$ & D & 0 & -- & -- & -- & -- \\
\hline 16090 & Stream & $9 / 15 / 94$ & D & 0 & -- & - & -- & -- \\
\hline 16095 & Seep & $9 / 15 / 94$ & D & 0 & -- & $-\cdot$ & -- & -- \\
\hline 16100 & Seep & $9 / 15 / 94$ & D & 0 & -- & -- & -- & -- \\
\hline 16105 & Stream & $9 / 15 / 94$ & L & 0 & 7.2 & 115 & 17.5 & -- \\
\hline 16110 & Seep & $9 / 15 / 94$ & D & 0 & -- & -- & $\cdot-$ & -- \\
\hline 17005 & Stream & $9 / 15 / 94$ & D & 0 & -- & -- & -- & - \\
\hline 17010 & Stream & $9 / 15 / 94$ & D & 0 & - & - & -- & -- \\
\hline 17015 & Stream & $9 / 15 / 94$ & D & 0 & -- & $-\cdot$ & -- & -- \\
\hline 17020 & Stream & $9 / 15 / 94$ & D & 0 & - & - & -- & -- \\
\hline 17030 & Stream & $9 / 15 / 94$ & D & 0 & -- & -- & -- & -- \\
\hline 17035 & Seep & $9 / 15 / 94$ & D & 0 & -- & -- & -- & -- \\
\hline 17040 & Stream & $9 / 15 / 94$ & D & 0 & -- & -- & -- & -- \\
\hline 17045 & Seep & $9 / 15 / 94$ & D & 0 & -- & -- & - & - \\
\hline 17050 & Stream & $9 / 15 / 94$ & D & 0 & -- & -- & -- & - \\
\hline 17055 & Seep & $9 / 15 / 94$ & D & 0 & - & - & -- & - \\
\hline 17065 & Seep & $9 / 16 / 94$ & D & 0 & - & -- & -- & $\cdots$ \\
\hline 17070 & Seep & $9 / 16 / 94$ & D & 0 & -- & -- & -- & -- \\
\hline 17075 & Stream & $9 / 16 / 94$ & D & 0 & -- & -- & -- & -- \\
\hline 17082 & Seep & $9 / 16 / 94$ & D & 0 & -- & -- & -- & -- \\
\hline 17083 & Seep & $9 / 16 / 94$ & D & 0 & -- & -- & -- & $-\cdot$ \\
\hline 17085 & Stream & $9 / 16 / 94$ & D & 0 & -- & -- & -- & - \\
\hline 17090 & Seep & 9/16/94 & D & 0 & -- & -- & - & -- \\
\hline 17095 & Stream & $9 / 16 / 94$ & D & 0 & -- & -- & -- & - \\
\hline 17100 & Seep & $9 / 16 / 94$ & D & 0 & $-\bar{z}$ & $\bar{\varepsilon}$ & $\overline{-}$ & -- \\
\hline 17105 & Stream & $9 / 16 / 94$ & $\mathbf{L}$ & 0 & 7.5 & 115 & 19.5 & - \\
\hline 17110 & Stream & $9 / 16 / 94$ & $\mathbf{L}$ & 0 & 7 & 262 & 19.0 & -- \\
\hline 17115 & Seep & $9 / 16 / 94$ & D & 0 & - & - & -- & -- \\
\hline 17120 & Seep & $9 / 16 / 94$ & D & 0 & -- & $=$ & -- & -- \\
\hline 17125 & Stream & $9 / 16 / 94$ & $\mathbf{E}$ & .01 & 7.7 & 92 & 19.5 & - \\
\hline 17130 & Seep & $9 / 16 / 94$ & $\mathbf{E}$ & .01 & 7.3 & 111 & 18.5 & 8.5 \\
\hline 17135 & Seep & $9 / 16 / 94$ & D & 0 & -- & -- & -- & -- \\
\hline 18005 & Stream & $9 / 14 / 94$ & D & 0 & - & -- & -- & -- \\
\hline 18010 & Seep & $9 / 14 / 94$ & D & 0 & -- & -- & - & -- \\
\hline
\end{tabular}


Table 5. Discharge and water-quality data for the low base flow seepage investigation at Bear Creek Valley, Oak Ridge, Tennessee, September 9 through September 29, 1994-Continued

\begin{tabular}{|c|c|c|c|c|c|c|c|c|}
\hline $\begin{array}{c}\text { Site } \\
\text { number }\end{array}$ & $\begin{array}{l}\text { Type of } \\
\text { site }\end{array}$ & $\begin{array}{c}\text { Date } \\
\text { sampled } \\
\text { (month/ } \\
\text { day/year) }\end{array}$ & Method & $\begin{array}{l}\text { Flow } \\
\left(\mathrm{tt}^{3} / \mathrm{s}\right)\end{array}$ & pH & $\begin{array}{c}\text { Specific } \\
\text { conduc- } \\
\text { tance } \\
(\mu \mathrm{S} / \mathrm{cm})\end{array}$ & $\begin{array}{l}\text { Temper- } \\
\text { ature } \\
\left({ }^{\circ} \mathrm{C}\right)\end{array}$ & $\begin{array}{c}\text { Dis- } \\
\text { solved } \\
\text { oxygen } \\
\text { (mghl) }\end{array}$ \\
\hline 18015 & Seep & $9 / 14 / 94$ & $\mathrm{D}$ & 0 & -- & -- & -- & -. \\
\hline 18020 & Stream & $9 / 14 / 94$ & $\mathbf{L}$ & 0 & 7.4 & 260 & 15.0 & -- \\
\hline 18025 & Seep & $9 / 15 / 94$ & $\bar{D}$ & 0 & - & -. &.- & -- \\
\hline 18060 & Stream & $9 / 14 / 94$ & $\mathbf{E}$ & .02 & 8.1 & 271 & 16.5 & -. \\
\hline 18065 & Seep & $9 / 14 / 94$ & $\mathrm{D}$ & 0 & -- & - & - & -- \\
\hline 18070 & Seep & $9 / 14 / 94$ & $\mathrm{D}$ & 0 & -- & -- & - & -- \\
\hline 18075 & Seep & $9 / 14 / 94$ & $\mathrm{D}$ & 0 & -- & -- & - & -- \\
\hline 18080 & Seep & $9 / 14 / 94$ & $\mathrm{D}$ & 0 & -. & -- & -- & -- \\
\hline 18085 & Spring & $9 / 14 / 94$ & $\vec{E}$ & .02 & 7.7 & 271 & 14.5 & 8.6 \\
\hline 18090 & Seep & $9 / 14 / 94$ & $\overline{\mathrm{D}}$ & 0 & -- & - & - & -- \\
\hline 18095 & Seep & $9 / 14 / 94$ & D & 0 & $\ldots$ & -- & -- & -- \\
\hline 18100 & Stream & $9 / 14 / 94$ & D & 0 & -- & -- & -- & - \\
\hline 18105 & Stream & $9 / 14 / 94$ & D & 0 & -- & - & -- & -- \\
\hline 18110 & Stream & $9 / 14 / 94$ & $\mathrm{~L}$ & 0 & 7.2 & 313 & 19.0 & -- \\
\hline 18115 & Seep & $9 / 14 / 94$ & D & 0 & -- & - & -- & -- \\
\hline 18120 & Seep & $9 / 14 / 94$ & D & 0 & -- & -- & -- & - \\
\hline 18125 & Seep & $9 / 14 / 94$ & D & 0 & -- & -- & -- & -- \\
\hline 18130 & Stream & $9 / 14 / 94$ & L & 0 & 7 & 190 & 18.5 & -- \\
\hline 18140 & Seep & $9 / 14 / 94$ & D & 0 & - & -- & - & $-\bar{a}$ \\
\hline 18145 & Seep & $9 / 14 / 94$ & $\mathbf{L}$ & 0 & 7 & 512 & 19.0 & 2.8 \\
\hline 18150 & Stream & $9 / 14 / 94$ & $\mathbf{E}$ & .01 & 7.6 & 168 & 19.5 & -- \\
\hline 18155 & Seep & $9 / 15 / 94$ & D & 0 & -- & -- & -- & -- \\
\hline 18160 & Stream & $9 / 15 / 94$ & $E$ & .01 & 7.8 & 142 & 18.5 & -- \\
\hline 18165 & Stream & $9 / 15 / 94$ & D & 0 & - & -- & -- & $\cdots$ \\
\hline 18170 & Stream & $9 / 15 / 94$ & $D$ & 0 & -- & - & -- & - \\
\hline 18175 & Seep & $9 / 15 / 94$ & D & 0 & -- & $\cdots$ & - & - \\
\hline 18180 & Seep & $9 / 15 / 94$ & $\bar{D}$ & 0 & -- & - & - & -- \\
\hline 18185 & Stream & $9 / 15 / 94$ & $\mathrm{E}$ & .01 & 7.7 & 153 & 18.5 & -- \\
\hline 18190 & Seep & $9 / 15 / 94$ & D & 0 & - & $=$ & $\overline{0}$ & - \\
\hline 18195 & Stream & $-9 / 15 / 94$ & $E$ & .01 & 7.7 & 155 & 18.5 & - \\
\hline 18215 & Seep & $9 / 15 / 94$ & $\mathrm{E}$ & .01 & 7.5 & 158 & 17.5 & -- \\
\hline 18220 & Seep & $9 / 15 / 94$ & $\overline{\mathbf{L}}$ & 0 & 7.6 & 142 & 18.5 & - \\
\hline 18225 & Seep & $9 / 15 / 94$ & $D$ & 0 & -- & -- & -- & - \\
\hline 18230 & Seep & $9 / 15 / 94$ & D & 0 & - & -- & - & - \\
\hline 18250 & Stream & $9 / 15 / 94$ & D & 0 & - & - & - & - \\
\hline 18255 & Seep & $9 / 15 / 94$ & D & 0 & -- & -- & - & -- \\
\hline 18260 & Stream & $9 / 15 / 94$ & D & 0 & - & -- & - & -- \\
\hline 19003 & Spring & $9 / 16 / 94$ & D & 0 & -- & -- & $\cdots$ & - \\
\hline 19004 & Stream & $9 / 16 / 94$ & - D & 0 & -- & -- & - & - \\
\hline 19005 & Seep & $9 / 16 / 94$ & D & 0 & - & - & - & -- \\
\hline 19007 & Spring & $9 / 16 / 94$ & D & 0 & -- & - & - & $\cdots$ \\
\hline 19008 & Stream & $9 / 16 / 94$ & $\mathbf{E}$ & .02 & 8.2 & 250 & - & -- \\
\hline 19010 & Seep & $9 / 16 / 94$ & D & 0 & $\bar{z}$ & $\bar{n}$ & $\overline{10}$ & -- \\
\hline 19015 & Stream & $9 / 16 / 94$ & $\bar{E}$ & .01 & 7.9 & 262 & 19.5 & -- \\
\hline 19045 & Stream & $9 / 16 / 94$ & $\overline{\mathbf{D}}$ & 0 & - & $\overline{-}$ & $\overline{-}$ & -- \\
\hline 19050 & Stream & $9 / 16 / 94$ & $\mathbf{L}$ & 0 & 7.7 & 270 & 19.5 & - \\
\hline 19055 & Seep & $9 / 16 / 94$ & D & 0 & -- & -- & -- & -- \\
\hline 19090 & Seep & $9 / 16 / 94$ & D & 0 & $\overline{70}$ & $\overline{5}$ & $\ddot{11}$ & -- \\
\hline 19095 & Stream & $9 / 16 / 94$ & $\mathbf{P}$ & .02 & 7.9 & 235 & 21 & -- \\
\hline 19100 & Stream & $9 / 16 / 94$ & D & 0 & -- & -- & - & - \\
\hline
\end{tabular}


Table 5. Discharge and water-quality data for the low base flow seepage investigation at Bear Creek Valley, Oak Ridge, Tennessee, September 9 through September 29, 1994-Continued

\begin{tabular}{|c|c|c|c|c|c|c|c|c|}
\hline $\begin{array}{c}\text { Site } \\
\text { number }\end{array}$ & $\begin{array}{l}\text { Type of } \\
\text { site }\end{array}$ & $\begin{array}{c}\text { Date } \\
\text { sampled } \\
\text { (month/ } \\
\text { day/year) }\end{array}$ & Method & $\begin{array}{l}\text { Flow } \\
\left(f t^{3} / s\right)\end{array}$ & $\mathrm{pH}$ & $\begin{array}{c}\text { Specific } \\
\text { conduc- } \\
\text { tance } \\
(\mu \mathrm{S} / \mathrm{cm})\end{array}$ & $\begin{array}{c}\text { Temper- } \\
\text { ature } \\
\left.{ }^{\circ} \mathrm{C}\right)\end{array}$ & $\begin{array}{c}\text { Dis- } \\
\text { solved } \\
\text { oxygen } \\
\text { (mg/L) } \\
\end{array}$ \\
\hline 19105 & Stream & $9 / 16 / 94$ & D & 0 & -. & -- & -- & -- \\
\hline 19110 & Seep & $9 / 16 / 94$ & D & 0 & - & -- & -. & -- \\
\hline 19115 & Stream & $9 / 16 / 94$ & $\bar{L}$ & 0 & 7.7 & 192 & 18.0 & -- \\
\hline 19120 & Seep & $9 / 16 / 94$ & $\bar{D}$ & 0 & -- & -. & -- & -- \\
\hline 19125 & Wetland & $9 / 16 / 94$ & D & 0 & -- & -- & -- & -- \\
\hline 19130 & Seep & $9 / 15 / 94$ & D & 0 & -- & -- & -- & .- \\
\hline 19135 & Stream & $9 / 16 / 94$ & $\bar{E}$ & .01 & 7.6 & 216 & 18.5 & -- \\
\hline 19140 & Stream & $9 / 16 / 94$ & $\overline{\mathrm{D}}$ & 0 & -- & -- & -- & - \\
\hline 19145 & Stream & $9 / 16 / 94$ & $\mathrm{D}$ & 0 & -- & -- & -- & -- \\
\hline 19146 & Stream & $9 / 16 / 94$ & $\mathrm{D}$ & 0 & -- & -- & -- & - \\
\hline 19148 & Seep & $9 / 16 / 94$ & D & 0 & -- & -- & -- & - \\
\hline 19150 & Seep & $9 / 16 / 94$ & D & 0 & -- & -- & -- & - \\
\hline 19155 & Stream & $9 / 16 / 94$ & D & 0 & -- & -- & -- & - \\
\hline 19156 & Stream & $9 / 16 / 94$ & D & 0 & -. & -- & -- & -- \\
\hline 19158 & Seep & $9 / 16 / 94$ & $\mathrm{D}$ & 0 & - & -- & -- & -. \\
\hline 19160 & Stream & $9 / 16 / 94$ & D & 0 & - & -- & -- & -- \\
\hline 19165 & Seep & $9 / 16 / 94$ & D & 0 & -- & -. & -- & -- \\
\hline 19170 & Seep & $9 / 16 / 94$ & D & 0 & -. & -- & -. & -. \\
\hline 19175 & Seep & $9 / 16 / 94$ & D & 0 & -. & -- & $\ddot{n}$ & -- \\
\hline 19180 & Stream & $9 / 16 / 94$ & L & 0 & 8 & 218 & 17.5 & -- \\
\hline 19285 & Stream & $9 / 16 / 94$ & D & 0 & -- & -- & -- & -- \\
\hline 19290 & Stream & $9 / 16 / 94$ & D & 0 & - & - & -- & $\cdot-$ \\
\hline 19295 & Seep & $9 / 16 / 94$ & D & 0 & -- & -- & -- & -- \\
\hline 19300 & Stream & $9 / 16 / 94$ & E & .01 & - & -- & - & - \\
\hline 20005 & Stream & $9 / 16 / 94$ & $\overline{\mathbf{P}}$ & .17 & 8.1 & 329 & 18.5 & -. \\
\hline 20010 & Stream & $9 / 16 / 94$ & $\mathrm{P}$ & -- & 7.6 & 385 & 19.5 & - \\
\hline 20015 & Stream & $9 / 15 / 94$ & L & 0 & 7.3 & 301 & 19.0 & -- \\
\hline 20020 & Seep & $9 / 15 / 94$ & $\bar{D}$ & 0 & -- & - & $-\overline{-}$ & -- \\
\hline 20250 & Stream & $9 / 16 / 94$ & $\mathbf{P}$ & .05 & 8 & 322 & 18.5 & -- \\
\hline 20255 & Stream & $9 / 16 / 94$ & $\mathbf{P}$ & .12 & 8 & 325 & 17.5 & - \\
\hline 20258 & Seep & $9 / 16 / 94$ & D & 0 & .. & -- & - & - \\
\hline 20260 & Stream & 9/16/94 & $\mathbf{P}$ & .13 & 8.1 & 326 & 17.0 & -- \\
\hline 20265 & Stream & $9 / 15 / 94$ & $\mathbf{P}$ & $=$ & 6.5 & 180 & 19.5 & -- \\
\hline 20280 & Stream & $9 / 15 / 94$ & L & 0 & 7 & 424 & 18.0 & -- \\
\hline 20285 & Seep & $9 / 15 / 94$ & $\bar{D}$ & 0 & -- & -- & -- & -- \\
\hline 20290 & Stream & 9/15/94 & D & 0 & -. & -- & -- & -- \\
\hline 20295 & Seep & $9 / 15 / 94$ & D & 0 & -- & -- & -- & -- \\
\hline 20300 & Seep & $9 / 15 / 94$ & D & 0 &.- & -- & -- & -- \\
\hline 20305 & Stream & $9 / 15 / 94$ & D & 0 & -- & -- & - & -- \\
\hline 20310 & Seep & $9 / 15 / 94$ & D & 0 & - & $-\cdot$ & -- & -- \\
\hline 20315 & Seep & $9 / 15 / 94$ & $\mathrm{~L}$ & 0 & 7.5 & 132 & 19.5 & 7.7 \\
\hline $\begin{array}{l}20320 \\
20325\end{array}$ & Seep & $\begin{array}{l}9 / 15 / 94 \\
9 / 15 / 94\end{array}$ & D & $\begin{array}{l}0 \\
0\end{array}$ & 7.7 & 121 & 19.0 & $\ldots$ \\
\hline $\begin{array}{l}20325 \\
20335\end{array}$ & $\begin{array}{l}\text { Stream } \\
\text { Seep }\end{array}$ & $\begin{array}{l}9 / 15 / 94 \\
9 / 15 / 94\end{array}$ & L & 0 & 7.3 & 125 & 17.5 & 7.5 \\
\hline 20340 & Stream & - & $=$ & - & -- & -- & -- & -- \\
\hline 20342 & Stream & $9 / 15 / 94$ & D & 0 & - & - & - & -- \\
\hline 20343 & Stream & $9 / 15 / 94$ & D & 0 & $\because$ & - & $\overline{10}=$ & -- \\
\hline 20350 & Ștream & $9 / 15 / 94$ & E & .01 & 7.5 & 344 & 18.5 & - \\
\hline 20355 & Seep & $9 / 15 / 94$ & L & 0 & 7 & 238 & 21.0 & -- \\
\hline 20360 & Stream & $9 / 15 / 94$ & L & 0 & 7.1 & 134 & 18.0 & -- \\
\hline
\end{tabular}


Table 5. Discharge and water-quality data for the low base flow seepage investigation at Bear Creek Valley, Oak Ridge, Tennessee, September 9 through September 29, 1994-Continued

\begin{tabular}{|c|c|c|c|c|c|c|c|c|}
\hline $\begin{array}{c}\text { Site } \\
\text { number }\end{array}$ & $\begin{array}{l}\text { Type of } \\
\text { site }\end{array}$ & $\begin{array}{c}\text { Date } \\
\text { sampled } \\
\text { (month/ } \\
\text { day/year) }\end{array}$ & Method & $\begin{array}{l}\text { Flow } \\
\left(\mathrm{ft}^{3} / \mathrm{s}\right)\end{array}$ & pH & $\begin{array}{c}\text { Specific } \\
\text { conduc- } \\
\text { tance } \\
(\mu \mathrm{S} / \mathrm{cm})\end{array}$ & $\begin{array}{c}\text { Temper- } \\
\text { ature } \\
\left({ }^{\circ} \mathrm{C}\right)\end{array}$ & $\begin{array}{c}\text { Dis- } \\
\text { solved } \\
\text { oxygen } \\
\text { (mg/L) }\end{array}$ \\
\hline $\begin{array}{l}20365 \\
20370 \\
20375 \\
20380 \\
20385 \\
20390 \\
20395 \\
20400 \\
20405 \\
20410\end{array}$ & $\begin{array}{l}\text { Stream } \\
\text { Stream } \\
\text { Seep } \\
\text { Seep } \\
\text { Seep } \\
\text { Stream } \\
\text { Seep } \\
\text { Seep } \\
\text { Seep } \\
\text { Stream }\end{array}$ & $\begin{array}{l}9 / 15 / 94 \\
9 / 15 / 94 \\
9 / 15 / 94 \\
9 / 15 / 94 \\
9 / 15 / 94 \\
9 / 15 / 94 \\
9 / 15 / 94 \\
9 / 15 / 94 \\
9 / 15 / 94 \\
9 / 15 / 94\end{array}$ & $\begin{array}{l}\text { L } \\
D \\
D \\
D \\
D \\
D \\
D \\
\text { L } \\
D \\
\text { L }\end{array}$ & $\begin{array}{l}0 \\
0 \\
0 \\
0 \\
0 \\
0 \\
0 \\
0 \\
0 \\
0\end{array}$ & $\begin{array}{l}6.1 \\
- \\
- \\
- \\
- \\
- \\
-\overline{6} \\
6.7 \\
\overline{7} .5\end{array}$ & $\begin{array}{r}226 \\
-- \\
-- \\
- \\
- \\
-- \\
-- \\
227 \\
-- \\
73\end{array}$ & $\begin{array}{c}18.5 \\
-- \\
-- \\
- \\
-- \\
-- \\
-- \\
18.5 \\
- \\
18.5\end{array}$ & $\begin{array}{l}-- \\
- \\
-- \\
-- \\
- \\
-- \\
-- \\
4.2 \\
-- \\
--\end{array}$ \\
\hline $\begin{array}{l}20415 \\
20420 \\
20425 \\
20430 \\
21005 \\
21010 \\
21015 \\
21020 \\
21025 \\
21030\end{array}$ & $\begin{array}{l}\text { Seep } \\
\text { Stream } \\
\text { Stream } \\
\text { Stream } \\
\text { Stream } \\
\text { Seep } \\
\text { Stream } \\
\text { Spring } \\
\text { Stream } \\
\text { Spring }\end{array}$ & $\begin{array}{l}9 / 15 / 94 \\
9 / 15 / 94 \\
9 / 15 / 94 \\
9 / 15 / 94 \\
9 / 15 / 94 \\
9 / 15 / 94 \\
9 / 15 / 94 \\
9 / 15 / 94 \\
9 / 15 / 94 \\
9 / 15 / 94\end{array}$ & $\begin{array}{l}\text { L } \\
\text { D } \\
\text { D } \\
\text { P } \\
\text { L } \\
\text { D } \\
\text { P } \\
\text { L } \\
\text { D } \\
\text { D }\end{array}$ & $\begin{array}{l}0 \\
0 \\
0 \\
.15 \\
0 \\
0 \\
.03 \\
0 \\
0 \\
0\end{array}$ & $\begin{array}{l}7.2 \\
-- \\
\ddot{7.9} \\
6.4 \\
\ddot{7} \\
6.9 \\
-- \\
--\end{array}$ & $\begin{array}{r}78 \\
-- \\
-- \\
330 \\
177 \\
-- \\
302 \\
291 \\
- \\
--\end{array}$ & $\begin{array}{l}17.0 \\
-- \\
- \\
16.0 \\
21.5 \\
-\overline{19.5} \\
19.5 \\
-- \\
--\end{array}$ & $\begin{array}{l}8.7 \\
- \\
- \\
-- \\
-- \\
-- \\
\overline{7} \\
-- \\
--\end{array}$ \\
\hline $\begin{array}{l}21035 \\
21040 \\
21045 \\
21050 \\
21055 \\
21057 \\
21058 \\
21060 \\
21065 \\
21100\end{array}$ & $\begin{array}{l}\text { Stream } \\
\text { Seep } \\
\text { Stream } \\
\text { Seep } \\
\text { Stream } \\
\text { Stream } \\
\text { Seep } \\
\text { Spring } \\
\text { Stream } \\
\text { Stream }\end{array}$ & $\begin{array}{l}9 / 15 / 94 \\
9 / 15 / 94 \\
9 / 15 / 94 \\
9 / 15 / 94 \\
9 / 15 / 94 \\
9 / 15 / 94 \\
9 / 15 / 94 \\
9 / 15 / 94 \\
9 / 15 / 94 \\
9 / 15 / 94\end{array}$ & $\begin{array}{l}\text { L } \\
\text { D } \\
\text { D } \\
\text { L } \\
\text { V } \\
\text { L } \\
\text { D } \\
\text { L } \\
\text { D } \\
\text { P }\end{array}$ & $\begin{array}{l}0 \\
0 \\
0 \\
0 \\
.04 \\
0 \\
0 \\
0 \\
0 \\
.02\end{array}$ & $\begin{array}{l}7.2 \\
-- \\
-7.2 \\
7.2 \\
7.2 \\
6.2 \\
-- \\
6.5 \\
-\overline{7.3}\end{array}$ & $\begin{array}{r}127 \\
-- \\
- \\
55 \\
65 \\
87 \\
-- \\
26 \\
-\overline{155}\end{array}$ & $\begin{array}{l}18.5 \\
-- \\
-- \\
18.5 \\
19.0 \\
18.5 \\
-\overline{17.5} \\
\overline{22.0}\end{array}$ & \begin{tabular}{l}
-- \\
-- \\
\hdashline 8.1 \\
-- \\
-- \\
-- \\
7.8 \\
-- \\
--
\end{tabular} \\
\hline $\begin{array}{l}21102 \\
21103 \\
21105 \\
21110 \\
21115 \\
21119 \\
21120 \\
21125 \\
21130 \\
22005\end{array}$ & $\begin{array}{l}\text { Stream } \\
\text { Seep } \\
\text { Stream } \\
\text { Seep } \\
\text { Seep } \\
\text { Seep } \\
\text { Stream } \\
\text { Stream } \\
\text { Seep } \\
\text { Stream }\end{array}$ & $\begin{array}{l}9 / 15 / 94 \\
9 / 15 / 94 \\
9 / 15 / 94 \\
9 / 15 / 94 \\
9 / 15 / 94 \\
9 / 15 / 94 \\
9 / 15 / 94 \\
9 / 15 / 94 \\
9 / 15 / 94 \\
9 / 16 / 94\end{array}$ & $\begin{array}{l}D \\
D \\
P \\
D \\
\text { V } \\
D \\
\text { V } \\
D \\
D \\
P\end{array}$ & 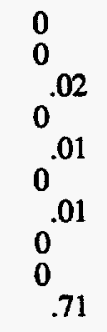 & $\begin{array}{l}-- \\
\overline{7.3} \\
\overline{7} .5 \\
\overline{7.3} \\
-- \\
\overline{7} \\
\overline{7}\end{array}$ & $\begin{array}{r}-- \\
152 \\
\ddot{-} \\
147 \\
-\ddot{1} \\
184 \\
-- \\
-- \\
239\end{array}$ & $\begin{array}{l}-\overline{20.0} \\
\overline{20.0} \\
\overline{19.5} \\
-- \\
\overline{19.5}\end{array}$ & $\begin{array}{l}-- \\
-- \\
- \\
- \\
8.4 \\
-- \\
-- \\
-- \\
--\end{array}$ \\
\hline $\begin{array}{l}22006 \\
22010 \\
22034 \\
22040 \\
22041 \\
22042 \\
22044 \\
22045 \\
22050 \\
22055 \\
22060\end{array}$ & $\begin{array}{l}\text { Seep } \\
\text { Stream } \\
\text { Seep } \\
\text { Stream } \\
\text { Seep } \\
\text { Stream } \\
\text { Stream } \\
\text { Stream } \\
\text { Stream } \\
\text { Stream } \\
\text { Stream }\end{array}$ & $\begin{array}{l}9 / 16 / 94 \\
9 / 16 / 94 \\
9 / 16 / 94 \\
9 / 16 / 94 \\
9 / 16 / 94 \\
9 / 16 / 94 \\
9 / 16 / 94 \\
9 / 16 / 94 \\
9 / 16 / 94 \\
9 / 16 / 94 \\
9 / 16 / 94\end{array}$ & $\begin{array}{l}D \\
D \\
D \\
P \\
D \\
D \\
D \\
D \\
L \\
L \\
P\end{array}$ & $\begin{array}{l}0 \\
0 \\
0 \\
0 \\
0 \\
0 \\
0 \\
0 \\
0 \\
0 \\
.02 \\
\end{array}$ & $\begin{array}{l}-- \\
-- \\
-\overline{7.8} \\
-- \\
-- \\
-- \\
- \\
7.8 \\
7.5 \\
8 \\
\end{array}$ & $\begin{array}{r}- \\
- \\
- \\
356 \\
- \\
- \\
-- \\
- \\
394 \\
556 \\
286 \\
\end{array}$ & $\begin{array}{l}-. \\
- \\
- \\
19.5 \\
- \\
-- \\
-- \\
- \\
20.5 \\
17.0 \\
20.5 \\
\end{array}$ & $\begin{array}{l}- \\
- \\
- \\
- \\
- \\
- \\
- \\
- \\
- \\
- \\
-\end{array}$ \\
\hline
\end{tabular}


NOTICE

Page(s) size did not permit electronic reproduction. Information may be purchased by the general public from the National Technical Information Service, U.S. Department of Commerce, Springfield, VA 22161 (Area Code 703-487-4650). DOE and DOE contractors may purchase information by contacting DOE's Office of Scientific and Technical Information, P.O. Box 62, Oak Ridge, TN 37831, Attn: Information Services (Area Code 423-576-8401). 